\title{
Synthesis of sulfonamide-bridged glycomimetics
}

Marie Lopez ${ }^{\dagger}$, Laurent F. Bornaghi ${ }^{\dagger}$, Hugues Driguez ${ }^{\S}$ and Sally-Ann Poulsen ${ }^{\dagger}{ }^{\dagger *}$

†Eskitis Institute, Griffith University, Nathan Campus, Queensland, 4111, Australia

${ }^{\S}$ Centre de Recherche sur les Macromolécules Végétales, UPR-CNRS 5301, BP53, 38041

Grenoble, France

Corresponding author: s.poulsen@griffith.edu.au 


\section{Table of Contents Graphic}

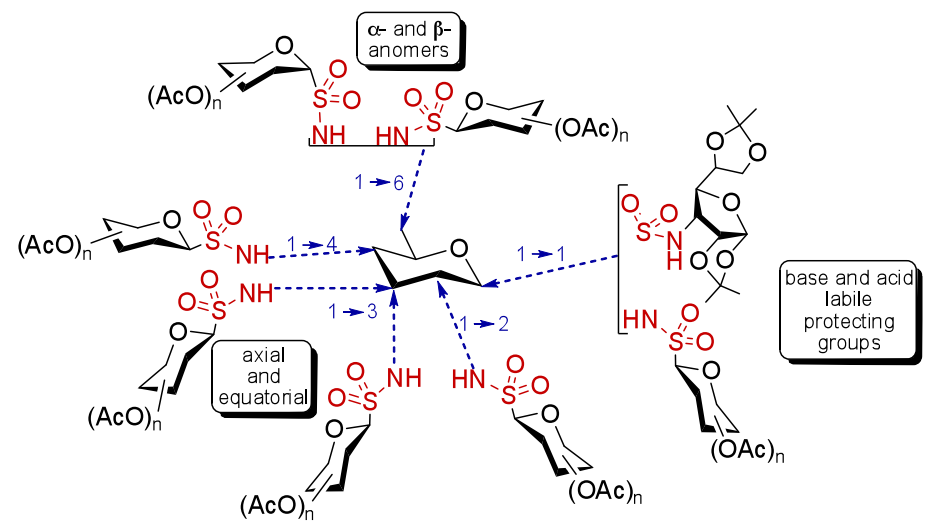




\begin{abstract}
A flexible and short synthesis of sulfonamide-bridged di-, tri-, tetra- and octasaccharide glycomimetics was accomplished by reaction of glycosyl thioacetates with amino sugar substrates. The chemistry to incorporate the sulfonamide linker in place of a native $O$-glycosidic bond was broadly scoped, allowing access to head-to-head $(1 \leftrightarrow 1)$ and head-to-tail $(1 \rightarrow 2),(1 \rightarrow 3),(1 \rightarrow 4)$ and $(1 \rightarrow 6)$ sulfonamide-bridged glycomimetics. The synthesis proceeds with retention of configuration at the anomeric centre, is compatible with variable stereochemical arrangements and with acid- and base-labile protecting groups.
\end{abstract}




\section{Introduction}

Carbohydrates play an important role in diverse biological events and in principle offer immense opportunities for therapeutic drug development, yet in practice constitute relatively few drugs. ${ }^{1}$ Native glycosidic bonds are sensitive to hydrolysis by endogenous glycoside hydrolases, and it is this liability that has led to the avoidance of carbohydrate-based molecules for in vivo applications. ${ }^{1}$ Synthetic glycomimetics are designed to circumvent in vivo problems and recent trends demonstrate that glycomimetics are increasingly emerging as lead compounds for drug discovery and as probes for chemical biology research. ${ }^{1,2}$ The rationale is to replace the enzymatically labile glycosidic bond with a covalent, robust, non-native linker to address inherent metabolic instability.

The development of straightforward synthetic methods towards novel, stable and short linkers between sugar building blocks is needed to support the discovery of new glycomimetics with potential bioactive properties. ${ }^{2}$ The structural complexity of natural carbohydrates stems from linking saccharide building blocks in variable arrangements via a glycosidic bond, hence the synthesis of both head-to-head and head-to-tail linkages is ideally sought. ${ }^{2}$ Existing replacements for the native glycosidic bond, comprising one or two bridging atoms between the pyranosyl moieties, are summarized in Figure 1. It is apparent that relatively few head-to-head $(1 \leftrightarrow 1)$ or headto-tail $(1 \rightarrow 2),(1 \rightarrow 3),(1 \rightarrow 4)$ and $(1 \rightarrow 6)$ linkers are known in the literature. Highly significant to this study is that straightforward and general synthetic methodology to link two sugars is not yet available. The $S$ - and $C$-glycosides are the most well documented replacements for the native glycosidic bond. ${ }^{3}$ 


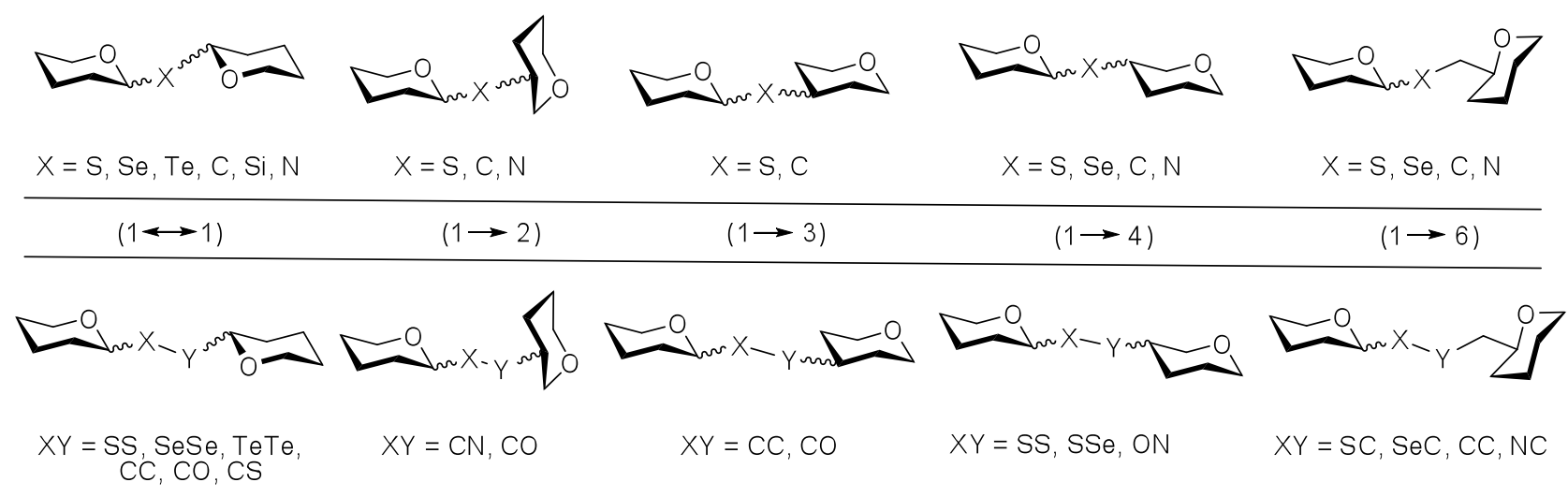

Figure 1. Known head-to-head $(1 \leftrightarrow 1)^{4-8}$ or head-to-tail $(1 \rightarrow 2)^{9-12},(1 \rightarrow 3)^{13,14},(1 \rightarrow 4)^{15-18}$ and $(1 \rightarrow 6)^{19-23}$ glycomimetic disaccharides. ( $\sim 0$ represents the pyranosyl moiety).

\section{Results and Discussion}

Given there is no prior general methodology that provides a common synthetic route to glycomimetics linked via the anomeric centre of one glycosyl residue to all positions of the second residue we decided to target this challenge. Our laboratory recently developed a synthesis of $S$ glycosyl primary sulfonamides, ${ }^{24,25}$ a class of molecules that had remained elusive using conventional synthetic routes towards sulfonamides. Herein we expand the utility of our recent synthetic methodology to covalently tether sugar moieties via a sulfonamide bridge. The sulfonamide moiety has not previously been utilized to join carbohydrates to generate either $S$ - or $N$-linked carbohydrates of the type $\left[(\text { sugar })_{n}-\mathrm{SO}_{2}-\mathrm{NH}-(\text { sugar })_{\mathrm{n}}, \mathrm{n} \geq 1\right]$. We first explored the synthesis of $(1 \leftrightarrow 1),(1 \rightarrow 2),(1 \rightarrow 3),(1 \rightarrow 4)$ and $(1 \rightarrow 6)$ sulfonamide-bridged disaccharides, Figure 2 .

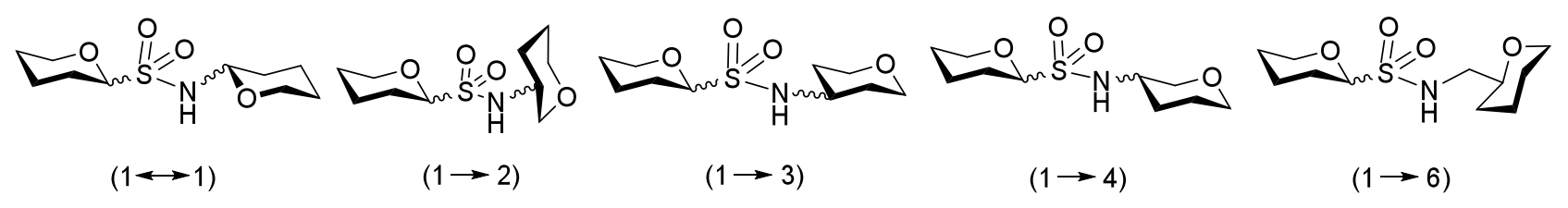

Figure 2. Target sulfonamide-bridged disaccharides. ( 
The chemistry we proposed had a requirement for $S$-glycosyl thioacetate and a panel of amino sugar partners as reagents. These carbohydrate reagents are readily available from either commercial sources and/or through chemical synthesis. ${ }^{26,27}$ Specifically, we examined the reaction of 1-S-acetyl-2,3,4,6-tetra- $O$-acetyl-1-thio- $\beta$-D-glucopyranose (1) with each of per- $O$-acetylated glucosylamine 6, 2-glucosamine (7), 3-amino-glucose (8), methyl 4-amino-glucoside (9) and methyl 6-amino-glucoside (10), Scheme 1. The reaction of thioacetate 1 with amines 6-10 in the presence of diethylbromomalonate generated the corresponding sulfenamide linked disaccharides in all cases (14a-18a). Oxidation of 14a-18a with excess $m$-CPBA gave the target sulfonamide-linked disaccharide mimetics $\mathbf{1 4 b}$-18b. The $O$-acetyl groups were removed using standard Zemplén conditions $^{30}$ to liberate the fully deprotected, water soluble sulfonamide-bridged disaccharides $\mathbf{1 4 c}$ 18c. The stereoselectivity of the reactions was evidenced from characteristic ${ }^{3} J_{1,2}$ coupling constants for each saccharide partner. 
SCHEME 1. Synthesis of $(1 \leftrightarrow 1),(1 \rightarrow 2),(1 \rightarrow 3),(1 \rightarrow 4)$ and $(1 \rightarrow 6)$ sulfonamide-bridged disaccharides.

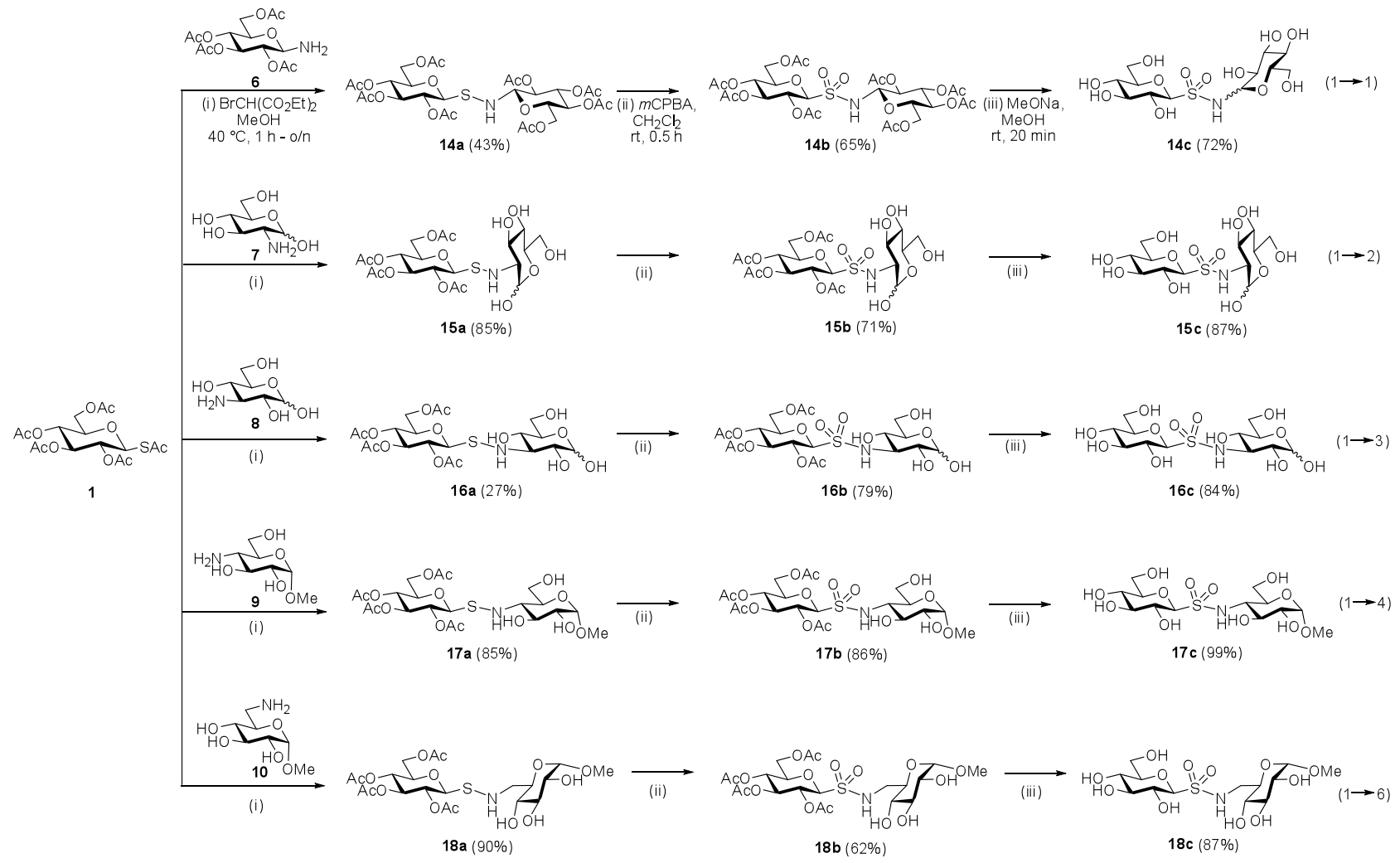

Having established that the sulfonamide moiety provides a general linker for glycomimetic disaccharides in just two straightforward synthetic steps, we were encouraged to extend our investigation to the reaction of larger thioacetate substrates, including the thioacetyl disaccharides $(2,3)$ and heptasaccharide $(4)$. The reaction of these thioacetates with monosaccharide amino sugars 7, 10 and the disaccharide per- $O$-acetylated maltosyl amine (11) proceeded to generate sulfonamide-bridged trisaccharides 19a-c, 20a-c and 21a-c, tetrasaccharides 22a-c and octasaccharides 23a-b, respectively, Table 1, entries 1-5. We next explored whether stereochemical characteristics of reagents, such as $\alpha-, \beta$-, equatorial or axial, had implications to the generality of 
this method. 1-S-Acetyl-2,3,4,6-tetra- $O$-acetyl-1-thio- $\alpha$-D-mannopyranose (5) and methyl 3-amino alloside (12) allowed the effect of an $\alpha$-anomer of the thioacetate and an axial amine to be assessed, respectively. There were no notable differences in reaction behaviour of 5 ( $\alpha$-anomer) and 1 ( $\beta$ anomer) or 12 (axial amine) compared to 8 (equatorial amine), Table 1, entries 6 and 7.
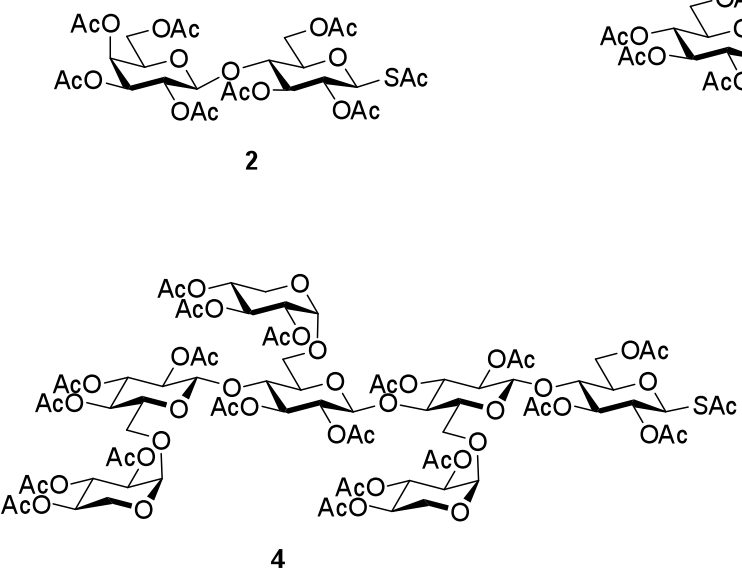
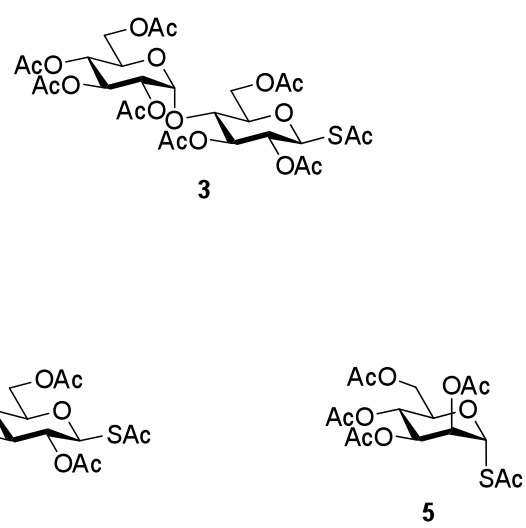

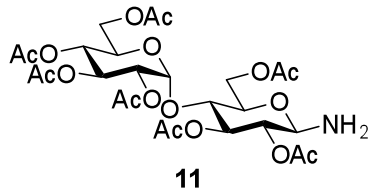

11

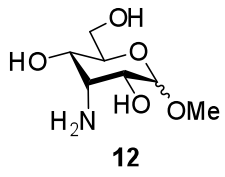


TABLE 1. Investigation of the scope of the sulfonamide moiety as a glycomimetic linker.

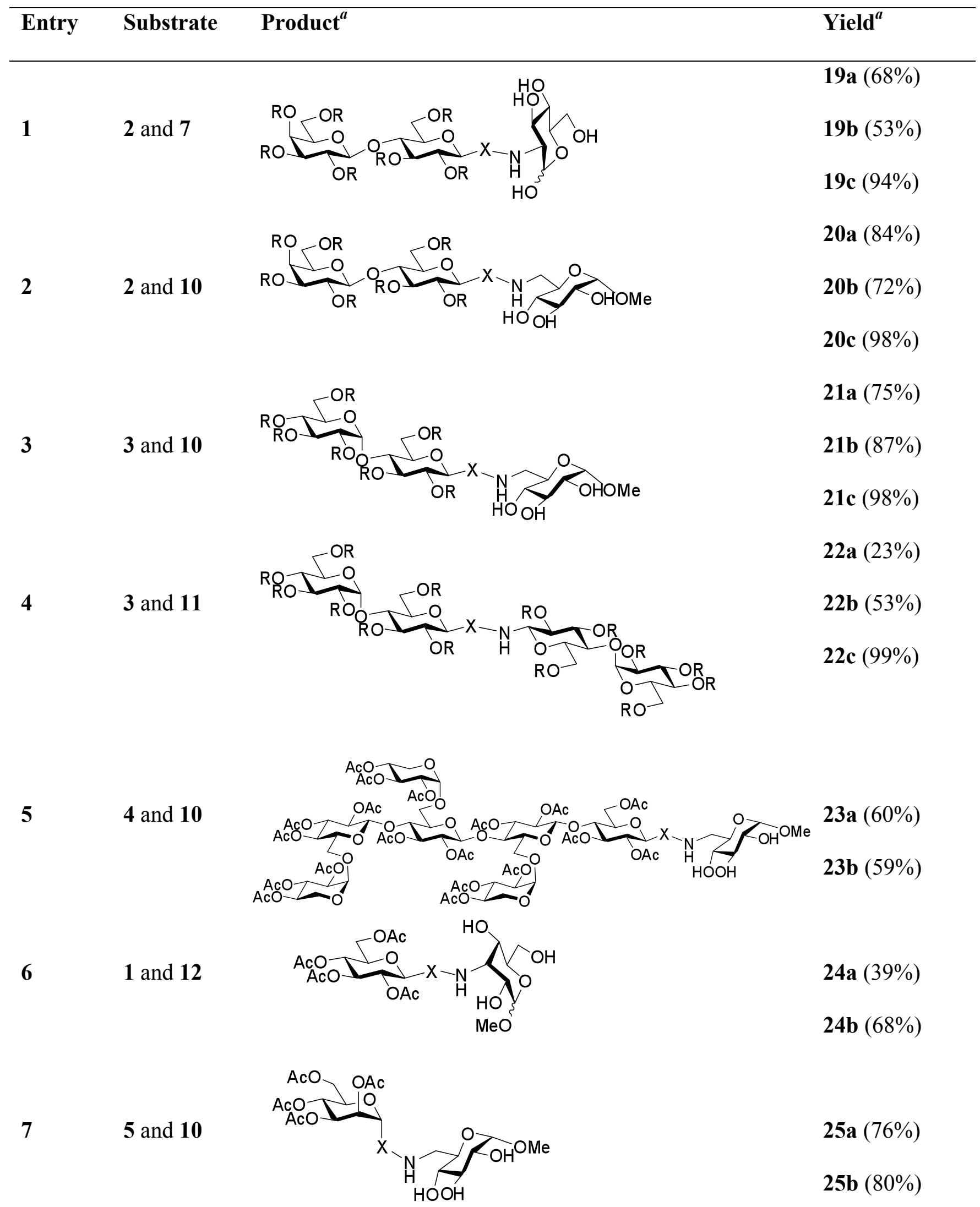


${ }^{a}$ a: $\mathrm{X}=\mathrm{S}, \mathrm{R}=\mathrm{Ac} ; \mathrm{b}: \mathrm{X}=\mathrm{SO}_{2}, \mathrm{R}=\mathrm{Ac} ; \mathrm{c}: \mathrm{X}=\mathrm{SO}_{2}, \mathrm{R}=\mathrm{H}$.

The yield of purified sulfenamides was in general good, however in cases where the yield was low this could be readily accounted for. The reaction to form sulfenamides $\mathbf{1 4 a}(43 \%)$ and $\mathbf{2 2 a}$ $(23 \%)$ proceeds with the formation of two side-products: (i) a disulfide ${ }^{29}$ and (ii) a diglycosylamine (possible only from amino sugars 6 and 11). ${ }^{30}$ The side-products were formed in small amounts however their chromatographic separation from the target sulfenamides proved difficult owing to similar physical properties. The lower yield for the synthesis of the $(1 \rightarrow 3)$ analog $\mathbf{1 6 a}(27 \%)$ and $\mathbf{2 4 a}(39 \%)$ may be a result of the possible lower reactivity of the 3-position of reagents 8 and $\mathbf{1 2} .^{31}$

As a further test of the scope of this methodology we assessed the presence and removal of base- and acid-labile protecting groups, a combination commonly encountered in carbohydrate chemistry. Amine 13, a furanose derivative synthesized from commercially available 1,2:5,6-di- $O$ isopropylidene- $\alpha$-D-glucofuranose, comprises two isopropylidene acetal protecting groups.

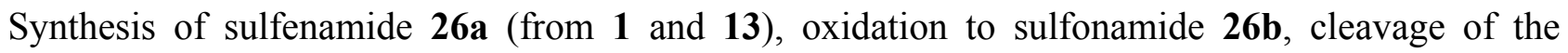
acetates of $\mathbf{2 6 b}$ to give $\mathbf{2 6 c}$, followed by stepwise removal of the acetals of $\mathbf{2 6 c}$ to give $\mathbf{2 6 \mathbf { d }}$ and $\mathbf{2 6 e}$, respectively, confirmed that the sulfonamide glycomimetics are stable to both the base and acid conditions required for the removal of the respective protecting groups, Scheme 2.

\section{SCHEME 2. Compatibility with base- and acid-labile protecting groups.}




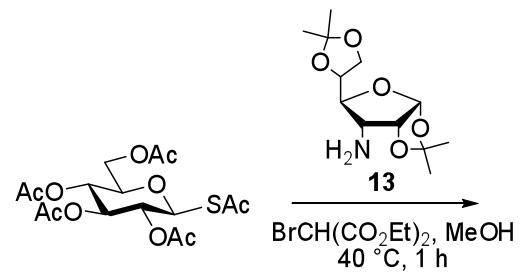

1

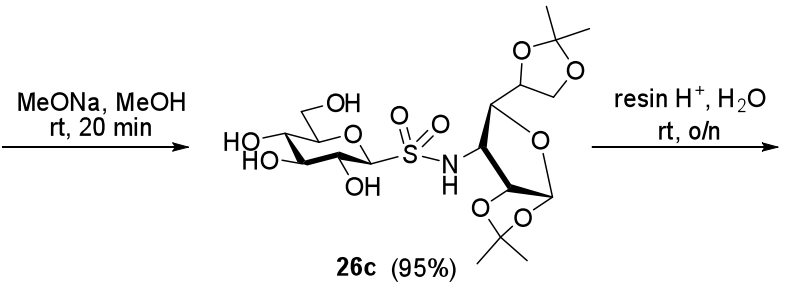

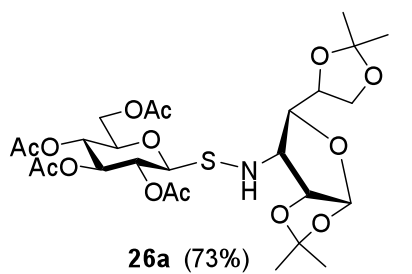

$m \mathrm{CPBA}, \mathrm{CH}_{2} \mathrm{Cl}_{2}$
$\mathrm{rt}, 0.5 \mathrm{~h}$

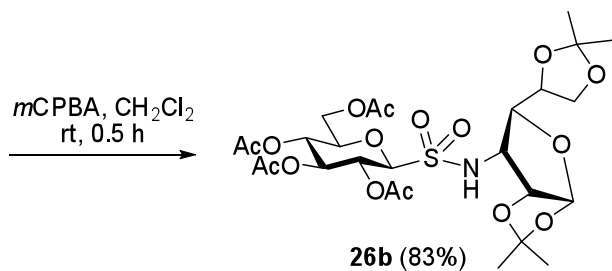

26 b (83\%)

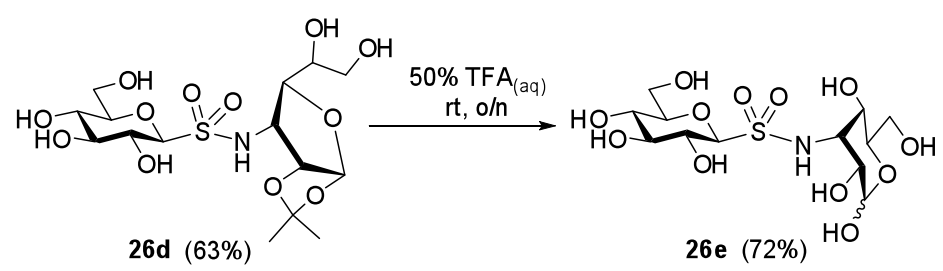

\section{Conclusion}

In summary, we have targeted the synthesis of glycomimetics, a challenging area of chemistry with a high and, until now, unmet demand for broadly scoped and general synthetic approaches towards robust glycomimetic linkers. We have synthesized sulfonamide-bridged di-, tri-, tetra- and octasaccharide glycomimetics using a uniform, straightforward and short synthesis starting from a selection of readily accessible 1-thioacetyl glycose and amino sugar reagents. The primary limitation identified with this approach is a requirement to use excess reagents. The incorporation of the sulfonamide linker in place of a native $O$-glycosidic bond has allowed access to head-to-head $(1 \leftrightarrow 1)$ and head-to-tail $(1 \rightarrow 2),(1 \rightarrow 3),(1 \rightarrow 4)$ and $(1 \rightarrow 6)$ sulfonamide-bridged glycomimetics. The chemistry is compatible with larger sugars, variable stereochemical arrangements and acid- and base-labile protecting groups. This chemistry, in combination with the impressive pharmaceutical pedigree of the sulfonamide moiety, featuring in the chemical structure of $>100$ marketed drugs, ${ }^{32}$ affirms the versatility of the sulfonamide moiety as a synthetically universal linker between carbohydrate building blocks. This implies tremendous future potential for the application of 
sulfonamide-bridged glycomimetics in drug discovery and glycobiology, wherein it is desirable to introduce an unnatural link in place of the native glycosidic bond.

\section{Experimental Section}

All starting materials were purchased from commercial suppliers. $S$-acetyl thioglycoses were synthesized according to literature methods. ${ }^{27}$ Amino sugar reagents were either purchased from commercial suppliers or synthesized according to literature methods. ${ }^{33-39}$ All reactions were monitored by TLC using 4 silica plates with visualization of product bands by UV fluorescence $(\lambda=$ $254 \mathrm{~nm}$ ) and charring with orcinol stain (1 $\mathrm{g}$ of orcinol monohydrate in a mixture of EtOH: $\mathrm{H}_{2} \mathrm{O}: \mathrm{H}_{2} \mathrm{SO}_{4}$ 72.5:22.5:5). Silica gel flash chromatography was performed using silica gel 60 $\AA$ (230-400 mesh). NMR $\left({ }^{1} \mathrm{H},{ }^{13} \mathrm{C}\left\{{ }^{1} \mathrm{H}\right\}\right.$, gCOSY and HSQC) spectra were recorded on a $500 \mathrm{MHz}$ spectrometer at $30{ }^{\circ} \mathrm{C}$. Chemical shifts for ${ }^{1} \mathrm{H}$ and ${ }^{13} \mathrm{C}$ NMR acquired in DMSO- $d_{6}$ are reported in ppm relative to residual solvent proton $(\delta=2.50 \mathrm{ppm})$ and carbon $(\delta=39.5 \mathrm{ppm})$ signals, respectively. Chemical shifts for ${ }^{1} \mathrm{H}$ NMR acquired in $\mathrm{CDCl}_{3}$ are reported in ppm relative to residual solvent proton $(\delta=7.26 \mathrm{ppm})$. Multiplicity is indicated as follows: s (singlet); $\mathrm{d}$ (doublet); $\mathrm{t}$ (triplet); m (multiplet); dd (doublet of doublet); ddd (doublet of doublet of doublet); br (broad). Coupling constants are reported in Hertz $(\mathrm{Hz})$. Numbering of di-, tri- and tetrasaccharides used for NMR assignments is shown below.
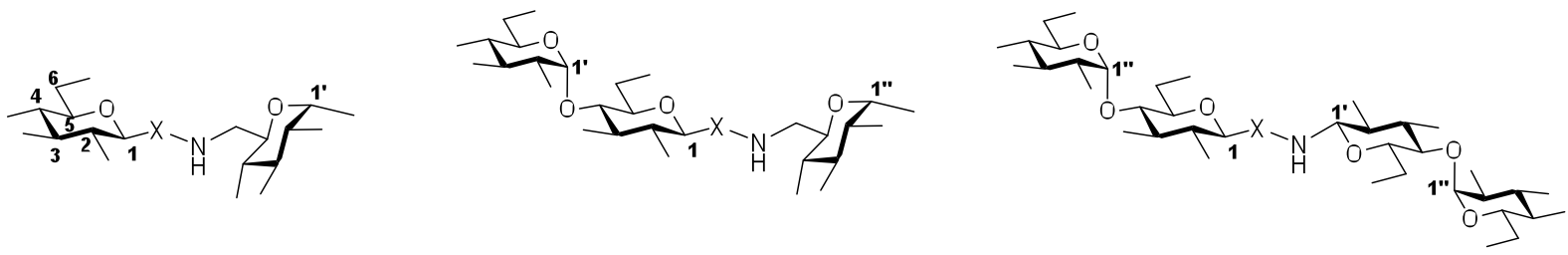

Melting points are uncorrected. High- and low- resolution mass spectra were acquired using electrospray as the ionization technique in positive ion and/or negative ion modes as stated. All MS 
analysis samples were prepared as solutions in methanol. Optical rotations were measured at $25^{\circ} \mathrm{C}$ with Na-589 $\mathrm{nm}$ wave length and a $100 \mathrm{~mm}$ cell and reported as an average of ten measurements.

General procedure 1 - Synthesis of glycomimetic sulfenamides. The thioacetate derivative (1.0 equiv.) was solubilized in anhydrous methanol under a nitrogen atmosphere. Diethylbromomalonate (2.5 equiv.) was added and the reaction stirred for $30 \mathrm{~min}$ at $\mathrm{rt}$ under nitrogen. Next a solution of amino sugar (5.0 equiv.) in methanol, (neutralized with diisopropyl ethylamine (5.0 equiv.) if amino sugar is its hydrochloride salt) was added and the reaction stirred overnight at $40{ }^{\circ} \mathrm{C}$ under nitrogen. For products 10a, 19a, 20a and 22a the methanol was evaporated and the residue solubilized in $\mathrm{CH}_{2} \mathrm{Cl}_{2}$ and washed with brine. After back extraction of each aqueous phase with $\mathrm{CH}_{2} \mathrm{Cl}_{2}$ the organic fractions were combined, dried over $\mathrm{MgSO}_{4}$, filtered and concentrated. For the remaining sulfenamide compounds no aqueous wash was performed due to their high water solubility. Instead these products were purified directly following removal of methanol in the presence of silica gel. The sulfenamides were purified as described for each compound 10a-20a and 22a.

General procedure 2 - Synthesis of glycomimetic sulfonamides. The sulfenamide derivative (1.0 equiv.) was solubilized in $\mathrm{CH}_{2} \mathrm{Cl}_{2}$. $m$-CPBA (7.0 equiv.) as a solution in $\mathrm{CH}_{2} \mathrm{Cl}_{2}$ was added dropwise. The reaction was maintained at $\mathrm{rt}$ with stirring until full disappearance of the starting material, typically $1 \mathrm{~h}$. For products $\mathbf{1 0 b}, \mathbf{1 9 b}, \mathbf{2 0 b}$ and $\mathbf{2 2 b}$ the reaction mixture was diluted in $\mathrm{CH}_{2} \mathrm{Cl}_{2}$ and quenched with saturated $\mathrm{NaHCO}_{3}$. The resulting mixture was filtered through celite. After extraction with $\mathrm{CH}_{2} \mathrm{Cl}_{2}$, the organic layer was washed with saturated $\mathrm{NaHCO}_{3}$ and brine. The aqueous phases were back extracted with $\mathrm{CH}_{2} \mathrm{Cl}_{2}$. The organic fractions were combined, dried over $\mathrm{MgSO}_{4}$, filtered and concentrated. For the remaining sulfonamide compounds no aqueous wash was performed due to their high water solubility. Instead the reaction mixture was concentrated at $10^{\circ} \mathrm{C}$ 
prior to purification. The expected sulfonamides were purified as described for each compound 10b-20b and 22b.

General procedure 3 - Deprotection of per- $\boldsymbol{O}$-acetylated glycomimetics. ${ }^{30}$ Fully deprotected compounds were prepared by treating a solution of the per- $O$-acetylated compound (1.0 equiv.) in $\mathrm{MeOH}$ at $0{ }^{\circ} \mathrm{C}$ with methanolic sodium methoxide (0.05 $\mathrm{M}$ final concentration); $\mathrm{pH} 12$. The reaction was allowed to warm to rt and left to stir until full deprotection was evidenced by TLC (30 min - overnight). The reaction mixture was neutralized with Amberlite IR-120 $\left[\mathrm{H}^{+}\right]$, filtered and the resin washed several times with methanol. The filtrate was evaporated under reduced pressure and lyophilized to afford the fully deprotected compounds $10 \mathbf{c - 2 0 c}$ and $22 \mathrm{c}$. If needed, compounds were purified by flash chromatography as described.

\section{$N$-(2,3,4,6-Tetra- $O$-acetyl-1-S- $\beta$-D-glucopyranosyl)-2,3,4,6-tetra- $O$-acetyl- $\beta$-D-}

glucopyranosylamine (14a). Compound 14a was obtained from compounds 1 and 6 according to the general procedure 1. After purification by flash chromatography (1:1 EtOAc/hexane) 14a (43\% yield) was obtained as a white solid. $\mathrm{R}_{f}=0.24(1: 1 \mathrm{EtOAc} /$ hexane $) . \mathrm{m} . \mathrm{p} .=125-127{ }^{\circ} \mathrm{C} .[\alpha]^{25}{ }_{\mathrm{D}}=+5$ $\left(c=1.0\right.$, chloroform). ${ }^{1} \mathrm{H}$ NMR $\left(500 \mathrm{MHz}, \mathrm{DMSO}-d_{6}\right): \delta=5.32(\mathrm{t}, J=9.5 \mathrm{~Hz}, 1 \mathrm{H}, \mathrm{H}-3) ; 5.21(\mathrm{t}, J$ $=9.5 \mathrm{~Hz}, 1 \mathrm{H}, \mathrm{H}-3 ”) ; 4.96(\mathrm{t}, J=9.5 \mathrm{~Hz}, 1 \mathrm{H}, \mathrm{H}-2) ; 4.94(\mathrm{~m}, 1 \mathrm{H}, \mathrm{NH}) ; 4.87$ (t, $J=10.0 \mathrm{~Hz}, 1 \mathrm{H}, \mathrm{H}-$ 4); 4.85 (t, $J=10.0 \mathrm{~Hz}, 1 \mathrm{H}, \mathrm{H}-4$ ”); 4.83 (t, $J=9.0 \mathrm{~Hz}, 1 \mathrm{H}, \mathrm{H}-2$ '); 4.66 (d, $J=10.0 \mathrm{~Hz}, 1 \mathrm{H}, \mathrm{H}-1)$; 4.38 (t, $J=9.5 \mathrm{~Hz}, 1 \mathrm{H}, \mathrm{H}-1$ "); 4.17 (dd, $J=12.0,4.0 \mathrm{~Hz}, 1 \mathrm{H}, \mathrm{H}-6 \mathrm{a} ") ; 4.14$ (dd, $J=11.5,4.5 \mathrm{~Hz}$, 1H, H-6a); 4.10 (dd, $J=12.0,2.0$ Hz, 1H, H-6b); 4.05 (dd, $J=12.0,2.0 \mathrm{~Hz}, 1 \mathrm{H}, \mathrm{H}-6 \mathrm{~b}$ ”); 3.96 (m, $1 \mathrm{H}, \mathrm{H}-5) ; 3.89$ (m, 1H, H-5”); 2.06, 2.05, 2.02, 2.01, 1.98, 1.97, 1.93, $1.92\left(8 \times \mathrm{s}, 24 \mathrm{H}, \mathrm{OCOCH}_{3}\right)$, assignments were confirmed by ${ }^{1} \mathrm{H}-{ }^{1} \mathrm{H}$ gCOSY. ${ }^{13} \mathrm{C}$ NMR (125 MHz, DMSO- $\left.d_{6}\right): \delta=170.1,170.0$, 
169.5, 169.2 (2C), 169.0, $168.9\left(\mathrm{OCOCH}_{3}\right) ; 91.3$ (C-1”); 87.4 (C-1); 74.2 (C-5); 73.3 (C-3); 73.0 (C-3”); 71.5 (C-5”); 70.5 (C-2”); 68.1 (C-4”); 67.9 (C-4); 67.3 (C-2); 61.8, 61.7 (C-6, C-6”); 20.5 (3C), 20.4, 20.3 (4C) $\left(\mathrm{OCOCH}_{3}\right)$, assignments were confirmed by ${ }^{1} \mathrm{H}^{-13} \mathrm{C}$ HSQC. LRMS $\left(\mathrm{ESI}^{+}\right)$: $m / z=732[\mathrm{M}+\mathrm{Na}]^{+}$. HRMS: Calcd for $\mathrm{C}_{28} \mathrm{H}_{39} \mathrm{NO}_{18} \mathrm{SNa}$ 732.1780, Found 732.1754.

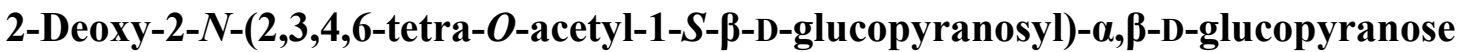

(15a).

Compound 15a was obtained from compound 1 and glucosamine hydrochloride salt 7 according to the general procedure 1. Purification by flash chromatography (2:1 acetone/toluene) afforded 15a (80\% yield) as a mixture of $\alpha$ and $\beta$ anomers $\left(\alpha / \beta\right.$ 80:20) as a light yellow oil. $\mathrm{R}_{f}=0.39$ (2:1 acetone/toluene). ${ }^{1} \mathrm{H}$ NMR (500 MHz, DMSO- $\left.d_{6}\right): \delta=6.48$ (d, $\left.J=6.5 \mathrm{~Hz}, 0.2 \mathrm{H}, \mathrm{OH}-1{ }^{\prime}{ }_{\beta}\right) ; 6.45$ (d, $\left.J=4.5 \mathrm{~Hz}, 0.8 \mathrm{H}, \mathrm{OH}-1{ }_{\alpha}{ }_{\alpha}\right) ; 5.37\left(\mathrm{t}, J=9.5 \mathrm{~Hz}, 0.8 \mathrm{H}, \mathrm{H}-3_{\alpha}\right) ; 5.28\left(\mathrm{t}, J=9.0 \mathrm{~Hz}, 0.2 \mathrm{H}, \mathrm{H}-3_{\beta}\right) ; 5.21$ (br t, $\left.J=4.0 \mathrm{~Hz}, 0.8 \mathrm{H}, \mathrm{H}-1{ }^{\prime}{ }_{\alpha}\right) ; 5.06$ (t, $\left.J=9.5 \mathrm{~Hz}, 0.8 \mathrm{H}, \mathrm{H}-2_{\alpha}\right) ; 4.98$ (t, $\left.J=9.5 \mathrm{~Hz}, 0.8 \mathrm{H}, \mathrm{H}-4_{\alpha}\right)$; $4.93\left(\mathrm{t}, J=10.0 \mathrm{~Hz}, 0.2 \mathrm{H}, \mathrm{H}-2_{\beta}\right) ; 4.90\left(\mathrm{t}, J=9.0 \mathrm{~Hz}, 0.2 \mathrm{H}, \mathrm{H}-4_{\beta}\right) ; 4.74(\mathrm{~d}, J=5.5 \mathrm{~Hz}, 0.8 \mathrm{H}, \mathrm{OH}-$ $\left.4{ }_{\alpha}\right) ; 4.69\left(\mathrm{~d}, J=10.5 \mathrm{~Hz}, 0.2 \mathrm{H}, \mathrm{H}-1_{\beta}\right) ; 4.64\left(\mathrm{~d}, J=5.0 \mathrm{~Hz}, 0.8 \mathrm{H}, \mathrm{OH}-3{ }_{\alpha}\right) ; 4.46(\mathrm{~d}, J=4.5 \mathrm{~Hz}$, $\left.0.2 \mathrm{H}, \mathrm{O} H-3{ }_{\beta}\right) ; 4.44\left(\mathrm{~d}, J=6.5 \mathrm{~Hz}, 0.2 \mathrm{H}, \mathrm{OH}-4{ }_{\beta}\right) ; 4.37$ (d, $\left.J=10.0 \mathrm{~Hz}, 0.8 \mathrm{H}, \mathrm{H}-1_{\alpha}\right) ; 4.36(\mathrm{~m}$, $\left.0.2 \mathrm{H}, \mathrm{H}-1{ }^{\prime} \beta\right) ; 4.27\left(\mathrm{t}, J=5.5 \mathrm{~Hz}, 0.8 \mathrm{H}, \mathrm{OH}-6{ }_{\alpha}{ }_{\alpha}\right) ; 4.13\left(\mathrm{dd}, J=12.5,5.0 \mathrm{~Hz}, 0.2 \mathrm{H}, \mathrm{H}-6 \mathrm{a}_{\beta}\right) ; 4.05(\mathrm{~m}$, 1.6H, H-6 $\left.6_{\alpha}\right) ; 3.98\left(\mathrm{td}, J=10.0,3.0 \mathrm{~Hz}, 0.8 \mathrm{H}, \mathrm{H}-5_{\alpha}\right) ; 3.93\left(\mathrm{~m}, 0.2 \mathrm{H}, \mathrm{H}-5_{\beta}\right) ; 3.89$ (d, $J=5.0 \mathrm{~Hz}$, $\left.0.2 \mathrm{H}, \mathrm{N} H_{\beta}\right) ; 3.66\left(\mathrm{dd}, J=9.5,5.0 \mathrm{~Hz}, 0.2 \mathrm{H}, \mathrm{H}-6 \mathrm{aa}{ }_{\beta}\right) ; 3.58\left(\mathrm{dd}, J=11.0,2.0 \mathrm{~Hz}, 0.8 \mathrm{H}, \mathrm{H}-6 \mathrm{a}{ }_{\alpha}\right) ; 3.04$ (m, 1H, H-4"); 2.95 (d, $\left.J=8.5 \mathrm{~Hz}, 0.8 \mathrm{H}, \mathrm{N} H_{\alpha}\right)$ (m, 1H, H-5"); 3.45 (m, 1H, H-6"b); 3.41 (m, 0.2H, $\left.\mathrm{H}-3{ }^{\prime}{ }^{3}\right) ; 3.25$ (m, 0.8H, H-3” $\left.{ }_{\alpha}\right) ; 2.45$ (m, 0.8H, H-2” $\left.{ }_{\alpha}\right) ; 2.33$ (td, $\left.J=10.0,5.0 \mathrm{~Hz}, 0.2 \mathrm{H}, \mathrm{H}-2{ }^{\prime}{ }_{\beta}\right) ; 2.05$, 2.04, 2.03, 1.99, 1.98, 1.97, $1.96\left(8 \times \mathrm{s}, 12 \mathrm{H}, \mathrm{OCOCH}_{3}\right)$, assignments were confirmed by ${ }^{1} \mathrm{H}-{ }^{1} \mathrm{H}$ gCOSY. ${ }^{13} \mathrm{C}$ NMR (125 MHz, DMSO- $\left.d_{6}\right): \delta=170.3,169.6,169.5,169.2\left(\mathrm{OCOCH}_{3}\right) ; 95.6\left(\mathrm{C}-1{ }^{\prime \prime}\right)$; $91.6\left(\mathrm{C}-1{ }_{\alpha}\right) ; 88.7\left(\mathrm{C}-1_{\beta}\right) ; 86.8\left(\mathrm{C}-1_{\alpha}\right) ; 76.6\left(\mathrm{C}-5_{\beta}\right) ; 74.3\left(\mathrm{C}-5_{\alpha}\right) ; 73.3\left(\mathrm{C}-3_{\beta}\right) ; 73.0\left(\mathrm{C}-3_{\alpha}, \mathrm{C}-3{ }_{\alpha}{ }_{\alpha}, \mathrm{C}-\right.$ 
3” $\left.{ }_{\beta}\right) ; 71.8\left(\mathrm{C}-5_{\alpha}, \mathrm{C}-5{ }_{\beta}\right) ; 70.9\left(\mathrm{C}-4{ }_{\alpha}\right) ; 70.7\left(\mathrm{C}-4{ }_{\beta}\right) ; 69.1\left(\mathrm{C}-2{ }_{\alpha}, \mathrm{C}-2{ }_{\beta}\right) ; 68.1\left(\mathrm{C}-4_{\alpha}\right) ; 67.6\left(\mathrm{C}-4_{\beta}\right)$; $67.3\left(\mathrm{C}-2_{\alpha}\right) ; 66.9\left(\mathrm{C}-2_{\beta}\right) ; 61.8\left(\mathrm{C}-6_{\alpha}, \mathrm{C}-6_{\beta}\right) ; 61.3\left(\mathrm{C}-6{ }_{\alpha}, \mathrm{C}-6{ }^{\prime}{ }\right) ; 20.5(2 \mathrm{C}), 20.4,20.3\left(\mathrm{OCOCH}_{3}\right)$, assignments were confirmed by ${ }^{1} \mathrm{H}^{13}{ }^{13} \mathrm{C}$ HSQC. LRMS $\left(\mathrm{ESI}^{+}\right): m / z=564[\mathrm{M}+\mathrm{Na}]^{+}$. HRMS: Calcd for $\mathrm{C}_{20} \mathrm{H}_{31} \mathrm{NO}_{14} \mathrm{SNa}$ 564.1357, Found 564.1353.

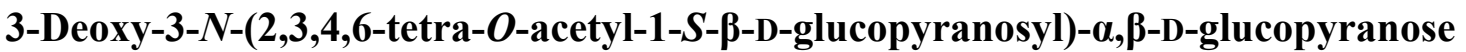

(16a).

Compound 16a was obtained from compound $\mathbf{1}$ and amine $\mathbf{8}$ according to the general procedure 1 . Purification by flash chromatography (5:2 acetone/petroleum spirit) afforded $\mathbf{1 6 a}$ ( $27 \%$ yield) as a mixture of $\alpha$ and $\beta$ anomers $(\alpha / \beta 35: 65)$ as a light yellow gum. $\mathrm{R}_{f}=0.16$ (5:2 acetone/petroleum spirit). ${ }^{1} \mathrm{H}$ NMR (500 MHz, DMSO- $\left.d_{6}\right): \delta=6.63\left(\mathrm{~d}, J=6.0 \mathrm{~Hz}, 0.6 \mathrm{H}, \mathrm{OH}-1{ }^{\prime}{ }^{\prime}\right) ; 6.27(\mathrm{~d}, J=4.5 \mathrm{~Hz}$, $\left.0.4 \mathrm{H}, \mathrm{OH}-\mathrm{1}_{\alpha}{ }_{\alpha}\right) ; 5.30\left(\mathrm{t}, J=9.5 \mathrm{~Hz}, 0.6 \mathrm{H}, \mathrm{H}-3_{\beta}\right) ; 5.29\left(\mathrm{t}, J=9.5 \mathrm{~Hz}, 0.4 \mathrm{H}, \mathrm{H}-3_{\alpha}\right) ; 5.03(\mathrm{t}, J=9.5 \mathrm{~Hz}$, 0.4H, H-2 $\alpha$ ); 4.94 (m, 0.4H, H-1 $\left.)_{\alpha}\right) ; .92\left(\mathrm{~m}, 1 \mathrm{H}, \mathrm{H}-4_{\alpha}, \mathrm{H}-4_{\beta}\right) ; 4.91$ (t, $\left.J=10.0 \mathrm{~Hz}, 0.6 \mathrm{H}, \mathrm{H}-2_{\beta}\right) ; 4.71$ (d, $\left.J=10.0 \mathrm{~Hz}, 0.6 \mathrm{H}, \mathrm{H}-1_{\beta}\right) ; 4.65\left(\mathrm{~d}, J=10.0 \mathrm{~Hz}, 0.4 \mathrm{H}, \mathrm{H}-1_{\alpha}\right) ; 4.64\left(\mathrm{~d}, J=5.0 \mathrm{~Hz}, 0.6 \mathrm{H}, \mathrm{OH}-2{ }^{\prime}{ }_{\beta}\right)$; 4.48-4.46 (m, 1.2H, OH-6 $\left.{ }_{\beta}, \mathrm{O} H-4{ }^{\prime}\right) ; 4.38\left(\mathrm{~d}, J=5.5 \mathrm{~Hz}, 0.4 \mathrm{H}, \mathrm{OH}-4{ }^{\prime}{ }_{\alpha}\right) ; 4.35(\mathrm{t}, J=6.0 \mathrm{~Hz}, 0.4 \mathrm{H}$, $\left.\mathrm{O} H-6^{\prime}{ }_{\alpha}\right) ; 4.31\left(\mathrm{dd}, J=7.5,6.5 \mathrm{~Hz}, 0.6 \mathrm{H}, \mathrm{H}-{ }^{\prime}{ }_{\beta}\right) ; 4.15-4.11\left(\mathrm{~m}, 2 \mathrm{H}, \mathrm{OH}-2^{\prime}{ }_{\alpha}, \mathrm{H}-6 \mathrm{a}_{\alpha}, \mathrm{H}-6 \mathrm{a}_{\beta}, \mathrm{N}_{\beta}\right)$; 4.05-4.01 (m, 1.9H, H-6b $\left.\mathrm{b}_{\alpha}, \mathrm{H}-6 \mathrm{~b}_{\beta}\right)$; 3.98-3.92 (m, $\left.1 \mathrm{H}, \mathrm{H}-5_{\alpha}, \mathrm{H}-5_{\beta}\right) ; 3.81$ (d, $\left.J=5.0 \mathrm{~Hz}, 0.4 \mathrm{H}, \mathrm{N} H_{\alpha}\right)$;

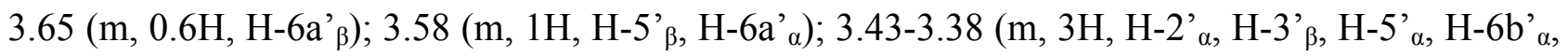
H-6b' $\left.{ }_{\beta}\right) ; 3.21-3.16$ (m, $\left.1 \mathrm{H}, \mathrm{H}-4{ }_{\alpha}{ }_{\alpha}, \mathrm{H}-4{ }_{\beta}{ }_{\beta}\right) ; 3.10$ (m, 0.6H, H-2 $\left.{ }_{\beta}\right) ; 2.81$ (m, 0.4, H-3 $\left.{ }_{\alpha}{ }_{\alpha}\right) ; 2.03,2.02$, 2.01, 1.98, $1.95\left(6 \times \mathrm{s}, 12 \mathrm{H}, \mathrm{OCOCH}_{3}\right)$, assignments were confirmed by ${ }^{1} \mathrm{H}-{ }^{1} \mathrm{H}$ gCOSY.${ }^{13} \mathrm{C}$ NMR (125 MHz, DMSO- $d_{6}, \beta$-anomer): $\delta=170.6,170.1,169.8,169.7\left(\mathrm{OCOCH}_{3}\right) ; 98.0(\mathrm{C}-1$ ') $; 89.3(\mathrm{C}-$ 1); 78.1 (C-5'); 74.8 (C-5); 73.8 (C-3); 71.8 (C-2'); 68.6, 68.3, 67.9 (C-2, C-4, C-4'); 64.8 (C-3'); $62.4(\mathrm{C}-6) ; 61.8\left(\mathrm{C}-6^{\prime}\right) ; 21.1,21.0,20.8(2 \mathrm{C})\left(\mathrm{OCOCH}_{3}\right)$, assignments were confirmed by ${ }^{1} \mathrm{H}^{13}{ }^{13} \mathrm{C}$ 
HSQC. LRMS $\left(\mathrm{ESI}^{+}\right): m / z=564[\mathrm{M}+\mathrm{Na}]^{+}$. HRMS: Calcd for $\mathrm{C}_{20} \mathrm{H}_{31} \mathrm{NO}_{14} \mathrm{SNa}$ 564.1367, Found 564.1383.

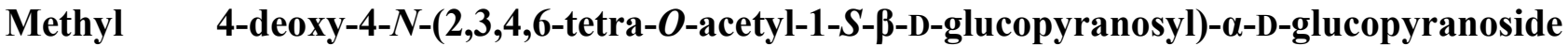

(17a). Compound 17a was prepared from compounds 1 and 9 according to the general procedure 1. Purification by flash chromatography (2:1 acetone/hexane) afforded the title compound as a light yellow gum. (73\% yield). $\mathrm{R}_{f}=0.21\left(2: 1\right.$ acetone/hexane). $[\alpha]^{25}=+22\left(c=1.0\right.$, chloroform). ${ }^{1} \mathrm{H}$ NMR (500 MHz, DMSO- $\left.d_{6}\right): \delta=5.30$ (t, $\left.J=9.5 \mathrm{~Hz}, 1 \mathrm{H}, \mathrm{H}-3\right) ; 4.99$ (t, $\left.J=9.5 \mathrm{~Hz}, 1 \mathrm{H}, \mathrm{H}-2\right) ; 4.88$ (t, $J=9.5 \mathrm{~Hz}, 1 \mathrm{H}, \mathrm{H}-4) ; 4.69$ (d, $J=6.5 \mathrm{~Hz}, 1 \mathrm{H}, \mathrm{OH}-2$ "); 4.60 (d, $J=10.0 \mathrm{~Hz}, 1 \mathrm{H}, \mathrm{H}-1) ; 4.56$ (d, $J$ $=6.0 \mathrm{~Hz}, 1 \mathrm{H}, \mathrm{OH}-3$ ”); $4.51(\mathrm{~d}, J=3.5 \mathrm{~Hz}, 1 \mathrm{H}, \mathrm{H}-1$ "); 4.35 (t, $J=6.0 \mathrm{~Hz}, 1 \mathrm{H}, \mathrm{OH}-6$ "); $4.16(\mathrm{dd}, J=$ $12.0,5.0 \mathrm{~Hz}, 1 \mathrm{H}, \mathrm{H}-6 \mathrm{a}) ; 4.02$ (dd, $J=12.0,2.0 \mathrm{~Hz}, 1 \mathrm{H}, \mathrm{H}-6 \mathrm{~b}) ; 3.96$ (ddd, $J=10.0,5.0,2.0 \mathrm{~Hz}, 1 \mathrm{H}$, H-5); 3.84 (d, J=5.0 Hz, 1H, NH); 3.67 (m, 1H, H-6a”); 3.55 (m, 1H, H-3”); 3.51 (dd, J= 11.5, 5.5 Hz, 1H, H-6b"); 3.48 (m, 1H, H-5”); 3.24 (s, 3H, OCH ( $_{3} ; 3.20$ (ddd, J=10.0, 6.5, 3.5 Hz, 1H, H2”); $2.54(\mathrm{td}, J=10.0,5.5 \mathrm{~Hz}, 1 \mathrm{H}, \mathrm{H}-4 ”) ; 2.02,2.01,1.98,1.95\left(4 \times \mathrm{s}, 12 \mathrm{H}, \mathrm{OCOCH}_{3}\right)$, assignments were confirmed by ${ }^{1} \mathrm{H}-{ }^{1} \mathrm{H}$ gCOSY.${ }^{13} \mathrm{C}$ NMR $\left(125 \mathrm{MHz}, \mathrm{DMSO}-d_{6}\right): \delta=170.0,169.5$, 169.3, 169.2 ( $\left.\mathrm{OCOCH}_{3}\right)$; 99.6 (C-1”); 87.3 (C-1); 74.3 (C-5); 73.2 (C-3); 72.8 (C-2”); 71.4 (C-5”); 70.6 (C-3”); 68.1 (C-4); 67.6 (C-2); 62.1 (C-6); 62.0 (C-4”); 61.1 (C-6”); $54.2\left(\mathrm{OCH}_{3}\right) ; 20.7,20.5$, 20.3 (2C) $\left(\mathrm{OCOCH}_{3}\right)$, assignments were confirmed by ${ }^{1} \mathrm{H}^{13}{ }^{13} \mathrm{HSQC}$. LRMS $\left(\mathrm{ESI}^{+}\right): \mathrm{m} / z=555$ [M $+\mathrm{H}]^{+}, 578[\mathrm{M}+\mathrm{Na}]^{+} . \mathrm{HRMS}$ : Calcd for $\mathrm{C}_{21} \mathrm{H}_{33} \mathrm{NO}_{14} \mathrm{SNa}$ 578.1514, Found 578.1491.

\section{Methyl 6-deoxy-6- $N-(2,3,4,6$-tetra- $O$-acetyl-1-S- $\beta$-D-glucopyranosyl)- $\alpha$-D-glucopyranoside}

(18a). Compound 18a was obtained from compounds 1 and 10 according to the general procedure 1. Purification by flash chromatography (2:1 acetone/hexane) afforded 18a as a light yellow gum 
(90\% yield). $\mathrm{R}_{f}=0.30\left(2: 1\right.$ acetone/hexane). LRMS $\left(\mathrm{ESI}^{+}\right): m / z=556[\mathrm{M}+\mathrm{H}]^{+}$. HRMS: Calcd for $\mathrm{C}_{21} \mathrm{H}_{33} \mathrm{NO}_{14} \mathrm{SNa}$ 578.1514, Found 578.1504. Compound 18a was used as reagent for general procedure 3 without further purification.

\section{$N$-(2,3,4,6-Tetra- $O$-acetyl-1-S- $\beta$-D-glucopyranosyl)-2,3,4,6-tetra- $O$-acetyl- $\beta$-D-}

glucopyranosysulfonylamine (14b). Compound 14b was obtained from compound 14a according to the general procedure 2. Purification by flash chromatography (1:2 EtOAc/hexane) afforded $\mathbf{1 4 b}$ (65\% yield) as a white solid. $\mathrm{R}_{f}=0.39(1: 1 \mathrm{EtOAc} /$ hexane $) . \mathrm{m} . \mathrm{p} .=90-93{ }^{\circ} \mathrm{C} \cdot[\alpha]^{25}{ }_{\mathrm{D}}=+4(c=1.0$, chloroform). ${ }^{1} \mathrm{H}$ NMR (500 MHz, DMSO- $\left.d_{6}\right): \delta=9.14($ br s, $1 \mathrm{H}, \mathrm{NH}) ; 5.42(\mathrm{t}, J=9.0 \mathrm{~Hz}, 1 \mathrm{H}, \mathrm{H}-$ 3); 5.39 (t, $J=9.0 \mathrm{~Hz}, 1 \mathrm{H}, \mathrm{H}-3$ '); 5.25 (t, $J=9.0 \mathrm{~Hz}, 1 \mathrm{H}, \mathrm{H}-2$ '); 4.93-4.87 (m, 4H, H-1, H-2', H-4, H-4'); 4.74 (br t, $J=9.0 \mathrm{~Hz}, 1 \mathrm{H}, \mathrm{H}-1^{\prime}$ ); 4.23 (dd, $\left.J=12.5,3.5 \mathrm{~Hz}, 1 \mathrm{H}, \mathrm{H}-6 \mathrm{a}\right) ; 4.18-4.10$ (m, 3H, H6b, H-6a', H-6b'); 4.07 (m, 1H, H-5'); 4.03 (m, 1H, H-5); 2.04, 2.01, 2.00, 1.99, 1.98, 1.97, 1.93 (7 $\left.\times \mathrm{s}, 24 \mathrm{H}, \mathrm{OCOCH}_{3}\right)$, assignments were confirmed by ${ }^{1} \mathrm{H}-{ }^{1} \mathrm{H}$ gCOSY. ${ }^{13} \mathrm{C}$ NMR (125 MHz, DMSO$\left.d_{6}\right): \delta=170.5,170.0,169.9,169.8,169.6,169.5,169.2,169.1\left(\mathrm{OCOCH}_{3}\right) ; 87.1\left(\mathrm{C}-1{ }^{\prime}\right) ; 81.8(\mathrm{C}-1)$; 74.7 (C-5); 73.5 (C-2); $73.2,72.5$ (C-3, C-3’); 70.8 (C-2'); 72.5 (C-5’); 67.8, 67.5 (C-4, C-4'); 62.2 (C-6'); 61.7 (C-6); 21.1, 21.0, 20.9 (2C), 20.8 (2C). 20.7, $20.6\left(\mathrm{OCOCH}_{3}\right)$, assignments were confirmed by ${ }^{1} \mathrm{H}_{-}{ }^{13} \mathrm{C}$ HSQC. LRMS $\left(\mathrm{ESI}^{+}\right): m / z=764[\mathrm{M}+\mathrm{Na}]^{+}$. HRMS: Calcd for $\mathrm{C}_{28} \mathrm{H}_{39} \mathrm{NO}_{20} \mathrm{SNa} 764.1678$, Found 764.1653.

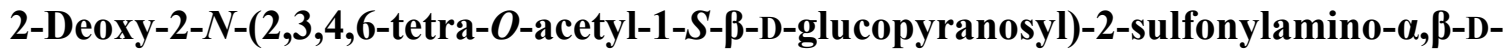

glucopyranose (15b). Compound $\mathbf{1 5 b}$ was obtained from compound $\mathbf{1 5 a}$ according to the general procedure 2. Purification by flash chromatography ( $1: 1$ acetone/toluene) afforded the title compound (71\% yield) as a mixture of $\alpha$ and $\beta$ anomers $\left(\alpha / \beta\right.$ 85:15) as a white solid. $\mathrm{R}_{f}=0.39(2: 1$ 
acetone/toluene). m.p. $=169-171{ }^{\circ} \mathrm{C} .{ }^{1} \mathrm{H}$ NMR $\left(500 \mathrm{MHz}\right.$, DMSO- $d_{6}, \beta$-anomer $): \delta=6.71(\mathrm{~d}, J=$ $8.5 \mathrm{~Hz}, 1 \mathrm{H}, \mathrm{NH}) ; 6.66\left(\mathrm{~d}, J=4.0 \mathrm{~Hz}, 1 \mathrm{H}, \mathrm{OH}-1^{\prime}\right) ; 5.38(\mathrm{t}, J=9.5 \mathrm{~Hz}, 1 \mathrm{H}, \mathrm{H}-3) ; 5.29(\mathrm{t}, J=9.5 \mathrm{~Hz}$ 1H, H-2); 5.06 (t, $\left.J=4.0 \mathrm{~Hz}, 1 \mathrm{H}, \mathrm{H}-1^{\prime}\right) ; 5.02$ (t, $\left.J=9.5 \mathrm{~Hz}, 1 \mathrm{H}, \mathrm{H}-4\right) ; 4.95$ (d, $J=5.5 \mathrm{~Hz}, 1 \mathrm{H}, \mathrm{OH}-$ 3' or $\left.\mathrm{OH}-4^{\prime}\right) ; 4.92(\mathrm{~d}, J=9.5 \mathrm{~Hz}, 1 \mathrm{H}, \mathrm{H}-1) ; 4.63\left(\mathrm{~d}, J=5.0 \mathrm{~Hz}, 1 \mathrm{H}, \mathrm{OH}-4^{\prime}\right.$ or $\left.\mathrm{OH}-3^{\prime}\right) ; 4.34(\mathrm{t}, J=$ $\left.6.0 \mathrm{~Hz}, 1 \mathrm{H}, \mathrm{OH}-6^{\prime}\right) ; 4.18$ (dd, $\left.J=12.0,4.0 \mathrm{~Hz}, 1 \mathrm{H}, \mathrm{H}-6 \mathrm{a}\right) ; 4.12$ (ddd, $J=10.0,5.5,3.5,10.0 \mathrm{~Hz}$, 1H, H-5); 4.07 (dd, $J=12.0,2.0 \mathrm{~Hz}, 1 \mathrm{H}, \mathrm{H}-6 \mathrm{~b}) ; 3.61$ (dd, $J=11.5,5.0 \mathrm{~Hz}, 1 \mathrm{H}, \mathrm{H}-6 \mathrm{a}$ '); 3.57 (m, 1H, H-5'). 3.48 (m, H-4' or H-3'); 3.46 (dd, $J=11.0,5.5$ Hz, 1H, H-6b”); 3.12 (m, 1H, H-3' or H4'); $2.04,1.98,1.97,1.96\left(8 \times \mathrm{s}, 12 \mathrm{H}, \mathrm{OCOCH}_{3}\right)$, assignments were confirmed by ${ }^{1} \mathrm{H}-{ }^{1} \mathrm{H}$ gCOSY. ${ }^{13} \mathrm{C}$ NMR $\left(125 \mathrm{MHz}, \mathrm{DMSO}-d_{6}\right): \delta=170.2,169.5,169.3,169.1\left(\mathrm{OCOCH}_{3}\right) ; 91.7(\mathrm{C}-1$ ') $; 86.4(\mathrm{C}-$ 1); 74.5 (C-5); 72.6 (C-3); 71.9 (C-5'); 70.8 (C-3' or C-4'); 70.6 (C-4' or C-3'); 67.6 (C-2); 67.4 (C-4); 61.3 (C-6); 61.1 (C-6'); 58.7 (C-2'); 20.5, 20.4, 20.3, $20.2\left(\mathrm{OCOCH}_{3}\right)$, assignments were confirmed by ${ }^{1} \mathrm{H}^{13}{ }^{13} \mathrm{C}$ HSQC. LRMS $\left(\mathrm{ESI}^{+}\right): m / z=596[\mathrm{M}+\mathrm{Na}]^{+}$; LRMS (ESI $): m / z=572[\mathrm{M}-\mathrm{H}]^{-}$ . HRMS: Calcd for $\mathrm{C}_{20} \mathrm{H}_{31} \mathrm{NO}_{16} \mathrm{SNa} 596.1256$, Found 596.1245.

\section{3-Deoxy-3- $N$-(2,3,4,6-tetra- $O$-acetyl-1-S- $\beta$-D-glucopyranosyl)-3-sulfonylamino- $\alpha, \beta$-D-}

glucopyranose (16b). Compound $\mathbf{1 6 b}$ was obtained from compound $16 \mathrm{a}$ according to the general procedure 2. Purification by flash chromatography $\left(92: 8 \mathrm{CH}_{2} \mathrm{Cl}_{2} / \mathrm{MeOH}\right)$ afforded $\mathbf{1 6 b}$ (79\% yield) as a mixture of $\alpha$ and $\beta$ anomers $(\alpha / \beta 50: 50)$ as a colorless gum. $\mathrm{R}_{f}=0.19\left(92: 8 \mathrm{CH}_{2} \mathrm{Cl}_{2} / \mathrm{MeOH}\right) .{ }^{1} \mathrm{H}$ NMR (500 MHz, DMSO- $\left.d_{6}\right): \delta=7.65\left(\mathrm{~d}, J=7.5 \mathrm{~Hz}, 0.5 \mathrm{H}, \mathrm{N} H_{\beta}\right) ; 7.53\left(\mathrm{~d}, J=7.0 \mathrm{~Hz}, 0.5 \mathrm{H}, \mathrm{N} H_{\alpha}\right)$; $6.68\left(\mathrm{~d}, J=6.5 \mathrm{~Hz}, 0.5 \mathrm{H}, \mathrm{O} H-1_{\beta}\right) ; 6.41\left(\mathrm{~d}, J=4.5 \mathrm{~Hz}, 0.5 \mathrm{H}, \mathrm{O} H-1_{\alpha}\right) ; 5.25\left(\mathrm{t}, J=9.5 \mathrm{~Hz}, 1 \mathrm{H}, \mathrm{H}-3_{\alpha}\right.$, $\left.\mathrm{H}-3_{\beta}\right) ; 5.24,5.20\left(2 \times \mathrm{t}, J=9.5 \mathrm{~Hz}, 1 \mathrm{H}, \mathrm{H}-2_{\alpha}, \mathrm{H}-2_{\beta}\right) ; 4.96,4.95\left(2 \times \mathrm{t}, J=9.0 \mathrm{~Hz}, 1 \mathrm{H}, \mathrm{H}-4_{\alpha}, \mathrm{H}-4_{\beta}\right)$; $4.95\left(\mathrm{~d}, J=9.5 \mathrm{~Hz}, 1 \mathrm{H}, \mathrm{H}-1_{\alpha}, \mathrm{H}-1_{\beta}\right) ; 4.93\left(\mathrm{~d}, J=4.5 \mathrm{~Hz}, 0.5 \mathrm{H}, \mathrm{H}-1^{\prime}{ }_{\alpha}\right) ; 4.85(\mathrm{~d}, J=5.5 \mathrm{~Hz}, 1 \mathrm{H}$, $\mathrm{O} H) ; 4.77(\mathrm{~d}, J=7.0 \mathrm{~Hz}, 0.5 \mathrm{H}, \mathrm{OH}) ; 4.56\left(\mathrm{t}, J=6.0 \mathrm{~Hz}, 0.5 \mathrm{H}, \mathrm{OH}-6^{\prime}\right) ; 4.47$ (t, $J=6.0 \mathrm{~Hz}, 0.5 \mathrm{H}$, 
$\left.\mathrm{OH}-6^{\prime}\right) ; 4.30\left(\mathrm{t}, J=6.5 \mathrm{~Hz}, 0.5 \mathrm{H}, \mathrm{H}-1_{\beta}^{\prime}\right) ; 4.23-4.20\left(\mathrm{~m}, 1 \mathrm{H}, \mathrm{H}-6 \mathrm{a}_{\alpha}, \mathrm{H}-6 \mathrm{a}_{\beta}\right) ; 4.18(\mathrm{~d}, J=7.5 \mathrm{~Hz}$, 0.5H, OH) 4.08, $4.05\left(2 \times \mathrm{dd}, J=10.0,2.0 \mathrm{~Hz}, 1 \mathrm{H}, \mathrm{H}-6 \mathrm{~b}_{\alpha}, \mathrm{H}-6 \mathrm{~b}_{\beta}\right) ; 4.06-3.96\left(\mathrm{~m}, 1 \mathrm{H}, \mathrm{H}-5_{\alpha}, \mathrm{H}-5_{\beta}\right)$; 3.68-3.65 (m, 0.5H, H-6a'); 3.60-3.57 (m, 1H, H-5, H-6a'); 3.50 (dd, J=11.5, 5.0 Hz, 0.5H, H$\left.6 b^{\prime}\right) ; 3.46\left(\mathrm{dd}, J=11.5,6.0 \mathrm{~Hz}, 0.5 \mathrm{H}, \mathrm{H}-6 \mathrm{~b}\right.$ '); 3.28 (m, 0.5H, H-3' $\left.{ }_{\alpha}\right)$; 3.23-3.18 (m, 1.5H, H-2 ${ }_{\alpha}, \mathrm{H}-$ $\left.4^{\prime}{ }_{\alpha}, \mathrm{H}-4{ }_{\beta}\right) ; 3.10-3.07$ (m, 0.5H, H-5'); 3.01-2.98 (m, 1H, H-2 $\left.{ }_{\beta}, \mathrm{H}-3{ }^{\prime}{ }_{\beta}\right) ; 2.02,1.98,1.96,1.95,1.94$ $\left(5 \times \mathrm{s}, 12 \mathrm{H}, \mathrm{OCOCH}_{3}\right)$, assignments were confirmed by ${ }^{1} \mathrm{H}-{ }^{1} \mathrm{H}$ gCOSY. ${ }^{13} \mathrm{C} \mathrm{NMR}(125 \mathrm{MHz}$, DMSO- $\left.d_{6}\right): \delta=170.1,169.5,169.1,168.6\left(\mathrm{OCOCH}_{3}\right) ; 97.5\left(\mathrm{C}-1{ }_{\beta}{ }_{\beta}\right) ; 91.7\left(\mathrm{C}-1{ }_{\alpha}{ }_{\alpha}\right) ; 85.6,85.3\left(\mathrm{C}-1_{\alpha}\right.$, C-1 $\left.{ }_{\beta}\right) ; 77.6$ (C-5’); 74.6 (C-5); 73.3 (C-2); 72.4 (C-2’); 69.0,68.9 (C-4 $\left.{ }_{\alpha}{ }_{\alpha}, \mathrm{C}^{\prime}{ }^{\prime}{ }_{\beta}\right) ; 67.7$ (C-3); 67.6 (C-4); 62.5 (C-3’); 61.7, $61.6\left(\mathrm{C}-6_{\alpha}, \mathrm{C}-6_{\beta}\right) ; 61.0,60.9$ (C-6' $\left.{ }_{\alpha}, \mathrm{C}^{\prime} 6_{\beta}^{\prime}\right) ; 20.5$ (2C), 20.3, 20.2 $\left(\mathrm{OCOCH}_{3}\right)$, assignments were confirmed by ${ }^{1} \mathrm{H}_{-}{ }^{13} \mathrm{C}$ HSQC. LRMS $\left(\mathrm{ESI}^{+}\right): m / z=591\left[\mathrm{M}+\mathrm{NH}_{4}\right]^{+}$, $596[\mathrm{M}+\mathrm{Na}]^{+}$. HRMS: Calcd for $\mathrm{C}_{20} \mathrm{H}_{31} \mathrm{NO}_{16} \mathrm{SNa} 596.1256$, Found 596.1255.

\section{Methyl 4-deoxy-4- $N$-(2,3,4,6-tetra- $O$-acetyl-1- $S$ - $\beta$-D-glucopyranosyl)-4-sulfonylamino- $\alpha$-D-} glucopyranoside (17b). Compound $\mathbf{1 7 b}$ was prepared from compound $\mathbf{1 7} \mathbf{a}$ according to the general procedure 2. Purification by flash chromatography $(2: 1$ acetone/hexane) afforded the title compound as a white solid ( $86 \%$ yield). $\mathrm{R}_{f}=0.34\left(2: 1\right.$ acetone/hexane). m.p. $=138-140{ }^{\circ} \mathrm{C} .[\alpha]^{25}=$ $+52(c=1.0$, chloroform $) .{ }^{1} \mathrm{H}$ NMR $\left(500 \mathrm{MHz}, \mathrm{DMSO}-d_{6}\right): \delta=7.83(\mathrm{~d}, J=9.0 \mathrm{~Hz}, 1 \mathrm{H}, \mathrm{NH}) ; 5.23$ (t, $J=9.0 \mathrm{~Hz}, 1 \mathrm{H}, \mathrm{H}-3) ; 5.17$ (t, $J=9.5 \mathrm{~Hz}, 1 \mathrm{H}, \mathrm{H}-2) ; 4.97$ (t, $J=9.5 \mathrm{~Hz}, 1 \mathrm{H}, \mathrm{H}-4) ; 4.96$ (d, $J=$ $10.0 \mathrm{~Hz}, 1 \mathrm{H}, \mathrm{H}-1)$ ); 4.96 (br s, 2H, OH-2', $\mathrm{OH}-3^{\prime}$ ); 4.54 (d, $J=3.5 \mathrm{~Hz}, 1 \mathrm{H}, \mathrm{H}-1^{\prime}$ ); 4.45 (br t, $J=6.0$ Hz, 1H, OH-6'); 4.23 (dd, $J=12.5,4.0 \mathrm{~Hz}, 1 \mathrm{H}, \mathrm{H}-6 \mathrm{a}) ; 4.06$ (dd, $J=11.5,1.5 \mathrm{~Hz}, 1 \mathrm{H}, \mathrm{H}-6 \mathrm{~b}) ; 3.98$ (br dd, $J=10.0,4.0 \mathrm{~Hz}, 1 \mathrm{H}, \mathrm{H}-5) ; 3.67$ (dd, $J=11.0,6.0 \mathrm{~Hz}, 1 \mathrm{H}, \mathrm{H}-6 \mathrm{a}$ '); 3.53 (td, $J=9.5,4.5 \mathrm{~Hz}$, 1H, H-3'); 3.45 (m, 1H, H-5'); 3.37 (dd, $J=12.0,6.0$ Hz, 1H, H-6b'); 3.26 (s, 3H, OCH $)$; 3.21 (m, $\left.1 \mathrm{H}, \mathrm{H}-2^{\prime}\right) ; 2.88\left(\mathrm{~m}, 1 \mathrm{H}, \mathrm{H}-4^{\prime}\right) ; 2.03,1.98,1.95\left(3 \times \mathrm{s}, 12 \mathrm{H}, \mathrm{OCOCH}_{3}\right)$, assignments were 
confirmed by ${ }^{1} \mathrm{H}-{ }^{1} \mathrm{H}$ gCOSY. ${ }^{13} \mathrm{C}$ NMR $\left(125 \mathrm{MHz}\right.$, DMSO- $\left.d_{6}\right): \delta=170.1,169.5,169.1,168.6$ $\left(\mathrm{OCOCH}_{3}\right) ; 99.4\left(\mathrm{C}-1 '^{\prime}\right) ; 85.0$ (C-1); 74.7 (C-5); 73.4 (C-3); 72.9 (C-2'); 71.3 (C-3'); 71.2 (C-5'); 67.6 (C-2); 67.5 (C-4); 61.5 (C-6); 61.0 (C-6’); 56.3 (C-4'); $54.4\left(\mathrm{OCH}_{3}\right) ; 20.5,20.4,20.2,20.2$ $\left(\mathrm{OCOCH}_{3}\right)$, assignments were confirmed by ${ }^{1} \mathrm{H}_{-}{ }^{13} \mathrm{C}$ HSQC. LRMS $\left(\mathrm{ESI}^{+}\right): m / z=605\left[\mathrm{M}+\mathrm{NH}_{4}\right]^{+}$, $610[\mathrm{M}+\mathrm{Na}]^{+}$. HRMS: Calcd for $\mathrm{C}_{21} \mathrm{H}_{33} \mathrm{NO}_{16} \mathrm{SNa}$ 610.1412, Found 610.1385 .

\section{Methyl 6-deoxy-6- $N$-(2,3,4,6-tetra- $O$-acetyl-1-S- $\beta$-D-glucopyranosyl)-6-sulfonylamino- $\alpha$-D-} glucopyranoside (18b). Compound $\mathbf{1 8 b}$ was obtained from compound 18a according to the general procedure 2. Purification by flash chromatography (2:1 acetone/toluene) afforded $\mathbf{1 8 a}$ ( $76 \%$ yield) as a light yellow gum. $\mathrm{R}_{f}=0.44$ (3:1 acetone/toluene). $[\alpha]^{25}=+21\left(c=1.0\right.$, chloroform). ${ }^{1} \mathrm{H}$ NMR (500 MHz, DMSO- $\left.d_{6}\right): \delta=7.43($ br s, $1 \mathrm{H}, \mathrm{N} H) ; 5.33(\mathrm{t}, J=9.5 \mathrm{~Hz}, 1 \mathrm{H}, \mathrm{H}-3) ; 5.19$ (t, $J=9.5 \mathrm{~Hz}$, 1H, H-2); 5.08 (d, $\left.J=5.5 \mathrm{~Hz}, 1 \mathrm{H}, \mathrm{OH}-4^{\prime}\right) ; 4.95\left(\mathrm{~d}, J=4.0 \mathrm{~Hz}, 1 \mathrm{H}, \mathrm{OH}-3^{\prime}\right) ; 4.91(\mathrm{~d}, J=2.0 \mathrm{~Hz}, 1 \mathrm{H}$, $\left.\mathrm{OH}-2^{\prime}\right) ; 4.89$ (t, $\left.J=9.5 \mathrm{~Hz}, 1 \mathrm{H}, \mathrm{H}-4\right) ; 4.82$ (d, $\left.J=10.0 \mathrm{~Hz}, 1 \mathrm{H}, \mathrm{H}-1\right) ; 4.56(\mathrm{~d}, J=3.5 \mathrm{~Hz}, 1 \mathrm{H}, \mathrm{H}-$ 1'); 4.16 (dd, $J=12.0,5.0 \mathrm{~Hz}, 1 \mathrm{H}, \mathrm{H}-6 \mathrm{a}) ; 4.08$ (m, 1H, H-5); 4.04 (dd, $J=12.0,2.0 \mathrm{~Hz}, 1 \mathrm{H}, \mathrm{H}-6 \mathrm{~b}$ ); 3.44 (br d, $\left.J=13.5 \mathrm{~Hz}, 1 \mathrm{H}, \mathrm{H}-6 \mathrm{a}^{\prime}\right) ; 3.36$ (m, 1H, H-3'); 3.34 (m, 1H, H-5'); 3.28 (s, 3H, OCH ); $^{\prime}$ 3.19 (m, 1H, H-2'); 3.03 (dd, $\left.J=13.0,7.5 \mathrm{~Hz}, 1 \mathrm{H}, \mathrm{H}-6 \mathrm{~b}^{\prime}\right) ; 2.95$ (td, $\left.J=9.5,5.5 \mathrm{~Hz}, 1 \mathrm{H}, \mathrm{H}-4^{\prime}\right)$; $1.98,1.96,1.93,1.91\left(4 \times \mathrm{s}, 12 \mathrm{H}, \mathrm{OCOCH}_{3}\right)$, assignments were confirmed by ${ }^{1} \mathrm{H}-{ }^{1} \mathrm{H}$ gCOSY.${ }^{13} \mathrm{C}$ NMR (125 MHz, DMSO- $\left.d_{6}\right): \delta=171.0,170.4,170.1,169.6\left(\mathrm{OCOCH}_{3}\right) ; 100.0\left(\mathrm{C}-1^{\prime}\right) ; 87.0(\mathrm{C}-1)$; 75.1 (C-5); 73.5 (C-3' or C-5'); 73.3 (C-3); 72.3 (C-2'); 72.0 (C-4'); 71.4 (C-3' or C-5'); 68.2 (C2); 68.1 (C-4); $62.2(\mathrm{C}-6) ; 55.1\left(\mathrm{OCH}_{3}\right) ; 45.5\left(\mathrm{C}-6\right.$ ') ; 21.0, 20.9, 20.8, $20.7\left(\mathrm{OCOCH}_{3}\right)$, assignments were confirmed by ${ }^{1} \mathrm{H}^{13}{ }^{13} \mathrm{HSQC}$. LRMS $\left(\mathrm{ESI}^{+}\right): m / z=588[\mathrm{M}+\mathrm{H}]^{+}$. HRMS: Calcd for $\mathrm{C}_{21} \mathrm{H}_{33} \mathrm{NO}_{16} \mathrm{SNa} 610.1412$, Found 610.1413. 


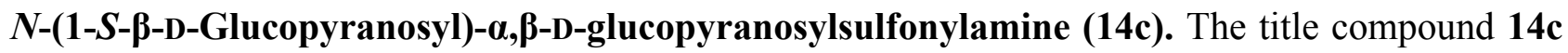
was obtained from compound $\mathbf{1 4 b}$ according to the general procedure 3. Purification by flash chromatography $\left(9: 1 \mathrm{CH}_{3} \mathrm{CN} / \mathrm{H}_{2} \mathrm{O}\right)$ afforded $14 \mathrm{c}$ as a mixture of $\alpha$ and $\beta$ anomers $(72 \%$ yield, $\alpha / \beta$ 70:30) as a clear oil. $\mathrm{R}_{f}=0.15\left(85: 15 \mathrm{CH}_{3} \mathrm{CN} / \mathrm{H}_{2} \mathrm{O}\right) .[\alpha]^{25}{ }_{\mathrm{D}}=+12(c=1.0, \mathrm{MeOH}) .{ }^{1} \mathrm{H}$ NMR $(500$ MHz, DMSO- $\left.d_{6}\right): \delta=6.53\left(\mathrm{~d}, J=6.5 \mathrm{~Hz}, 0.7 \mathrm{H}, \mathrm{N} H_{\beta}\right) ; 6.17\left(\mathrm{~d}, J=4.5 \mathrm{~Hz}, 0.3 \mathrm{H}, \mathrm{N} H_{\alpha}\right) ; 5.12(\mathrm{~d}, J=$ $\left.5.0 \mathrm{~Hz}, 0.7 \mathrm{H}, \mathrm{O} H_{\alpha}\right) ; 5.08\left(\mathrm{~d}, J=5.0 \mathrm{~Hz}, 0.3 \mathrm{H}, \mathrm{O} H_{\beta}\right) ; 5.05\left(\mathrm{~d}, J=4.5 \mathrm{~Hz}, 0.7 \mathrm{H}, \mathrm{O} H_{\alpha}\right) ; 5.03(\mathrm{~d}, J=$ $\left.6.0 \mathrm{~Hz}, 0.7 \mathrm{H}, \mathrm{O} H_{\alpha}\right) ; 4.98\left(\mathrm{~d}, J=4.5 \mathrm{~Hz}, 0.3 \mathrm{H}, \mathrm{O} H_{\beta}\right) ; 4.90\left(\mathrm{t}, J=5.0 \mathrm{~Hz}, 0.7 \mathrm{H}, \mathrm{H}-1^{\prime}{ }_{\alpha}\right) ; 4.80(\mathrm{~d}, J=$ $\left.7.0 \mathrm{~Hz}, 0.7 \mathrm{H}, \mathrm{OH}-2_{\alpha}\right) ; 4.78\left(\mathrm{~d}, J=4.5 \mathrm{~Hz}, 0.7 \mathrm{H}, \mathrm{O} H_{\alpha}\right) ; 4.72(\mathrm{~d}, J=5.5 \mathrm{~Hz}, 0.3 \mathrm{H}, \mathrm{OH}) ; 4.62(\mathrm{t}, J=$ $\left.6.0 \mathrm{~Hz}, 0.3 \mathrm{H}, \mathrm{OH}-6_{\beta}\right) ; 4.58\left(\mathrm{~d}, J=4.5 \mathrm{~Hz}, 0.3 \mathrm{H}, \mathrm{O} H_{\beta}\right) ; 4.49\left(\mathrm{~d}, J=5.5 \mathrm{~Hz}, 0.7 \mathrm{H}, \mathrm{O} H_{\alpha}\right) ; 4.47(\mathrm{~d}, J=$ $\left.5.5 \mathrm{~Hz}, 0.7 \mathrm{H}, \mathrm{O} H_{\alpha}\right) ; 4.44\left(\mathrm{t}, J=6.0 \mathrm{~Hz}, 0.7 \mathrm{H}, \mathrm{OH}-6_{\alpha}\right) ; 4.40\left(\mathrm{~d}, J=6.5 \mathrm{~Hz}, 0.7 \mathrm{H}, \mathrm{O} H_{\beta}\right) ; 4.32(\mathrm{t}, J=$ $\left.6.0 \mathrm{~Hz}, 0.3 \mathrm{H}, \mathrm{OH}-6_{\beta}\right) ; 4.27\left(\mathrm{dd}, J=7.5,6.5 \mathrm{~Hz}, 0.3 \mathrm{H}, \mathrm{H}-{ }^{\prime}{ }_{\beta}\right) ; 4.24\left(\mathrm{~d}, J=5.5 \mathrm{~Hz}, 0.3 \mathrm{H}, \mathrm{O} H_{\beta}\right) ; 4.21$ $\left(\mathrm{d}, J=9.5 \mathrm{~Hz}, 0.3 \mathrm{H}, \mathrm{H}-1_{\beta}\right) ; 4.05\left(\mathrm{~d}, J=9.5 \mathrm{~Hz}, 0.7 \mathrm{H}, \mathrm{H}-1_{\alpha}\right) ; 3.70-3.66\left(\mathrm{~m}, 1.3 \mathrm{H}, \mathrm{H}-6_{\alpha}, 2 \times \mathrm{H}-6_{\beta}\right)$; $3.58\left(\mathrm{ddd}, J=11.5,5.5,2.5 \mathrm{~Hz}, 0.3 \mathrm{H}, \mathrm{H}-6_{\beta}\right) ; 3.53\left(\mathrm{dd}, J=13.0,1.5 \mathrm{~Hz}, 0.7 \mathrm{H}, \mathrm{H}-6_{\alpha}\right) ; 3.48-3.40(\mathrm{~m}$, 2.7H, H-2 $\left.{ }_{\alpha}, \mathrm{H}-2_{\beta}, 2 \times \mathrm{H}-6_{\alpha}, \mathrm{H}-6_{\beta}\right) ; 3.26-3.18(\mathrm{~m}, 2.3 \mathrm{H}), 3.10-3.02(\mathrm{~m}, 4 \mathrm{H})\left(\mathrm{H}-2{ }_{\alpha}, \mathrm{H}-3_{\alpha}, \mathrm{H}-3_{\beta}, \mathrm{H}-\right.$ $\left.3{ }_{\alpha}, \mathrm{H}-3{ }_{\beta}, \mathrm{H}-4, \mathrm{\alpha}, \mathrm{H}-4_{\beta}, \mathrm{H}-4{ }_{\alpha}{ }_{\alpha}, \mathrm{H}-4{ }_{\beta}, \mathrm{H}-5_{\alpha}, \mathrm{H}-5_{\beta}, \mathrm{H}-5{ }_{\alpha}, \mathrm{H}-5{ }_{\beta}\right) ; 2.89$ (td, $J=9.0,4.5 \mathrm{~Hz}, 0.3 \mathrm{H}, \mathrm{H}-$ $\left.2{ }_{\beta}{ }_{\beta}\right)$, assignments were confirmed by ${ }^{1} \mathrm{H}-{ }^{1} \mathrm{H}$ gCOSY.${ }^{13} \mathrm{C}$ NMR $\left(125 \mathrm{MHz}, \mathrm{DMSO}-d_{6}\right): \delta=96.9(\mathrm{C}-$ $\left.1^{\prime}{ }_{\beta}\right) ; 92.2\left(\mathrm{C}-1{ }_{\alpha}\right) ; 90.5\left(\mathrm{C}-1_{\beta}\right) ; 84.6\left(\mathrm{C}-1_{\alpha}\right) ; 81.0\left(\mathrm{C}-5{ }_{\alpha}{ }_{\alpha}, \mathrm{C}-5{ }_{\beta}{ }_{\beta}\right) ; 78.4,76.8,76.7,73.1,72.4,70.6$, $70.5,69.8,69.6,\left(\mathrm{C}-2{ }^{\prime}{ }_{\alpha}, \mathrm{C}-3{ }_{\alpha}, \mathrm{C}-3_{\beta}, \mathrm{C}-3{ }^{\prime}{ }_{\alpha}, \mathrm{C}-3{ }^{\prime}{ }_{\beta}, \mathrm{C}-4_{\alpha}, \mathrm{C}-4_{\beta}, \mathrm{C}-4{ }_{\alpha}{ }_{\alpha}, \mathrm{C}-4{ }^{\prime}{ }_{\beta}\right) ; 77.3\left(\mathrm{C}-5_{\alpha}, \mathrm{C}-5_{\beta}\right) ; 74.8$ $\left(\mathrm{C}-2{ }^{\prime}{ }_{\beta}\right) ; 71.9\left(\mathrm{C}-2_{\beta}\right) ; 70.3\left(\mathrm{C}-2_{\alpha}\right) ; 61.2(2 \mathrm{C})\left(\mathrm{C}-6_{\alpha}, \mathrm{C}-6{ }_{\alpha}{ }_{\alpha}\right) ; 61.0\left(\mathrm{C}-6_{\beta}, \mathrm{C}-6{ }^{\prime}{ }_{\beta}\right)$, assignments were confirmed by ${ }^{1} \mathrm{H}-{ }^{13} \mathrm{C}$ HSQC. LRMS $\left(\mathrm{ESI}^{+}\right): m / z=428[\mathrm{M}+\mathrm{Na}]^{+}$. HRMS: Calcd for $\mathrm{C}_{12} \mathrm{H}_{23} \mathrm{NO}_{12} \mathrm{SNa} 428.0833$, Found 428.0832. 
2-Deoxy-2- $N$-(1-S- $\beta$-D-glucopyranosyl)-2-sulfonylamino- $\alpha, \beta$-D-glucopyranose (15c). The title compound 15c was obtained from compound $\mathbf{1 5 b}$ according to the general procedure 3 . The lyophilized compound 15c (87\% yield, $\alpha / \beta$ 70:30) was obtained as a clear oil. $\mathrm{R}_{f}=0.16(95: 5$ $\left.\mathrm{CH}_{3} \mathrm{CN} / \mathrm{H}_{2} \mathrm{O}\right) .{ }^{1} \mathrm{H}$ NMR $\left(500 \mathrm{MHz}, \mathrm{DMSO}-d_{6}\right): \delta=6.89\left(\mathrm{~d}, J=9.0 \mathrm{~Hz}, 0.3 \mathrm{H}, \mathrm{N} H_{\beta}\right) ; 6.73(\mathrm{~d}, J=$ $\left.5.5 \mathrm{~Hz}, 0.3 \mathrm{H}, \mathrm{OH}-1_{\beta}\right) ; 6.61\left(\mathrm{~d}, J=8.5 \mathrm{~Hz}, 0.7 \mathrm{H}, \mathrm{N} H_{\alpha}\right) ; 6.58\left(\mathrm{~d}, J=4.5 \mathrm{~Hz}, 0.7 \mathrm{H}, \mathrm{OH}-1_{\alpha}\right) ; 5.21(\mathrm{~d}, J$ $=5.5 \mathrm{~Hz}, 0.7 \mathrm{H}), 5.11(\mathrm{~d}, J=5.0 \mathrm{~Hz}, 0.7 \mathrm{H}), 5.10(\mathrm{~d}, J=4.0 \mathrm{~Hz}, 0.3 \mathrm{H}), 5.07(\mathrm{~d}, J=5.5 \mathrm{~Hz}, 0.7 \mathrm{H})$, $5.05(\mathrm{~d}, J=4.5 \mathrm{~Hz}, 0.3 \mathrm{H}), 5.04(\mathrm{~d}, J=6.5 \mathrm{~Hz}, 0.3 \mathrm{H})\left(3 \times \mathrm{OH}_{\alpha}, 3 \times \mathrm{OH}_{\beta}\right) ; 4.98-4.96(\mathrm{~m}, 1.7 \mathrm{H}, \mathrm{H}-$ $\left.1^{\prime}{ }_{\alpha}, \mathrm{OH}-6_{\alpha}, \mathrm{OH}{ }_{\beta}\right) ; 4.89(\mathrm{~d}, J=5.5 \mathrm{~Hz}, 0.7 \mathrm{H}), 4.86(\mathrm{~d}, J=6.0 \mathrm{~Hz}, 0.7 \mathrm{H})\left(2 \times \mathrm{O} H_{\alpha}\right) ; 4.52-4.48(\mathrm{~m}$, $\left.0.6 \mathrm{H}, 2 \times \mathrm{OH}_{\beta}\right) ; 4.42-4.37\left(\mathrm{~m}, 1.3 \mathrm{H}, \mathrm{H}-{ }_{1}{ }_{\beta}, \mathrm{H}-1_{\beta}, \mathrm{OH}-6_{\alpha}\right) ; 4.30\left(\mathrm{~m}, 0.3 \mathrm{H}, \mathrm{OH}-6_{\beta}\right) ; 4.29(\mathrm{~d}, J=9.5$ $\left.\mathrm{Hz}, 0.7 \mathrm{H}, \mathrm{H}-1_{\alpha}\right) ; 3.74\left(\mathrm{~m}, 0.7 \mathrm{H}, \mathrm{H}-6_{\alpha}\right) ; 3.68\left(\mathrm{~m}, 0.3 \mathrm{H}, \mathrm{H}-6_{\beta}\right) ; 3.60\left(\mathrm{~m}, 1 \mathrm{H}, \mathrm{H}-6_{\alpha, \beta}\right) ; 3.55$ (m, 0.7H, H-5 $)$ ) 3.51-3.42 (m, 3.6H, H- ${ }_{\alpha, \beta}$, H- $5_{\alpha}, 2 \times$ H- $5_{\beta}, H-6_{\alpha}, 2 \times$ H- $\left.6_{\beta}\right) ; 3.26-3.23$ (m, 2.1H), 3.13-3.00 $(\mathrm{m}, 2.3 \mathrm{H})\left(\mathrm{H}-3_{\alpha, \beta}, \mathrm{H}-3{ }_{\alpha, \beta}, \mathrm{H}-4_{\alpha, \beta}, \mathrm{H}-4{ }_{\alpha, \beta}, \mathrm{H}-6_{\alpha}\right) ; 3.21$ (t, $\left.J=10.0 \mathrm{~Hz}, 0.3 \mathrm{H}, \mathrm{H}-3{ }^{\prime}{ }_{\beta}\right) ; 3.13(\mathrm{~m}, 0.7 \mathrm{H}$, $\left.\mathrm{H}-2{ }_{\alpha}{ }_{\alpha}\right) ; 2.87$ (q, $\left.J=9.0 \mathrm{~Hz}, 0.3 \mathrm{H}, \mathrm{H}-2{ }_{\beta}{ }_{\beta}\right)$, assignments were confirmed by ${ }^{1} \mathrm{H}-{ }^{1} \mathrm{H}$ gCOSY.${ }^{13} \mathrm{C}$ NMR $\left(125 \mathrm{MHz}, \mathrm{DMSO}-d_{6}\right): \delta=95.6\left(\mathrm{C}-1^{\prime}{ }_{\beta}\right) ; 91.9\left(\mathrm{C}-1_{\alpha}{ }_{\alpha}\right) ; 90.5\left(\mathrm{C}-1_{\alpha}\right) ; 89.5\left(\mathrm{C}-1_{\beta}\right) ; 81.5,80.7,77.9$, 77.2, 76.5, 74.5, 72.0, 70.9 (2C), 70.8, 70.1, 69.6 (C-2 ${ }_{\alpha, \beta}, \mathrm{C}-3_{\alpha, \beta}, \mathrm{C}-3_{\alpha, \beta}{ }_{\alpha, \beta}, \mathrm{C}-4_{\alpha, \beta}, \mathrm{C}-4{ }_{\alpha, \beta}{ }_{\alpha, \mathrm{C}} \mathrm{C}-5_{\alpha, \beta}, \mathrm{C}-$ $\left.{ }^{\prime}{ }_{\alpha, \beta}\right) ; 61.7\left(\mathrm{C}-2{ }_{\beta}{ }_{\beta}\right) ; 61.2,61.0\left(\mathrm{C}-6_{\alpha, \beta}, \mathrm{C}-6{ }^{\prime}{ }_{\alpha, \beta}\right) ; 58.7\left(\mathrm{C}-2{ }^{\prime}{ }_{\alpha}\right)$, assignments were confirmed by ${ }^{1} \mathrm{H}^{-13} \mathrm{C}$ HSQC. LRMS $\left(\mathrm{ESI}^{+}\right): m / z=428[\mathrm{M}+\mathrm{Na}]^{+}$. HRMS: Calcd for $\mathrm{C}_{12} \mathrm{H}_{23} \mathrm{NO}_{12} \mathrm{SNa} 428.0833$, Found 428.0812 .

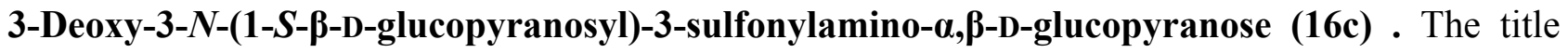
compound 16c was obtained from compound $\mathbf{1 6 b}$ according to the general procedure 3 . The lyophilized compound was obtained as a colorless gum (84\% yield) as a mixture of $\alpha$ and $\beta$ anomers $\left(\alpha / \beta\right.$ 40:60). $\mathrm{R}_{f}=0.14\left(85: 15 \mathrm{CH}_{3} \mathrm{CN} / \mathrm{H}_{2} \mathrm{O}\right) .{ }^{1} \mathrm{H}$ NMR $\left(500 \mathrm{MHz}, \mathrm{DMSO}-d_{6}\right): \delta=7.04(\mathrm{~d}, J$ 
$\left.=7.0 \mathrm{~Hz}, 0.6 \mathrm{H}, \mathrm{N} H_{\beta}\right) ; 6.99\left(\mathrm{~d}, J=7.5 \mathrm{~Hz}, 0.4 \mathrm{H}, \mathrm{N} H_{\alpha}\right) ; 6.66\left(\mathrm{~d}, J=6.0 \mathrm{~Hz}, 0.6 \mathrm{H}, \mathrm{OH}-1_{\beta}\right) ; 6.41(\mathrm{~d}, J$ $\left.=4.0 \mathrm{~Hz}, 0.4 \mathrm{H}, \mathrm{OH}-1_{\alpha}\right) ; 5.09(\mathrm{br} \mathrm{s}, 2 \mathrm{H}), 5.03(\mathrm{~d}, J=4.0 \mathrm{~Hz}, 1 \mathrm{H})\left(3 \times \mathrm{OH}_{\alpha}, 3 \times \mathrm{O} H_{\beta}\right) ; 4.94(\mathrm{t}, J=$ $\left.3.5 \mathrm{~Hz}, 0.4 \mathrm{H}, \mathrm{H}-{ }^{\prime}{ }_{\alpha}\right) ; 4.91\left(\mathrm{~d}, J=5.5 \mathrm{~Hz}, 0.6 \mathrm{H}, \mathrm{OH}-2^{\prime}{ }_{\beta}\right) ; 4.75\left(\mathrm{~d}, J=6.5 \mathrm{~Hz}, 0.6 \mathrm{H}, \mathrm{O} H_{\beta}\right) ; 4.70-4.67$ (m, 1.4H, $\left.\mathrm{OH}-6_{\beta}, 1 \times \mathrm{OH}_{\alpha}, 1 \times \mathrm{OH}_{\beta}\right) ; 4.52\left(\mathrm{t}, J=5.5 \mathrm{~Hz}, 0.6 \mathrm{H}, \mathrm{O} H-6_{\beta}\right) ; 4.48(\mathrm{~d}, J=7.5 \mathrm{~Hz}, 0.4 \mathrm{H}, 1$ $\left.\times \mathrm{OH}_{\alpha}\right) ; 4.41\left(\mathrm{~m}, 0.4 \mathrm{H}, 1 \times \mathrm{OH}_{\alpha}\right) ; 4.40\left(\mathrm{~d}, J=9.5 \mathrm{~Hz}, 0.6 \mathrm{H}, \mathrm{H}-1{ }^{\prime}\right) ; 4.39(\mathrm{~d}, J=9.5 \mathrm{~Hz}, 0.4 \mathrm{H}, \mathrm{H}-$ $\left.1{ }_{\alpha}^{\prime}\right) ; 4.30$ (br t, $\left.J=6.0 \mathrm{~Hz}, 0.4 \mathrm{H}, \mathrm{H}-1{ }^{\prime}{ }_{\beta}\right) ; 3.72-3.69\left(\mathrm{~m}, 1 \mathrm{H}, \mathrm{H}-6_{\alpha}, \mathrm{H}-6_{\beta}\right) ; 6.65(\mathrm{dd}, J=11.5,3.0 \mathrm{~Hz}$, 0.4H, H-6 $\left.6_{\alpha}\right) ; 3.57$ (m, 0.6H, H- $\left.6_{\beta}\right) ; 3.46-3.38$ (m, $\left.3 \mathrm{H}, \mathrm{H}-2_{\alpha}, \mathrm{H}-2_{\beta}, 2 \times \mathrm{H}-6_{\alpha}, 2 \times \mathrm{H}-6_{\beta}\right) ; 3.22(\mathrm{~m}$, 0.4H, H-2 $\left.{ }_{\alpha}\right)$; 3.21-3.03 (m, 5.4H, H-2 ${ }_{\alpha}, \mathrm{H}-3_{\alpha}, \mathrm{H}-3_{\beta}, \mathrm{H}-4_{\alpha}, \mathrm{H}-4_{\beta}, \mathrm{H}-4{ }_{\alpha}{ }_{\alpha}, \mathrm{H}-4{ }^{\prime}, \mathrm{H}-5_{\alpha}, \mathrm{H}-5_{\beta}, \mathrm{H}-5{ }_{\alpha}, \mathrm{H}-$ $\left.{ }^{5}{ }_{\beta}\right) ; 3.01\left(\mathrm{~m}, 1 \mathrm{H}, \mathrm{H}-3{ }_{\alpha}{ }_{\alpha}, \mathrm{H}-3{ }^{\prime}{ }\right)$; $2.99-2.97$ (m, 0.6H, H-2 ${ }_{\beta}$ ), assignments were confirmed by ${ }^{1} \mathrm{H}-{ }^{1} \mathrm{H}$ gCOSY. ${ }^{13} \mathrm{C}$ NMR $\left(125 \mathrm{MHz}, \mathrm{DMSO}-d_{6}\right): \delta=97.4\left(\mathrm{C}-1_{\beta}^{\prime}\right) ; 91.7\left(\mathrm{C}-1_{\alpha}^{\prime}{ }_{\alpha}\right) ; 89.6\left(\mathrm{C}-1_{\beta}\right) ; 89.4\left(\mathrm{C}-1_{\alpha}\right)$; $81.1\left(\mathrm{C}-5^{\prime}{ }_{\alpha}\right) ; 81.0\left(\mathrm{C}-5^{\prime}{ }_{\beta}\right) ; 77.9,77.8,77.6\left(\mathrm{C}-3_{\alpha}, \mathrm{C}-3_{\beta}, \mathrm{C}-5_{\alpha}, \mathrm{C}-5_{\beta}\right) ; 72.9\left(\mathrm{C}-2{ }^{\prime}{ }_{\beta}\right) ; 72.4\left(\mathrm{C}-2{ }_{\alpha}{ }_{\alpha}\right) ; 70.9$ $\left(\mathrm{C}-2_{\alpha}\right) ; 70.4\left(\mathrm{C}-2_{\beta}\right) ; 70.0\left(\mathrm{C}-4{ }^{\prime}{ }_{\beta}\right) ; 69.9\left(\mathrm{C}-4_{\alpha}{ }_{\alpha}\right) ; 69.1\left(\mathrm{C}-4_{\beta}\right) ; 68.9\left(\mathrm{C}-4_{\alpha}\right) ; 62.7\left(\mathrm{C}-3{ }^{\prime}{ }_{\beta}\right) ; 61.2,61.3(\mathrm{C}-$ $\left.6_{\alpha}, \mathrm{C}-6_{\beta}, \mathrm{C}-6^{\prime}{ }_{\alpha}, \mathrm{C}-6^{\prime}{ }_{\beta}\right) ; 59.1\left(\mathrm{C}-3^{\prime}{ }_{\alpha}\right)$, assignments were confirmed by ${ }^{1} \mathrm{H}-{ }^{13} \mathrm{C}$ HSQC. LRMS (ESI $\left.{ }^{+}\right)$: $m / z=428[\mathrm{M}+\mathrm{Na}]^{+}$. HRMS: Calcd for $\mathrm{C}_{12} \mathrm{H}_{23} \mathrm{NO}_{12} \mathrm{SNa} 428.0833$, Found 428.0817.

\section{Methyl 4-deoxy-4-N-(1-S- $\beta$-D-glucopyranosyl)-4-sulfonylamino- $\alpha-D-g l u c o p y r a n o s i d e \quad(17 c)$.}

The title compound $\mathbf{1 7} \mathbf{c}$ was obtained from compound $\mathbf{1 7 b}$ according to the general procedure 3 .

The lyophilized compound was obtained as a highly hygroscopic white solid ( $99 \%$ yield). $\mathrm{R}_{f}=0.04$ (8:2 $\left.\mathrm{CH}_{2} \mathrm{Cl}_{2} / \mathrm{MeOH}\right) \cdot[\alpha]^{25}{ }_{\mathrm{D}}=+67(c=1.0, \mathrm{MeOH}) .{ }^{1} \mathrm{H}$ NMR $\left(500 \mathrm{MHz}, \mathrm{DMSO}-d_{6}\right): \delta=7.23(\mathrm{~d}$, $J=9.0 \mathrm{~Hz}, 1 \mathrm{H}, \mathrm{N} H) ; 5.07$ (br s, 1H, OH-3); $5.05(\mathrm{~d}, J=6.0 \mathrm{~Hz}, 1 \mathrm{H}, \mathrm{OH}-2) ; 5.03$ (br d, $J=3.5 \mathrm{~Hz}$, 1H, $\mathrm{OH}-4) ; 4.85\left(\mathrm{~d}, J=5.0 \mathrm{~Hz}, 1 \mathrm{H}, \mathrm{OH}-2^{\prime}\right) ; 4.77$ (d, $J=5.5 \mathrm{~Hz}, 1 \mathrm{H}, \mathrm{OH}-3^{\prime}$ ); 4.69 (br t, $J=5.5 \mathrm{~Hz}$ 1H, $\mathrm{OH}-6$ or $\mathrm{OH}-6^{\prime}$ ); 4.54 (d, $J=3.5 \mathrm{~Hz}, 1 \mathrm{H}, \mathrm{H}-1^{\prime}$ ); 4.44 (br t, $J=5.0 \mathrm{~Hz}, 1 \mathrm{H}, \mathrm{OH}-6$ or $\left.\mathrm{O} H-6^{\prime}\right)$; 4.33 (d, $J=10.0 \mathrm{~Hz}, 1 \mathrm{H}, \mathrm{H}-1) ; 3.76-3.69$ (m, 2H, H-6a, H-6a'); 3.56 (t d, $J=9.0,5.5 \mathrm{~Hz}, 1 \mathrm{H}, \mathrm{H}-$ 
3'); 3.43 (t, $J=9.0 \mathrm{~Hz}, 1 \mathrm{H}, \mathrm{H}-2$ ); 3.42-3.37 (m, 3H, H-5', H-6b, H-6b'); 3.27 (s, 3H, OCH $)$ ); 3.233.16 (m, 3H, H-2', H-3, H-5'); 3.00 (br t, $J=8.0 \mathrm{~Hz}, 1 \mathrm{H}, \mathrm{H}-4) ; 2.88$ (q, $J=9.5 \mathrm{~Hz}, 1 \mathrm{H}, \mathrm{H}-4$ '), assignments were confirmed by ${ }^{1} \mathrm{H}-{ }^{1} \mathrm{H}$ gCOSY.${ }^{13} \mathrm{C}$ NMR (125 MHz, DMSO- $\left.d_{6}\right): \delta=99.3(\mathrm{C}-1$ '); 88.8 (C-1); 80.8 (C-2'); 78.0 (C-3); 72.7 (C-5); 72.0 (C-5'); 71.1 (C-3’); 70.6 (C-2); 70.2 (C-4); 61.5, 61.3 (C-6, C-6'); 56.7 (C-4'); $54.4\left(\mathrm{OCH}_{3}\right)$, assignments were confirmed by ${ }^{1} \mathrm{H}^{-13} \mathrm{C}$ HSQC. LRMS (ESI $\left.{ }^{+}\right): m / z=437\left[\mathrm{M}+\mathrm{NH}_{4}\right]^{+}, 442[\mathrm{M}+\mathrm{Na}]^{+}$. HRMS: Calcd for $\mathrm{C}_{13} \mathrm{H}_{25} \mathrm{NO}_{12} \mathrm{SNa}$ 442.0990, Found 442.1009 .

\section{Methyl 6-deoxy-6- $N$-(1-S- $\beta$-D-glucopyranosyl)-6-sulfonylamino- $\alpha$-D-glucopyranoside (18c).}

The title compound $\mathbf{1 8 c}$ was obtained from compound $\mathbf{1 8 b}$ according to the general procedure 3. The lyophilized compound was obtained as a highly hygroscopic white solid ( $87 \%$ yield). $\mathrm{R}_{f}=0.15$ $\left(9: 1 \mathrm{CH}_{3} \mathrm{CN} / \mathrm{H}_{2} \mathrm{O}\right) \cdot[\alpha]^{25}=+49(c=1.0, \mathrm{MeOH}) .{ }^{1} \mathrm{H}$ NMR $\left(500 \mathrm{MHz}, \mathrm{DMSO}-d_{6}\right): \delta=6.78(\mathrm{br} \mathrm{s}$, 1H, NH); $5.11($ br s, 2H, OH-2, OH-2'); $5.04(\mathrm{~d}, J=4.5 \mathrm{~Hz}, 1 \mathrm{H}), 4.98(\mathrm{~d}, J=5.0 \mathrm{~Hz}, 1 \mathrm{H})(\mathrm{O} H-4$, $\left.\mathrm{O} H-4^{\prime}\right) ; 4.77(\mathrm{~d}, J=3.5 \mathrm{~Hz}, 1 \mathrm{H}), 4.71(\mathrm{~d}, J=6.5 \mathrm{~Hz}, 1 \mathrm{H})\left(\mathrm{OH}-3, \mathrm{OH}-3^{\prime}\right) ; 4.52(\mathrm{~d}, J=3.5 \mathrm{~Hz}, 1 \mathrm{H}$, H-1'); $4.47(\mathrm{t}, J=6.0 \mathrm{~Hz}, 1 \mathrm{H}, \mathrm{OH}-6) ; 4.20(\mathrm{~d}, J=9.5 \mathrm{~Hz}, 1 \mathrm{H}, \mathrm{H}-1) ; 3.69$ (dd, $J=11.5,4.5 \mathrm{~Hz}, 1 \mathrm{H}$, H-6a); 3.47 (dd, $J=11.5,7.0 \mathrm{~Hz}, 1 \mathrm{H}, \mathrm{H}-6 \mathrm{~b}) ; 3.46$ (t, $J=7.0 \mathrm{~Hz}, 1 \mathrm{H}, \mathrm{H}-2) ; 3.43$ (m, 1H, H-6a'); 3.40-3.35 (m, 2H, H-3, H-3'); 3.28 (s, 3H, OCH $)$; 3.25-3.21 (m, 2H, H-5, H-5'); 3.19 (m, 1H, H2'); 3.01 (td, $J=9.5,4.5 \mathrm{~Hz}, 1 \mathrm{H}, \mathrm{H}-4$ or H-4'); 3.05 (m, 1H, H-6b'); 2.98 (td, $J=9.0,4.0 \mathrm{~Hz}, 1 \mathrm{H}$, H-4' or H-4), assignments were confirmed by ${ }^{1} \mathrm{H}-{ }^{1} \mathrm{H}$ gCOSY. ${ }^{13} \mathrm{C}$ NMR $\left(125 \mathrm{MHz}\right.$, DMSO- $\left.d_{6}\right): \delta=$ 99.7 (C-1'); 89.6 (C-1); 81.1, 77.3 (C-5, C-5'); 73.0 (C-3 or C-3'); 71.9 (C-2'); 71.6 (C-4 or C-4'); 71.1 (C-3' or $\mathrm{C}-3)$; 70.6 (C-2); 69.4 (C-4' or C-4); 60.8 (C-6); $54.5\left(\mathrm{OCH}_{3}\right) ; 44.7$ (C-6'), assignments were confirmed by ${ }^{1} \mathrm{H}_{-}{ }^{13} \mathrm{C}$ HSQC. LRMS $\left(\mathrm{ESI}^{+}\right): m / z=437\left[\mathrm{M}+\mathrm{NH}_{4}\right]^{+}, 442[\mathrm{M}+$ $\mathrm{Na}]^{+}$. HRMS: Calcd for $\mathrm{C}_{13} \mathrm{H}_{25} \mathrm{NO}_{12} \mathrm{SNa} 442.0990$, Found 442.0985 . 
Compound 19a was obtained from compound $\mathbf{2}$ and the glucosamine hydrochloride salt 7 according to the general procedure 1 . Purification by flash chromatography (1:1 acetone/hexane) afforded 19a as a mixture of $\alpha$ and $\beta$ anomers (68\% yield; $\alpha / \beta$ 70:30) as a light yellow solid. $\mathrm{R}_{f}=0.22(1: 1$ acetone/hexane). m.p. $=111-113{ }^{\circ} \mathrm{C} .{ }^{1} \mathrm{H}$ NMR $\left(500 \mathrm{MHz}, \mathrm{DMSO}-d_{6}, \beta\right.$-anomer $): \delta=6.28(\mathrm{~d}, J=$ $4.5 \mathrm{~Hz}, 1 \mathrm{H}, \mathrm{OH}-1) ; 5.25$ (m, 1H, H-3); 5.23 (m, 1H, H-4'); 5.18 (dd, J=10.0, 3.5 Hz, 1H, H-3’); 5.20 (d, $J=3.5 \mathrm{~Hz}, 1 \mathrm{H}, \mathrm{H}-1$ '); 4.99 (t, $J=9.5 \mathrm{~Hz}, 1 \mathrm{H}, \mathrm{H}-2)$; 4.85 (dd, $\left.J=10.0,8.0 \mathrm{~Hz}, 1 \mathrm{H}, \mathrm{H}-2^{\prime}\right)$; $4.77(\mathrm{~d}, J=4.5 \mathrm{~Hz}, 1 \mathrm{H}, \mathrm{OH}-4$ '); 4.70 (d, $J=8.0 \mathrm{~Hz}, 1 \mathrm{H}, \mathrm{H}-1$ '); 4.64 (d, $J=4.5 \mathrm{~Hz}, 1 \mathrm{H}, \mathrm{OH}-3$ ”); 4.34 (d, $J=9.5 \mathrm{~Hz}, 1 \mathrm{H}, \mathrm{H}-1)$; 4.31 (br d, $J=12.0 \mathrm{~Hz}, 1 \mathrm{H}, \mathrm{H}-6 \mathrm{a}$ '); 4.28 (t, $J=5.5 \mathrm{~Hz}, 1 \mathrm{H}, \mathrm{OH}-6$ ”); 4.22 (br t, $J=12.0$ Hz, 1H, H-5'); 4.05-3.99 (m, 3H, H-6a, H-6b, H-6b'); 3.38-3.31 (m, 2H, H-4, H5); 3.60 (dd, $J=11.0,4.5$ Hz, 1H, H-6a”); 5.44 (m, 1H, H-5”); 3.46 (dd, $J=11.0,5.5$ Hz, 1H, H$6 \mathrm{~b}$ ”); 3.26 (td, $J=10.0,5.0 \mathrm{~Hz}, 1 \mathrm{H}, \mathrm{H}-3$ ”); 3.03 (td, $J=9.5,4.5 \mathrm{~Hz}, 1 \mathrm{H}, \mathrm{H}-4$ "); 2.97 (d, $J=8.0 \mathrm{~Hz}$, $1 \mathrm{H}, \mathrm{NH}) ; 2.44(\mathrm{td}, J=9.0,3.0 \mathrm{~Hz}, 1 \mathrm{H}, \mathrm{H}-2$ ”) $) 2.12,2.11,2.01,2.00,1.90\left(5 \times \mathrm{s}, 21 \mathrm{H}, \mathrm{OCOCH}_{3}\right)$, assignments were confirmed by ${ }^{1} \mathrm{H}^{-1} \mathrm{H}$ gCOSY. ${ }^{13} \mathrm{C}$ NMR $\left(125 \mathrm{MHz}, \mathrm{DMSO}-d_{6}\right): \delta=170.6,169.8$ (2C), 169.5, 169.4, 169.3, $169.0\left(\mathrm{OCOCH}_{3}\right) ; 99.9(\mathrm{C}-1$ '); $91.5(\mathrm{C}-1$ ”’); 86.6 (C-1); 75.7, 75.5 (C-4, C-5); 73.1 (C-3); 72.7 (C-3”); 71.8 (C-5’); 70.9 (C-4’); 70.3 (C-3’); 69.5 (C-5’); 68.9 (C-2'); 68.6 (C-2”); 67.6 (C-2); 66.9 (C-4’); 62.2 (C-6’); 61.3 (C-6”); 60.6 (C-6); 20.7, 20.6 (2C), 20.5, 20.4, 20.3 (2C) $\left(\mathrm{OCOCH}_{3}\right)$, assignments were confirmed by ${ }^{1} \mathrm{H}-{ }^{13} \mathrm{C}$ HSQC. LRMS $\left(\mathrm{ESI}^{+}\right): \mathrm{m} / z=852$ [M $+\mathrm{Na}]^{+}$. HRMS: Calcd for $\mathrm{C}_{32} \mathrm{H}_{47} \mathrm{NO}_{22} \mathrm{SNa}$ 852.2203, Found 852.2202.

\section{Methyl 6-deoxy-6- $N$-(2,2',3,3',4',6,6'-hepta- $O$-acetyl-1-S- $\beta$-D-lactosyl)- $\alpha$-D-glucopyranoside} (20a). Compound 20a was obtained from compound 2 and $\mathbf{1 0}$ according to the general procedure 1. 
Purification by flash chromatography $\left(95: 5 \mathrm{CH}_{2} \mathrm{Cl}_{2} / \mathrm{MeOH}\right)$ afforded $20 \mathrm{a}(80 \%$ purity, $84 \%$ yield) as a white solid. $\mathrm{R}_{f}=0.25\left(1: 1\right.$ acetone/hexane). $\operatorname{LRMS}\left(\mathrm{ESI}^{+}\right): m / z=866[\mathrm{M}+\mathrm{Na}]^{+}$. HRMS: Calcd for $\mathrm{C}_{33} \mathrm{H}_{49} \mathrm{NO}_{22} \mathrm{SNa} 866.2359$, Found 866.2399. Compound 20a was used as reagent for general procedure 2 without further purification.

\section{Methyl 6-deoxy-6- $N$-(2,2',3,3',4',6,6'-hepta- $O$-acetyl-1-S- $\beta$-D-maltosyl)- $\alpha$-D-glucopyranoside} (21a). Compound 21a was obtained from compound $\mathbf{3}$ and $\mathbf{1 0}$ according to the general procedure 1. Purification by flash chromatography (2:1 acetone/hexane) afforded 21a (75\% yield) as a white solid. $\mathrm{R}_{f}=0.22\left(1: 1\right.$ acetone/hexane). m.p. $=123{ }^{\circ} \mathrm{C} .[\alpha]^{25}=+54\left(c=1.0\right.$, chloroform) ${ }^{1} \mathrm{H}$ NMR (500 MHz, DMSO- $\left.d_{6}\right): \delta=5.38$ (t, $\left.J=9.5 \mathrm{~Hz}, 1 \mathrm{H}, \mathrm{H}-3\right) ; 5.29$ (d, $J=3.5 \mathrm{~Hz}, 1 \mathrm{H}, \mathrm{H}-1$ '); 5.22 (t, $J=$ $\left.10.0 \mathrm{~Hz}, 1 \mathrm{H}, \mathrm{H}-3^{\prime}\right) ; 4.98$ (t, $J=10.0 \mathrm{~Hz}, 1 \mathrm{H}, \mathrm{H}-4^{\prime}$ ); 4.87 (d, $J=4.0 \mathrm{~Hz}, 1 \mathrm{H}, \mathrm{OH}-4$ '); 4.86 (dd, $J=$ 10.0, 3.5 Hz, 1H, H-2'); 4.83 (t, $J=9.5 \mathrm{~Hz}, 1 \mathrm{H}, \mathrm{H}-2) ; 4.71$ (d, $J=4.5 \mathrm{~Hz}, 1 \mathrm{H}, \mathrm{OH}-3$ ”); 4.67 (d, $J=$ $8.5 \mathrm{~Hz}, 1 \mathrm{H}, \mathrm{OH}-2 ") ; 4.65$ (d, $J=10.0 \mathrm{~Hz}, 1 \mathrm{H}, \mathrm{H}-1) ; 4.50$ (d, $J=3.5 \mathrm{~Hz}, 1 \mathrm{H}, \mathrm{H}-1$ "); 4.41 (dd, $J=$ 13.0, 3.0 Hz, 1H, H-6a); 4.16 (dd, $J=12.5,4.5 \mathrm{~Hz}, 1 \mathrm{H}, \mathrm{H}-6 \mathrm{a}$ '); 4.12 (dd, $J=12.0,5.0 \mathrm{~Hz}, 1 \mathrm{H}, \mathrm{H}-$ 6b); 4.01 (dd, $\left.J=12.5,2.0 \mathrm{~Hz}, 1 \mathrm{H}, \mathrm{H}-6 \mathrm{~b}^{\prime}\right) ; 3.97$ (m, 1H, H-5'); 3.94 (m, 1H, H-5); 3.87 (t, $J=9.5$ Hz, 1H, H-4); 3.46 (t, $J=6.0 \mathrm{~Hz}, 1 \mathrm{H}, \mathrm{NH}) ; 3.35$ (m, 2H, H-3", H-5”); 3.28 (s, 3H, $\mathrm{OCH}_{3}$ ); 3.183.16 (m, 2H, H-2", H6a"); 2.95-2.89 (m, 2H, H-4", H-6b"); 2.07, 2.02, 1.99, 1.98, 1.97, 1.95 (6 × s, $\left.21 \mathrm{H}, \mathrm{OCOCH}_{3}\right)$, assignments were confirmed by ${ }^{1} \mathrm{H}-{ }^{1} \mathrm{H}$ gCOSY. ${ }^{13} \mathrm{C}$ NMR $\left(125 \mathrm{MHz}\right.$, DMSO- $\left.d_{6}\right)$ : $\delta=170.2,170.1,170.0,169.7(2 \mathrm{C}), 169.6,169.3\left(\mathrm{OCOCH}_{3}\right) ; 99.8\left(\mathrm{C}-1\right.$ '”); $95.6\left(\mathrm{C}-1{ }^{\prime}\right) ; 87.2(\mathrm{C}-1)$; 75.4 (C-3); 75.0 (C-5); 73.8 (C-4); 73.3 (C-3”); 72.0 (C-2”); 71.8 (C-4”); 71.2 (C-5”); 69.6 (C-2'); 69.0 (C-3’); 68.2 (C-2); 68.1 (C-5’); 67.9 (C-4'); 63.1 (C-6); 61.5 (C-6’); $55.5\left(\mathrm{C}-6\right.$ '); $54.6\left(\mathrm{OCH}_{3}\right)$; 20.7, 20.6, 20.5 (2C), 20.4 (2C), $20.3\left(\mathrm{OCOCH}_{3}\right)$, assignments were confirmed by ${ }^{1} \mathrm{H}-{ }^{13} \mathrm{C}$ HSQC. 
LRMS $\left(\mathrm{ESI}^{+}\right): m / z=844[\mathrm{M}+\mathrm{H}]^{+} ; 866[\mathrm{M}+\mathrm{Na}]^{+}$. HRMS: Calcd for $\mathrm{C}_{33} \mathrm{H}_{49} \mathrm{NO}_{22} \mathrm{SNa}$ 866.2359, Found 866.2332.

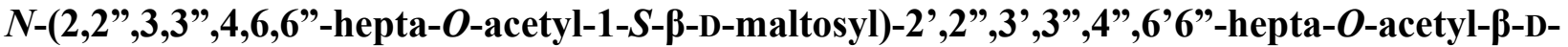

maltosamine (22a). Compound 22a was obtained from compound 3 and 11 according to the general procedure 1. Purification by flash chromatography (2:1 EtOAc/hexane) afforded 22a $(23 \%$ yield) as a white solid. $\mathrm{R}_{f}=0.31(2: 1 \mathrm{EtOAc} /$ hexane $) .{ }^{1} \mathrm{H}$ NMR $\left(500 \mathrm{MHz}, \mathrm{DMSO}-d_{6}\right): \delta=5.36(\mathrm{t}$, $J=9.0 \mathrm{~Hz}, 1 \mathrm{H}, \mathrm{H}-3) ; 5.30$ (d, $J=3.5 \mathrm{~Hz}, 2 \mathrm{H}, \mathrm{H}-1$ '); 5.23 (td, $J=10.0,2.0 \mathrm{~Hz}, 2 \mathrm{H}, \mathrm{H}-3$ ”); 5.21 (m, 1H, H-3'); 4.99 (td, $J=10.0,3.5 \mathrm{~Hz}, 2 \mathrm{H}, \mathrm{H}-4$ ”); 4.92 (br d, $J=9.2 \mathrm{~Hz}, 1 \mathrm{H}, \mathrm{NH}) ; 4.86$ (dd, $J=10.5$, $4.0 \mathrm{~Hz}, 2 \mathrm{H}, \mathrm{H}-2 ”) ; 4.84$ (t, $J=9.5 \mathrm{~Hz}, 1 \mathrm{H}, \mathrm{H}-2) ; 4.72$ (t, $J=9.0 \mathrm{~Hz}, 1 \mathrm{H}, \mathrm{H}-2$ '); 4.65 (d, $J=10.0$ Hz, 1H, H-1); 4.47 (dd, $J=12.5,1.5$ Hz, 1H, H-6a'); 4.41 (br d, $J=12.0 \mathrm{~Hz}, 1 \mathrm{H}, \mathrm{H}-6 \mathrm{a}) ; 4.34$ (t, $J=$ $9.0 \mathrm{~Hz}, 1 \mathrm{H}, \mathrm{H}-1$ '); 4.14 (br dd, $J=12.5,4.0 \mathrm{~Hz}, 4 \mathrm{H}, \mathrm{H}-6 \mathrm{~b}, \mathrm{H}-6 \mathrm{~b}$ ', H-6a”); 4.03 (br d, $J=12.0 \mathrm{~Hz}$, H-6b”); 3.98 (m, 2H, H-5”); 3.93 (m, 1H, H-5'); 3.83 (m, 3H, H-4, H-5, H-4'); 2.14, 2.10, 2.03, 2.01, 1.99, 1.98, 1.96, 1.94, $1.93\left(10 \times \mathrm{s}, 42 \mathrm{H}, \mathrm{OCOC} H_{3}\right)$, assignments were confirmed by ${ }^{1} \mathrm{H}-{ }^{1} \mathrm{H}$ gCOSY. ${ }^{13} \mathrm{C}$ NMR (125 MHz, DMSO-d $): \delta=170.7,170.6,170.4$ (2C), 170.3 (2C), 170.1, 170.0, 169.9 (2C), $169.6(3 \mathrm{C}) ; 169.5\left(\mathrm{OCOCH}_{3}\right) ; 95.9,95.8$ (C-1’); 91.2 (C-1'); 87.7 (C-1); 76.0 (C-3); 75.9 (C-3’); 75.4 (C-5’); 74.1, 73.9, 72.7 (C-4, C-5, C-4'); 71.6 (C-2’); 70.0, 69.9 (C-2’); 69.4 (2C) (C-3”); 68.5, 68.4 (C-5”); 68.4 (C-2); 68.2 (2C) (C-4”); 63.4 (C-6’); 63.3 (C-6); 61.9 (2C) (C-6”); $21.1(2 \mathrm{C}), 21.0(2 \mathrm{C}), 20.9(5 \mathrm{C}) ; 20.8(2 \mathrm{C}), 20.7$ (3C) $\left(\mathrm{OCOCH}_{3}\right)$, assignments were confirmed by ${ }^{1} \mathrm{H}^{13} \mathrm{C}$ HSQC. LRMS $\left(\mathrm{ESI}^{+}\right): m / z=1287[\mathrm{M}+\mathrm{H}]^{+} ; 1309[\mathrm{M}+\mathrm{Na}]^{+}$. HRMS: Calcd for $\mathrm{C}_{52} \mathrm{H}_{71} \mathrm{NO}_{34} \mathrm{SNa}$ 1308.3470, Found 1308.3427. 
Methyl 6-deoxy-6- $N$-([(2,3,4-tri- $O$-acetyl- $\alpha$-D-xylopyranosyl)-(1 $\rightarrow 6)]-(2,3,4-$ tri- $O$-acetyl- $\beta$-Dglucopyranosyl)-(1 $\rightarrow$ 4)-[(2,3,4-tri- $O$-acetyl- $\alpha$-D-xylopyranosyl)-(1 $\rightarrow 6)]-(2,3-$ di- $O$-acetyl- $\beta$-Dglucopyranosyl)-(1 $\rightarrow$ 4)-[(2,3,4-tri- $O$-acetyl- $\alpha$-D-xylopyranosyl)-(1 $\rightarrow$ 6)]-(2,3-di-O-acetyl- $\beta$-Dglucopyranosyl)-(1 $\rightarrow$ 4)-1,2,3,6-tetra- $O$-acetyl-1-S- $\beta$-D-glucopyranosyl)- $\alpha$-D-glucopyranoside (23a) - The title compound 23a was prepared from thioacetate $\mathbf{4}$ and amine $\mathbf{1 0}$ according to the general procedure 1 . Purification by flash chromatography $(2: 1$ acetone/hexane) afforded $23 \mathbf{a}(60 \%$ yield) as a white solid. $\mathrm{R}_{f}=0.19\left(1: 1\right.$ acetone/hexane). ${ }^{1} \mathrm{H}$ NMR $\left(500 \mathrm{MHz}, \mathrm{DMSO}-d_{6}\right): \delta=5.33(\mathrm{t}$, $J=9.5 \mathrm{~Hz}, 1 \mathrm{H}) ; 5.30(\mathrm{t}, J=9.5 \mathrm{~Hz}, 1 \mathrm{H}) ; 5.25(\mathrm{t}, J=9.5 \mathrm{~Hz}, 1 \mathrm{H}) ; 5.18(\mathrm{t}, J=10.0 \mathrm{~Hz}, 1 \mathrm{H}) ; 5.17(\mathrm{t}$, $J=9.5 \mathrm{~Hz}, 1 \mathrm{H}) ; 5.13-5.07(\mathrm{~m}, 3 \mathrm{H}) ; 5.00-4.71(\mathrm{~m}, 14 \mathrm{H}) ; 4.68-4.61(\mathrm{~m}, 3 \mathrm{H}) ; 4.55-4.48(\mathrm{~m}, 3 \mathrm{H}) ; 4.30$ $(\mathrm{m}, 1 \mathrm{H}) ; 4.14(\mathrm{~m}, 1 \mathrm{H}, \mathrm{NH}) ; 4.00(\mathrm{~m}, 1 \mathrm{H}) ; 3.93-3.59(\mathrm{~m}, 17 \mathrm{H}) ; 3.52-3.46(\mathrm{~m}, 2 \mathrm{H}) ; 3.36-3.32(\mathrm{~m}$, $2 \mathrm{H}) ; 3.27\left(\mathrm{~s}, 3 \mathrm{H}, \mathrm{OCH}_{3}\right) ; 3.17-3.13(\mathrm{~m}, 3 \mathrm{H}) ; 2.95-2.87(\mathrm{~m}, 2 \mathrm{H}) ; 2.09,2.08,2.06,2.05,2.04,2.01$, 2.00, 1.99, 1.97, 1.96, 1.95, $1.92\left(15 \times \mathrm{s}, 57 \mathrm{H}, \mathrm{OCOCH}_{3}\right) . \mathrm{LRMS}\left(\mathrm{ESI}^{+}\right): m / z=2070\left[\mathrm{M}+\mathrm{H}^{+}\right.$; $2092[\mathrm{M}+\mathrm{Na}]^{+}$. HRMS: Calcd for $\mathrm{C}_{84} \mathrm{H}_{117} \mathrm{NO}_{56} \mathrm{SNa} 2090.5951$, Found 2090.5989.

\section{2-Deoxy-2- $N$-(2,2',3,3',4',6,6'-hepta- $O$-acetyl-1-S- $\beta$-D-lactosyl)-2-sulfonylamino- $\alpha, \beta$-D-}

glucopyranose (19b). Compound 19b was obtained from compound 19a according to the general procedure 2. Purification by flash chromatography $\left(95: 5 \mathrm{CH}_{2} \mathrm{Cl}_{2} / \mathrm{MeOH}\right)$ afforded $\mathbf{1 9 b}(53 \%$ yield, $\beta$-anomer) as a white solid. $\mathrm{R}_{f}=0.32\left(9: 1 \mathrm{CH}_{2} \mathrm{Cl}_{2} / \mathrm{MeOH}\right)$. m.p. $=135-136{ }^{\circ} \mathrm{C} .{ }^{1} \mathrm{H} \mathrm{NMR}(500 \mathrm{MHz}$, DMSO- $\left.d_{6}\right): \delta=5.23($ br d, $J=3.5 \mathrm{~Hz}, 1 \mathrm{H}, \mathrm{H}-4$ '); 5.20 (t, $J=9.0 \mathrm{~Hz}, 1 \mathrm{H}, \mathrm{H}-3) ; 5.16(\mathrm{dd}, J=10.5$, $3.5 \mathrm{~Hz}, 1 \mathrm{H}, \mathrm{H}-3$ '); 4.89 (d, $J=5.0 \mathrm{~Hz}, 1 \mathrm{H}, \mathrm{OH}-3$ '); 4.85 (t, $\left.J=10.0 \mathrm{~Hz}, 2 \mathrm{H}, \mathrm{H}-2, \mathrm{H}-2^{\prime}\right) ; 4.76$ (d, $J$ $\left.=8.0 \mathrm{~Hz}, 1 \mathrm{H}, \mathrm{H}-1^{\prime}\right) ; 4.73(\mathrm{~d}, J=3.5 \mathrm{~Hz}, 1 \mathrm{H}, \mathrm{OH}-4$ ”); $4.96(\mathrm{~d}, J=6.05 \mathrm{~Hz}, 1 \mathrm{H}, \mathrm{NH}) ; 4.62(\mathrm{~d}, J=$ $10.0 \mathrm{~Hz}, 1 \mathrm{H}, \mathrm{H}-1) ; 4.51$ (d, $J=3.5 \mathrm{~Hz}, 1 \mathrm{H}, \mathrm{H}-1$ ”); 4.34 (br d, $J=11.5 \mathrm{~Hz}, 1 \mathrm{H}, \mathrm{H}-6 \mathrm{a}) ; 4.23$ (br t, $J=$ 7.0 Hz, 1H, H-5'); 4.03-3.99 (m, 3H, H6a', H-6b, H-6b'); 3.82 (m, 1H, H-5); 3.74 (t, J = 9.5 Hz, 
1H, H-4); 3.46 (t, $J=6.0$ Hz, 1H, OH-6"); 3.38-3.33 (m, 2H, H-4", H-5"); 3.21-3.15 (m, 3H, H-2", H-6a", OH-1"), 3.962.91(m, 2H, H-3", H-6b"); 2.11, 2.08, 2.01, 2.00, 1.99, 1.98, 1. (7 × s, 21H, $\mathrm{OCOCH}_{3}$ ), assignments were confirmed by ${ }^{1} \mathrm{H}-{ }^{1} \mathrm{H}$ gCOSY. ${ }^{13} \mathrm{C}$ NMR $\left(125 \mathrm{MHz}\right.$, DMSO- $\left.d_{6}\right): \delta=$ 170.2, 169.8, 169.6, 169.5, 169.4, 169.3, $169.0\left(\mathrm{OCOCH}_{3}\right) ; 99.9$ (C-1'); 99.7 (C-1"); $87.4(\mathrm{C}-1)$; 76.1 (C-4); 75.4 (C-5); 73.4 (C-3); 73.2 (C-5”); 71.9 (C-2”); 71.7 (C-3”); 71.1 (C-4”); 70.3 (C-3'); 69.7 (C-5'); 68.9, 67.8 (C-2, C-2'); 67.1 (C-4'); 62.4 (C-6); 60.8 (C-6’); 55.3 (C-6”); 20.6, 20.5, 20.4 (2C), 20.3 (2C), $20.2\left(\mathrm{OCOCH}_{3}\right)$, assignments were confirmed by ${ }^{1} \mathrm{H}^{13}{ }^{13} \mathrm{C}$ HSQC. LRMS $\left(\mathrm{ESI}^{+}\right): m / z=879\left[\mathrm{M}+\mathrm{NH}_{4}\right]^{+}, 884[\mathrm{M}+\mathrm{Na}]^{+}$. HRMS: Calcd for $\mathrm{C}_{32} \mathrm{H}_{47} \mathrm{NO}_{24} \mathrm{SNa}$ 884.2101, Found 884.2113.

\section{Methyl 6-deoxy-6- $N-\left(2,2^{\prime}, 3,3^{\prime}, 4^{\prime}, 6,6^{\prime}\right.$ 'hepta- $O$-acetyl-1- $S$ - $\beta$-D-lactosyl)-6-sulfonylamino- $\alpha$-D-}

glucopyranoside (20b). Compound 20b was obtained from compound 20a according to the general procedure 2. Purification by flash chromatography (3:2 acetone/hexane) afforded $20 \mathbf{b}$ ( $72 \%$ yield) as a white solid. $\mathrm{R}_{f}=0.16\left(3: 2\right.$ acetone/hexane). m.p. $=114-115{ }^{\circ} \mathrm{C} \cdot[\alpha]^{25} \mathrm{D}=+15(c=1.0$, chloroform). ${ }^{1} \mathrm{H}$ NMR (500 MHz, DMSO- $\left.d_{6}\right): \delta=7.45($ br s, $1 \mathrm{H}, \mathrm{NH}) ; 5.29(\mathrm{t}, J=9.5 \mathrm{~Hz}, 1 \mathrm{H}, \mathrm{H}-$ 3); 5.24 (d, $\left.J=3.0 \mathrm{~Hz}, 1 \mathrm{H}, \mathrm{H}-4^{\prime}\right) ; 5.16$ (dd, $J=10.5,4.5 \mathrm{~Hz}, 1 \mathrm{H}, \mathrm{H}-3$ '); 5.15 (t, $J=9.5 \mathrm{~Hz}, 1 \mathrm{H}, \mathrm{H}-$ 2); 4.97 (d, $J=5.5 \mathrm{~Hz}, 1 \mathrm{H}, \mathrm{OH}-4$ '); 4.84 (dd, $\left.J=10.0,8.0 \mathrm{~Hz}, 1 \mathrm{H}, \mathrm{H}-2^{\prime}\right) ; 4.81$ (d, $J=10.0 \mathrm{~Hz}, 1 \mathrm{H}$, H-1); 4.81 (br s, 1H, OH-3"); 4.78 (d, $J=9.0 \mathrm{~Hz}, \mathrm{H}-1$ '); 4.73 (d, $J=6.5 \mathrm{~Hz}, 1 \mathrm{H}, \mathrm{OH}-2$ "); 4.55 (d, $J$ $=3.5 \mathrm{~Hz}, 1 \mathrm{H}, \mathrm{H}-1$ "); 4.32 (br d, $J=11.5 \mathrm{~Hz}, 1 \mathrm{H}, \mathrm{H}-6 \mathrm{a}) ; 4.22$ (t, $J=6.5 \mathrm{~Hz}, 1 \mathrm{H}, \mathrm{H}-5$ '); 4.07 (dd, $J$ $=11.5,7.0 \mathrm{~Hz}, 1 \mathrm{H}, \mathrm{H}-6 \mathrm{~b}) ; 4.02-3.98$ (m, 3H, H-5, H-6a', H-6b'); 3.81 (t, $J=9.5 \mathrm{~Hz}, 1 \mathrm{H}, \mathrm{H}-4) ; 3.47$ (dd, $J=12.0,7.0 \mathrm{~Hz}, 1 \mathrm{H}, \mathrm{H}-6 \mathrm{a} ") ; 3.88-3.35$ (m, 2H, H-3", H-5”); 3.30 (s, 3H, OCH $)$; 3.19 (m, 1H, H-2"); 3.03 (m, 1H, H-6b"); 2.91 (td, $J=9.5,5.5$ Hz, 1H, H-4”); 2.10, 2.08, 2.02, 2.01, 1.98, 1.94, $1.90\left(7 \times \mathrm{s}, 21 \mathrm{H}, \mathrm{OCOCH}_{3}\right)$, assignments were confirmed by ${ }^{1} \mathrm{H}-{ }^{1} \mathrm{H}$ gCOSY.${ }^{13} \mathrm{C}$ NMR $(125 \mathrm{MHz}$, 
DMSO- $\left.d_{6}\right): \delta=170.2,169.9,169.8,169.4,169.3,169.1,168.7\left(\mathrm{OCOCH}_{3}\right) ; 99.9(\mathrm{C}-1$ '); $99.6(\mathrm{C}-$ 1"); 86.5 (C-1); 76.0 (C-4); 75.5 (C-5); 73.1, 73.0 (C-3, C-3”); 71.9 (C-2"); 71.8 (C-4"); 71.2 (C5’); 70.3 (C-3’); 69.7 (C-5’); 68.9 (C-2'); 67.9 (C-2); 67.1 (C-4'); 62.4 (C-6); 60.8 (C-6'); 54.5 $\left(\mathrm{OCH}_{3}\right) ; 45.3(\mathrm{C}-6 ") ; 20.6,20.5,20.4(2 \mathrm{C}), 20.3(2 \mathrm{C}), 20.2\left(\mathrm{OCOCH}_{3}\right)$, assignments were confirmed by ${ }^{1} \mathrm{H}_{-}{ }^{13} \mathrm{C}$ HSQC. LRMS $\left(\mathrm{ESI}^{+}\right): m / z=898[\mathrm{M}+\mathrm{Na}]^{+}$. HRMS: Calcd for $\mathrm{C}_{33} \mathrm{H}_{49} \mathrm{NO}_{24} \mathrm{SNa}$ 898.2257, Found 898.2292.

\section{Methyl 6-deoxy-6- $N$-(2,2',3,3',4',6,6'-hepta- $O$-acetyl-1- $S$ - $\beta$-D-maltosyl)-6-sulfonylamino- $\alpha$-D-} glucopyranoside (21b). Compound 21b was obtained from compound 21a according to the general procedure 2. Purification by flash chromatography (3:1 acetone/hexane) afforded $\mathbf{2 1 b}$ (87\% yield) as a white solid. $\mathrm{R}_{f}=0.30\left(3: 1\right.$ acetone/hexane). m.p. $=121^{\circ} \mathrm{C} \cdot[\alpha]^{25} \mathrm{D}=+71(c=1.0$, chloroform $)$. ${ }^{1} \mathrm{H}$ NMR (500 MHz, DMSO- $\left.d_{6}\right): \delta=7.47$ (br s, $\left.1 \mathrm{H}, \mathrm{NH}\right) ; 5.44$ (t, $J=9.0 \mathrm{~Hz}, 1 \mathrm{H}, \mathrm{H}-3$ ); 5.29 (d, $J=$ $\left.3.5 \mathrm{~Hz}, 1 \mathrm{H}, \mathrm{H}-1^{\prime}\right) ; 5.22$ (t, $\left.J=10.0 \mathrm{~Hz}, 1 \mathrm{H}, \mathrm{H}-3^{\prime}\right) ; 5.12(\mathrm{t}, J=9.5 \mathrm{~Hz}, 1 \mathrm{H}, \mathrm{H}-2) ; 4.97$ (t, $J=10.0$ Hz, 1H, H-4'); 4.95 (d, $J=6.0 \mathrm{~Hz}, 1 \mathrm{H}, \mathrm{OH}-4$ '); 4.88 (dd, $J=10.5,4.0 \mathrm{~Hz}, 1 \mathrm{H}, \mathrm{H}-2$ '); 4.85 (d, $J=$ $9.5 \mathrm{~Hz}, 1 \mathrm{H}, \mathrm{H}-1$ ); 4.79 (d, $J=5.0 \mathrm{~Hz}, 1 \mathrm{H}, \mathrm{OH}-3$ ”); 4.72 (d, $J=6.5 \mathrm{~Hz}, 1 \mathrm{H}, \mathrm{OH}-2$ ”); 4.55 (d, $J=3.5$ Hz, 1H, H-1"); 4.41 (dd, J = 12.0, 2.0 Hz, 1H, H-6a); 4.18-4.14 (m, 2H, H-6a', H-6b); 4.11 (m, 1H, H-5); 4.01 (dd, $J=12.5,2.5$ Hz, 1H, H-6b'); 3.98 (m, 1H, H-5'); 3.93 (t, $J=9.0$ Hz, 1H, H-4); 3.46 (dd, $J=13.0,4.0 \mathrm{~Hz}, 1 \mathrm{H}, \mathrm{H}-6 \mathrm{a}$ "); 3.38-3.33 (m, 2H, H-3", H-5"); 3.30 (s, 3H, OCH ) $^{2} 3.19$ (ddd, $J$ $=10.0,7.0,4.0 \mathrm{~Hz}, 1 \mathrm{H}, \mathrm{H}-2 ") ; 3.03$ (m, 1H, H6b"); 2.92 (dt, $J=9.0,6.0 \mathrm{~Hz}, 1 \mathrm{H}, \mathrm{H}-4 ") ; 2.07,2.02$, $1.99,1.98,1.95,1.94\left(6 \times \mathrm{s}, 21 \mathrm{H}, \mathrm{OCOCH}_{3}\right)$, assignments were confirmed by ${ }^{1} \mathrm{H}^{-1} \mathrm{H}$ gCOSY.$~{ }^{13} \mathrm{C}$ NMR (125 MHz, DMSO- $\left.d_{6}\right): \delta=170.1,170.0,169.8,169.6,169.5,169.1,168.8\left(\mathrm{OCOCH}_{3}\right) ; 99.6$ (C-1"); 95.7 (C-1'); 86.5 (C-1); 75.0 (C-5); 74.7 (C-3); 73.8 (C-4); 73.1 (C-3”); 71.9 (C-2”); 71.8 (C-4”); 71.2 (C-5”); 69.4 (C-2’); 68.9 (C-3’); 68.3 (C-2); 68.1 (C-5'); 67.8 (C-4'); 63.0 (C-6); 61.4 
(C-6'); $54.5\left(\mathrm{OCH}_{3}\right) ; 45.2$ (C-6"); 20.6, 20.5, 20.4 (2C), 20.3 (2C), $20.2\left(\mathrm{OCOCH}_{3}\right)$, assignments were confirmed by ${ }^{1} \mathrm{H}_{-}{ }^{13} \mathrm{C}$ HSQC. LRMS $\left(\mathrm{ESI}^{+}\right): m / z=898[\mathrm{M}+\mathrm{Na}]^{+}$; LRMS $\left(\mathrm{ESI}^{-}\right): m / z=874$ [M - H] . HRMS: Calcd for $\mathrm{C}_{33} \mathrm{H}_{49} \mathrm{NO}_{24} \mathrm{SNa}$ 898.2257, Found 898.2270.

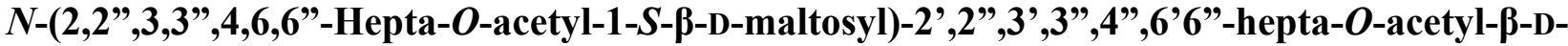

maltosylsulfonylamine (22b). The title compound 22b was prepared from sulfenamide 22a according to the general procedure 2. Purification by flash chromatography (3:2 EtOAc/petroluem spirit) afforded 22b (53\% yield) as a white solid. $\mathrm{R}_{f}=0.30$ (3:2 EtOAc/hexane). m.p. $=117-119^{\circ} \mathrm{C}$. $[\alpha]^{25}{ }_{\mathrm{D}}=+77\left(c=1.0\right.$, chloroform). ${ }^{1} \mathrm{H}$ NMR $\left(500 \mathrm{MHz}, \mathrm{DMSO}-d_{6}\right): \delta=9.04(\mathrm{br} \mathrm{s}, 1 \mathrm{H}, \mathrm{N} H) ; 5.43$ (t, $J=9.0 \mathrm{~Hz}, 1 \mathrm{H}, \mathrm{H}-3) ; 5.33$ (t, $J=9.5 \mathrm{~Hz}, 1 \mathrm{H}, \mathrm{H}-2) ; 5.32$ (d, $J=3.5 \mathrm{~Hz}, 1 \mathrm{H}, \mathrm{H}-1$ ”); 5.26 (m, 1H, H-3'); 5.25 (br s, 1H, H-1"); 5.23 (br t, $J=10.5 \mathrm{~Hz}, 2 \mathrm{H}, 2 \times \mathrm{H} 3$ ”); 4.99, $4.98(2 \times \mathrm{t}, J=9.5 \mathrm{~Hz}, 2 \mathrm{H}$, $2 \times \mathrm{H} 4$ ”); 4.88 (d, $J=10.5 \mathrm{~Hz}, 1 \mathrm{H}, \mathrm{H}-1) ; 4.87,4.86(2 \times \mathrm{t}, J=10.0 \mathrm{~Hz}, 2 \mathrm{H}, 2 \times \mathrm{H} 2$ ”); $4.75(\mathrm{t}, J=$ $9.5 \mathrm{~Hz}, 1 \mathrm{H}, \mathrm{H}-2^{\prime}$ ); 4.66 (d, $\left.J=9.0 \mathrm{~Hz}, 1 \mathrm{H}, \mathrm{H}-1^{\prime}\right) ; 4.56$ (br d, $\left.J=11.5 \mathrm{~Hz}, 1 \mathrm{H}, \mathrm{H}-6 \mathrm{a}\right) ; 4.40$ (br d, $J=$ $12.0 \mathrm{~Hz}, 1 \mathrm{H}, \mathrm{H}-6 \mathrm{a}$ '); 4.19 (dd, $J=13.5,2.5 \mathrm{~Hz}, 1 \mathrm{H}, \mathrm{H}-6 \mathrm{~b}$ ); 4.16 (dd, $J=11.5,2.5 \mathrm{~Hz}, 1 \mathrm{H}, \mathrm{H}-6 \mathrm{~b}$ '); 4.15-4.12 (m, 3H), 4.03-3.93 (m, 5H) (H-5, H-5', 2 × H5", 4 × H6”); 3.89 (t, $J=9.0 \mathrm{~Hz}, 1 \mathrm{H}), 3.85$ $(\mathrm{t}, J=9.5 \mathrm{~Hz}, 1 \mathrm{H})\left(\mathrm{H}-4, \mathrm{H}-4^{\prime}\right) ; 2.09,2.08,2.02,2.01,1.99,1.98,1.96,1.95,1.94,1.93(11 \times \mathrm{s}$, $\left.42 \mathrm{H}, \mathrm{OCOCH}_{3}\right)$, assignments were confirmed by ${ }^{1} \mathrm{H}-{ }^{1} \mathrm{H}$ gCOSY. ${ }^{13} \mathrm{C}$ NMR $\left(125 \mathrm{MHz}\right.$, DMSO- $\left.d_{6}\right)$ : $\delta=170.2,170.0,169.9(2 \mathrm{C}), 169.8(2 \mathrm{C}), 169.7,169.5(2 \mathrm{C}), 169.4,169.2,169.1,169.1,168.9$ $\left(\mathrm{OCOCH}_{3}\right) ; 95.7,95.2\left(\mathrm{C1}{ }^{\prime \prime}\right) ; 86.2(\mathrm{C}-1) ; 81.0\left(\mathrm{C}-1^{\prime}\right) ; 75.1(\mathrm{C}-3$ ') $) 74.8$ (C-3); 74.3, 74.2 (C-5, C5’), 72.7 (2C) (C-5”); 70.8 (C-2'); 69.4 (2C) (C-2”); 68.9 (2C) (C-3”); 68.4 (C-4); 67.8 (C-4'); 67.8 (2C) (C-4"); 67.6 (C-2); 62.8 (C-6’); 62.2 (C-6); 61.5, 61.2 (C-6”); 20.6 (2C), 20.5, 20.4 (4C), 20.3 (5C), $20.2(2 \mathrm{C})\left(\mathrm{OCOCH}_{3}\right)$, assignments were confirmed by ${ }^{1} \mathrm{H}^{13}{ }^{13} \mathrm{C}$ HSQC. LRMS $\left(\mathrm{ESI}^{+}\right): \mathrm{m} / z=$ $1340[\mathrm{M}+\mathrm{Na}]^{+}$. HRMS: Calcd for $\mathrm{C}_{52} \mathrm{H}_{71} \mathrm{NO}_{36} \mathrm{SNa}$ 1340.3369, Found 1340.3367. 


\section{Methyl 6-deoxy-6- $N$-([(2,3,4-tri- $O$-acetyl- $\alpha$-D-xylopyranosyl)-(1 $\rightarrow 6)]-(2,3,4-$-tri- $O$-acetyl- $\beta$-D-}

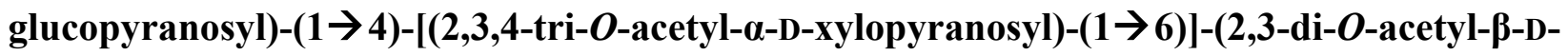

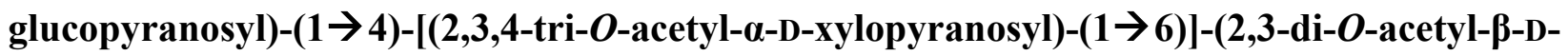
glucopyranosyl)-( $1 \rightarrow 4)-1,2,3,6$-tetra- $O$-acetyl-1-S- $\beta$-D-glucopyranosyl)-6-sulfonylamino- $\alpha$-Dglucopyranoside (23b). The title compound 23b was prepared from sulfenamide $\mathbf{2 3 a}$ according to the general procedure 2. Purification by flash chromatography (5:2 acetone/hexane) afforded 23b (59\% yield) as a white solid. $\mathrm{R}_{f}=0.50\left(5: 2\right.$ acetone/toluene). m.p. $=133-135{ }^{\circ} \mathrm{C} .{ }^{1} \mathrm{H}$ NMR $(500$ MHz, DMSO- $\left.d_{6}\right): \delta=7.46(\mathrm{t}, J=5.5 \mathrm{~Hz}, 1 \mathrm{H}, \mathrm{N} H) ; 5.36(\mathrm{t}, J=9.5 \mathrm{~Hz}, 1 \mathrm{H}) ; 5.30(\mathrm{t}, J=9.5 \mathrm{~Hz}$, $1 \mathrm{H}) ; 5.27(\mathrm{t}, J=9.5 \mathrm{~Hz}, 1 \mathrm{H}) ; 5.25(\mathrm{t}, J=9.5 \mathrm{~Hz}, 1 \mathrm{H}) ; 5.17(\mathrm{t}, J=10.0 \mathrm{~Hz}, 1 \mathrm{H}) ; 5.11(\mathrm{t}, J=9.0 \mathrm{~Hz}$, $1 \mathrm{H}) ; 5.10(\mathrm{t}, J=9.0 \mathrm{~Hz}, 1 \mathrm{H}) ; 5.08-5.05(\mathrm{~m}, 2 \mathrm{H}) ; 5.00-4.72(\mathrm{~m}, 16 \mathrm{H}) ; 4.64(\mathrm{t}, J=9.0 \mathrm{~Hz}, 1 \mathrm{H}) ; 4.55-$ $4.52(\mathrm{~m}, 2 \mathrm{H}) ; 4.49(\mathrm{t}, J=8.5 \mathrm{~Hz}, 1 \mathrm{H}) ; 4.30(\mathrm{~d}, J=11.5 \mathrm{~Hz}, 1 \mathrm{H}) ; 4.08(\mathrm{~m}, 1 \mathrm{H}) ; 3.96(\mathrm{~d}, J=9.0 \mathrm{~Hz}$, $1 \mathrm{H})$; 3.93-3.87 (m, 2H); 3.85-3.61 (m, 17H); 3.51-3.43 (m, 2H); 3.38-3.34 (m, $1 \mathrm{H}) ; 3.29(\mathrm{~s}, 3 \mathrm{H}$, $\left.\mathrm{OCH}_{3}\right) ; 3.17(\mathrm{~m}, 1 \mathrm{H}) ; 3.01$ (ddd, $\left.J=13.0,9.0,3.5 \mathrm{~Hz}, 1 \mathrm{H}\right) ; 2.89$ (br t, $\left.J=9.0 \mathrm{~Hz}, 1 \mathrm{H}\right) ; 2.06,2.05$, 2.04, 2.01, 2.00, 1.99, 1.97, 1.96, 1.95, $1.92\left(12 \times \mathrm{s}, 57 \mathrm{H}, \mathrm{OCOCH}_{3}\right) . \mathrm{LRMS}\left(\mathrm{ESI}^{+}\right): \mathrm{m} / z=2124[\mathrm{M}$ $+\mathrm{Na}]^{+}$.

2-Deoxy-2- $N$-(1-S- $\beta$-D-lactosyl)-2-sulfonylamino- $\alpha, \beta$-D-glucopyranose (19c). The title compound $19 \mathbf{c}$ was obtained from compound $19 \mathrm{~b}$ according to the general procedure 3 as a mixture of $\alpha$ - and $\beta$-anomers $\left(94 \%\right.$ yield; $\alpha / \beta$ 80:20) as a white solid. $\mathrm{R}_{f}=0.16\left(8: 2 \mathrm{CH}_{3} \mathrm{CN} / \mathrm{H}_{2} \mathrm{O}\right)$. m.p. $=171{ }^{\circ} \mathrm{C}$ (decomposition). ${ }^{1} \mathrm{H}$ NMR (500 MHz, DMSO- $d_{6}, \alpha$-anomer): $\delta=6.58$ (d, $J=4.0 \mathrm{~Hz}, 1 \mathrm{H}, \mathrm{OH}-1$ "); $6.55($ br s, $1 \mathrm{H}, \mathrm{NH}) ; 5.39(\mathrm{~d}, J=5.0 \mathrm{~Hz}, 1 \mathrm{H}), 5.07(\mathrm{br} \mathrm{s}, 1 \mathrm{H}), 4.99(\mathrm{br} \mathrm{s}, 1 \mathrm{H})(3 \times \mathrm{OH}) ; 4.99(\mathrm{~d}, J=$ $3.5 \mathrm{~Hz}, 1 \mathrm{H}, \mathrm{H}-1$ ”); $4.93(\mathrm{~m}, 1 \mathrm{H}), 4.90(\mathrm{~d}, J=5.5 \mathrm{~Hz}, 1 \mathrm{H}), 4.83(\mathrm{br} \mathrm{s}, 1 \mathrm{H}), 4.76(\mathrm{~m}, 1 \mathrm{H}), 4.67(\mathrm{t}, J=$ 
$4.0 \mathrm{~Hz}, 1 \mathrm{H}), 4.50(\mathrm{~d}, J=3.5 \mathrm{~Hz}, 1 \mathrm{H}), 4.41(\mathrm{~d}, J=5.5 \mathrm{~Hz}, 1 \mathrm{H})(7 \times \mathrm{OH}) ; 4.38(\mathrm{~d}, J=9.0 \mathrm{~Hz}, 1 \mathrm{H}, \mathrm{H}-$ 1); $4.20\left(\mathrm{~d}, J=7.0 \mathrm{~Hz}, 1 \mathrm{H}, \mathrm{H}-1^{\prime}\right) ; 3.85(\mathrm{dd}, J=11.5,5.0 \mathrm{~Hz}, 1 \mathrm{H}, \mathrm{H}-6 \mathrm{a}) ; 3.62-3.50$ (m, 7H), 3.473.43 (m, 5H), 3.33-3.29 (m, 3H), 3.10 (m, 1H) (H-2, H-2”, H-3, H-3', H-3”, H-4, H-4', H-4”, H-5, H-5', H-5", H-6b, H-6a', H-6b', H-6a", H-6b"); 3.12 (m, 1H, H-2"), assignments were confirmed by ${ }^{1} \mathrm{H}-{ }^{1} \mathrm{H}$ gCOSY. ${ }^{13} \mathrm{C}$ NMR (125 MHz, DMSO- $\left.d_{6}\right): \delta=103.7$ (C-1'); 91.9 (C-1"); $90.0(\mathrm{C}-1)$; 79.8, 79.4, 75.6, 75.5, 73.3, 72.0, 70.9 (2C), 70.7, 70.6, 68.2 (C-2, C-2", C-3, C-3', C-3", C-4, C-4', C-4", C-5, C-5', C-5"); 61.1, 60.5 (2C) (C-6, C-6', C-6"); 58.7 (C-2'), assignments were confirmed by ${ }^{1} \mathrm{H}^{13}{ }^{13} \mathrm{HSQC}$. LRMS $\left(\mathrm{ESI}^{+}\right): m / z=590[\mathrm{M}+\mathrm{Na}]^{+}$; LRMS (ESI) $: m / z=566[\mathrm{M}-\mathrm{H}]-$ HRMS: Calcd for $\mathrm{C}_{18} \mathrm{H}_{33} \mathrm{NO}_{17} \mathrm{SNa} 590.1361$, Found 590.1340.

Methyl 6-deoxy-6- $N$-(1-S- $\beta$-D-lactosyl)-6-sulfonylamino- $\alpha$-D-glucopyranoside (20c). The title compound 20c was obtained from compound 20b as a light orange gum according to the general procedure 3 (98\% yield). $\mathrm{R}_{f}=0.18\left(8: 2 \mathrm{CH}_{3} \mathrm{CN} / \mathrm{H}_{2} \mathrm{O}\right) \cdot[\alpha]^{25}=+62\left(c=1.0\right.$, methanol). ${ }^{1} \mathrm{H}$ NMR (500 MHz, DMSO- $d_{6}, \alpha$-anomer): $\delta=6.79(\mathrm{dd}, J=6.5,5.5 \mathrm{~Hz}, 1 \mathrm{H}, \mathrm{N} H) ; 5.31(\mathrm{~d}, J=6.0 \mathrm{~Hz}, 1 \mathrm{H}$, $\mathrm{O} H-2) ; 5.07$ (d, $\left.J=4.0 \mathrm{~Hz}, 1 \mathrm{H}, \mathrm{OH}-2^{\prime}\right) ; 4.98$ (d, $J=5.5 \mathrm{~Hz}, 1 \mathrm{H}, \mathrm{OH}-4$ ”); 4.78 (m, 2H), 4.75 (d, $J=$ $4.0 \mathrm{~Hz}, 1 \mathrm{H})\left(\mathrm{OH}-3, \mathrm{OH}-3^{\prime}, \mathrm{OH}-3\right.$ ”); 4.72 (d, $J=6.5 \mathrm{~Hz}, 1 \mathrm{H}, \mathrm{OH}-2$ "); 4.64 (t, $J=5.0 \mathrm{~Hz}, 1 \mathrm{H}, \mathrm{OH}-$ 6'); 4.53 (t, $J=6.0 \mathrm{~Hz}, 1 \mathrm{H}, \mathrm{OH}-6$ ); 4.53 (d, $J=3.5 \mathrm{~Hz}, 1 \mathrm{H}, \mathrm{H}-1$ '); 4.49 (d, $\left.J=5.0 \mathrm{~Hz}, 1 \mathrm{H}, \mathrm{O} H-4^{\prime}\right)$; $4.29(\mathrm{~d}, J=9.0 \mathrm{~Hz}, 1 \mathrm{H}, \mathrm{H}-1) ; 4.21$ (d, $J=7.5 \mathrm{~Hz}, 1 \mathrm{H}, \mathrm{H}-1$ '); 3.77 (ddd, $J=12.5,6.0,2.5 \mathrm{~Hz}, 1 \mathrm{H}$, H-6a); 3.64 (br dd, $J=11.5,5.0 \mathrm{~Hz}, 1 \mathrm{H}, \mathrm{H}-6 \mathrm{~b}) ; 3.62$ (m, 1H, H-4'); 3.53 (m, 1H, H-2); 3.52-3.49 (m, 2H, H-6a', H-6b'); 3.47-3.41 (m, 4H, H-3, H-3', H-5, H-6a”); 3.39-3.31 (m, 5H, H-2', H-3”, H4, H-5', H-5"); 3.30 (s, 3H, OCH $H_{3}$ ); 3.20 (ddd, $J=10.0,6.0,3.5$ Hz, 1H, H-2"); 3.05 (ddd, $J=13.0$, 7.5, $5.5 \mathrm{~Hz}, 1 \mathrm{H}, \mathrm{H}-6 \mathrm{~b}$ "); 2.98 (td, $J=9.0,5.5 \mathrm{~Hz}, 1 \mathrm{H}, \mathrm{H}-2$ "), assignments were confirmed by ${ }^{1} \mathrm{H}-{ }^{1} \mathrm{H}$ 
gCOSY. ${ }^{13} \mathrm{C}$ NMR (125 MHz, DMSO- $\left.d_{6}\right): \delta=103.7$ (C-1"); 99.7 (C-1"); 89.1 (C-1); 79.6, 79.0 (C5, C-5’); 75.6, 75.5 (C-3, C-3’); 73.2, 73.1 (C-3”, C-4); 71.9 (C-2”); 71.5 (C-4”); 71.1 (C-5”); 70.5 (2C) (C-2, C-2'); 68.2 (C-4'); 60.4 (C-6'); 60.2 (C-6); $54.6\left(\mathrm{OCH}_{3}\right) ; 44.7$ (C-6”) assignments were confirmed by ${ }^{1} \mathrm{H}^{13}{ }^{13} \mathrm{CHSQC}$. LRMS $\left(\mathrm{ESI}^{+}\right): m / z=583[\mathrm{M}+\mathrm{H}]^{+}$; LRMS (ESI): $m / z=580[\mathrm{M}-\mathrm{H}]^{-}$, 617 [M + Cl] $]^{-}$HRMS: Calcd for $\mathrm{C}_{19} \mathrm{H}_{35} \mathrm{NO}_{17} \mathrm{SNa}$ 604.1518, Found 604.1515.

Methyl 6-deoxy-6- $N$-(-1-S- $\beta$-D-maltosyl)-6-sulfonylamino- $\alpha$-D-glucopyranoside (21c). The title compound 21c was obtained from compound $\mathbf{2 1 b}$ according to the general procedure 3 as a white solid (98\% yield). $\mathrm{R}_{f}=0.34\left(75: 25 \mathrm{CH}_{3} \mathrm{CN} / \mathrm{H}_{2} \mathrm{O}\right) .[\alpha]^{25}{ }_{\mathrm{D}}=+94\left(c=1.0\right.$, methanol). m.p. $=159^{\circ} \mathrm{C}$. ${ }^{1} \mathrm{H}$ NMR (500 MHz, DMSO- $\left.d_{6}\right): \delta=6.84(\mathrm{t}, J=6.0 \mathrm{~Hz}, 1 \mathrm{H}, \mathrm{NH}) ; 5.64(\mathrm{~d}, J=2.5 \mathrm{~Hz}, 1 \mathrm{H}, \mathrm{OH}-4$ '); $5.40\left(\mathrm{~d}, J=6.0 \mathrm{~Hz}, 1 \mathrm{H}, \mathrm{OH}-2^{\prime}\right) ; 5.24(\mathrm{~d}, J=5.5 \mathrm{~Hz}, 1 \mathrm{H}, \mathrm{OH}-2) ; 5.03\left(\mathrm{~d}, J=3.5 \mathrm{~Hz}, 1 \mathrm{H}, \mathrm{H}-1^{\prime}\right)$; 4.99 (d, $J=6.0 \mathrm{~Hz}, 1 \mathrm{H}, \mathrm{OH}-4$ ”); 4.90 (d, $J=5.5 \mathrm{~Hz}, 1 \mathrm{H}, \mathrm{OH}-3) ; 4.87$ (d, $J=4.5 \mathrm{~Hz}, 1 \mathrm{H}) ; 4.79$ (d, $J$ $=4.5 \mathrm{~Hz}, 1 \mathrm{H})\left(\mathrm{O} H-3^{\prime}, \mathrm{OH}-3^{\prime \prime}\right) ; 4.74\left(\mathrm{~d}, J=6.5 \mathrm{~Hz}, 1 \mathrm{H}, \mathrm{OH}-2^{\prime \prime}\right) ; 4.54$ (t, $\left.J=5.5 \mathrm{~Hz}, 1 \mathrm{H}, \mathrm{O} H-6^{\prime}\right)$; $4.53(\mathrm{~d}, J=3.5 \mathrm{~Hz}, 1 \mathrm{H}, \mathrm{H}-1$ ”); 4.49 (t, $J=6.0 \mathrm{~Hz}, 1 \mathrm{H}, \mathrm{OH}-6) ; 4.27$ (d, $J=9.0 \mathrm{~Hz}, 1 \mathrm{H}, \mathrm{H}-1) ; 3.73$ (dd, $J=12.0,6.0 \mathrm{~Hz}, 1 \mathrm{H}, \mathrm{H}-6 \mathrm{a}) ; 3.62(\mathrm{dd}, J=5.5 \mathrm{~Hz}, 1 \mathrm{H}, \mathrm{OH}-3) ; 3.62(\mathrm{dd}, J=10.5,5.0 \mathrm{~Hz}, 1 \mathrm{H}$, H-6a'); 3.59 (m, 1H, H-6b); 3.52 (td, $J=7.0,2.0 \mathrm{~Hz}, 1 \mathrm{H}, \mathrm{H}-4$ '); 3.50 (td, $J=10.0,5.5 \mathrm{~Hz}, 1 \mathrm{H}, \mathrm{H}-$ 2); 3.47-3.42 (m, 3H, H-4, H-6a", H-6b'); 3.39-3.34 (m, 5H, H-3', H-3”, H-5, H-5', H-5”); 3.30 (s, 3H, $\mathrm{OCH}_{3}$ ); 3.23 (m, 1H, H-2'), 3.19 (m, 1H, H-2"); 3.06 (m, 1H, H-6b"); 3.03 (m, 1H, H-3); 2.98 (td, $J=9.0,5.5 \mathrm{~Hz}, 1 \mathrm{H}, \mathrm{H}-4$ "), assignments were confirmed by ${ }^{1} \mathrm{H}-{ }^{1} \mathrm{H}$ gCOSY. ${ }^{13} \mathrm{C}$ NMR $(125$ MHz, DMSO- $\left.d_{6}\right): \delta=100.8$ (C-1'); 99.7 (C-1'); 89.4 (C-1); 79.3, 78.8 (C-5, C-5'); 76.9 (C-4'); 73.6 (C-4); 73.3, 73.1 (C-3’, C-3”); 72.5 (C-2'); 71.9 (C-2”); 71.6 (C-4”); 71.2 (C-5”); 70.3 (C-2); 70.0 (C-3); 60.9 (C-6'); 60.4 (C-6); $54.7\left(\mathrm{OCH}_{3}\right) ; 44.7$ (C-6"), assignments were confirmed by ${ }^{1} \mathrm{H}-$ 
${ }^{13} \mathrm{C}$ HSQC. LRMS $\left(\mathrm{ESI}^{+}\right): m / z=599\left[\mathrm{M}+\mathrm{NH}_{4}\right]^{+}, 604[\mathrm{M}+\mathrm{Na}]^{+}$. HRMS: Calcd for $\mathrm{C}_{19} \mathrm{H}_{35} \mathrm{NO}_{17} \mathrm{SNa} 604.1518$, Found 604.1495 .

$N$-(1-S- $\beta$-D-Maltosyl)- $\beta$-D-maltosylsulfonylamine (22c). The title compound 22c was obtained from compound $\mathbf{2 2 b}$ according to the general procedure 3 as a highly hygroscopic white solid ( $99 \%$ yield). $\mathrm{R}_{f}=0.35\left(7: 3 \mathrm{CH}_{3} \mathrm{CN} / \mathrm{H}_{2} \mathrm{O}\right) \cdot[\alpha]_{\mathrm{D}}^{25}=+131\left(c=1.0\right.$, methanol). ${ }^{1} \mathrm{H}$ NMR $(500 \mathrm{MHz}$, DMSO- $\left.d_{6}\right): \delta=5.74$ (br s, 1H), 5.67 (br s, 1H), 5.58 (br s, $\left.1 \mathrm{H}\right), 5.45$ (br s, 2H), 5.38 (br s, $\left.1 \mathrm{H}\right)(6 \times$ $\mathrm{OH}) ; 5.02(\mathrm{~m}, 3 \mathrm{H}, 2 \times \mathrm{H}-1$ ”, $\mathrm{NH}) ; 4.91($ br s, $3 \mathrm{H}, 3 \times \mathrm{OH}) ; 4.68(\mathrm{br} \mathrm{s}), 4.52(\mathrm{br} \mathrm{s}, 4 \mathrm{H})(5 \times \mathrm{OH})$; $4.27(\mathrm{~d}, J=7.5 \mathrm{~Hz}, 1 \mathrm{H}), 4.26(\mathrm{~d}, J=9.0 \mathrm{~Hz}, 1 \mathrm{H})\left(\mathrm{H}-1, \mathrm{H}-1^{\prime}\right) ; 3.82(\mathrm{t}, J=8.0 \mathrm{~Hz}, 1 \mathrm{H}), 3.76-3.68$ (m, 3H), 3.62-3.54 (m, 5H), $3.51(\mathrm{~d}, J=7.5 \mathrm{~Hz}, 1 \mathrm{H}), 3.48-3.42(\mathrm{~m}, 6 \mathrm{H}), 3.29(\mathrm{~m}, 1 \mathrm{H}), 3.23-3.21$ (m, 4H), $3.13(\mathrm{t}, J=7.5 \mathrm{~Hz}, 1 \mathrm{H}), 3.06(\mathrm{br} \mathrm{t}, J=7.5 \mathrm{~Hz}, 2 \mathrm{H})$, assignments were confirmed by ${ }^{1} \mathrm{H}-{ }^{1} \mathrm{H}$ gCOSY. ${ }^{13} \mathrm{C}$ NMR (125 MHz, DMSO- $\left.d_{6}\right): \delta=100.8$ (2C) $(2 \times \mathrm{C}-1$ "); 100.1 (C-1'); $90.3(\mathrm{C}-1)$; 79.3, 79.2, 78.9, 77.1, 77.0, 76.6, 73.6, 73.5, 73.3 (2C), 72.5 (3C), 70.2, 69.9 (2C); $60.8(3 \mathrm{C}), 60.5$ (C-6, C-6', $2 \times$ C-6"), assignments were confirmed by ${ }^{1} \mathrm{H}^{13}{ }^{13} \mathrm{C}$ HSQC. LRMS $\left(\mathrm{ESI}^{+}\right): \mathrm{m} / z=752[\mathrm{M}$ $+\mathrm{Na}]^{+}$. HRMS: Calcd for $\mathrm{C}_{24} \mathrm{H}_{43} \mathrm{NO}_{22} \mathrm{SNa}$ 752.1890, Found 752.1884.

\section{Methyl 3-deoxy-3- $N$-(2,3,4,6-tetra- $O$-acetyl-1-S- $\beta$-D-glucopyranosyl)- $\alpha, \beta$-D-allopyranoside}

(24a). Compound 24a was obtained from compound 1 and amine 12 according to the general procedure 1. Purification by flash chromatography (1:1 acetone/petroleum spirit) afforded 24a (39\% yield) as a light yellow gum. Compound 24a was used as reagent for general procedure 2 without further purification. $\mathrm{R}_{f}=0.45\left(2: 1\right.$ acetone/hexane). LRMS $\left(\mathrm{ESI}^{+}\right): m / z=578[\mathrm{M}+\mathrm{Na}]^{+}$. HRMS: Calcd for $\mathrm{C}_{21} \mathrm{H}_{33} \mathrm{NO}_{14} \mathrm{SNa} 578.1514$, Found 578.1534 
Methyl 3-deoxy-3- $N$-(2,3,4,6-tetra- $O$-acetyl-1-S- $\beta$-D-glucopyranosyl)-3-sulfonylamino- $\alpha, \beta$-Dallopyranoside (24b). Compound $\mathbf{2 4 b}$ was obtained from compound $\mathbf{2 4 a}$ according to the general procedure 2. Purification by flash chromatography (1:1 acetone/petroleum spirit) afforded $24 \mathbf{a}$ as a mixture of $\alpha$ - and $\beta$-anomers $(68 \%$ yield; $\alpha / \beta 20: 80)$ as a colorless gum. $\mathrm{R}_{f}=0.26(1: 1$ acetone/hexane). ${ }^{1} \mathrm{H}$ NMR (500 MHz, DMSO- $d_{6}, \beta$-anomer): $\delta=7.21$ (br s, $\left.1 \mathrm{H}, \mathrm{N} H\right) ; 5.33(\mathrm{t}, J=$ $9.5 \mathrm{~Hz}, 1 \mathrm{H}, \mathrm{H}-3) ; 5.26$ (t, $J=9.5 \mathrm{~Hz}, 1 \mathrm{H}, \mathrm{H}-5) ; 5.19$ (d, $J=6.0 \mathrm{~Hz}, 1 \mathrm{H}, \mathrm{OH}-2$ '); 4.96 (t, $J=9.5$ Hz, 1H, H-4); 4.94 (d, $J=10.0 \mathrm{~Hz}, 1 \mathrm{H}, \mathrm{H}-1) ; 4.85$ (d, $J=6.5 \mathrm{~Hz}, 1 \mathrm{H}, \mathrm{OH}-4$ '); 4.57 (t, $J=6.0 \mathrm{~Hz}$, 1H, OH-6'); 4.50 (d, $\left.J=6.0 \mathrm{~Hz}, 1 \mathrm{H}, \mathrm{H}-1^{\prime}\right)$; 4.22 (dd, $\left.J=12.5,5.0 \mathrm{~Hz}, 1 \mathrm{H}, \mathrm{H}-6 \mathrm{a}\right) ; 4.03-3.99$ (m, 2H, H-5, H-6b); 3.72 (br s, 1H, H-3'); 3.67-3.57 (m, 2H, H-5', H-6a'); 3.56 (m, 1H, H-4'); 3.46 (br t, $\left.J=6.0 \mathrm{~Hz}, 1 \mathrm{H}, \mathrm{H}-2^{\prime}\right) ; 3.41\left(\mathrm{~m}, 1 \mathrm{H}, \mathrm{H}-6 \mathrm{~b}^{\prime}\right) ; 3.34\left(\mathrm{~s}, 3 \mathrm{H}, \mathrm{OCH}_{3}\right) ; 2.01,1.98,1.96,1.95(4 \times \mathrm{s}$, $\left.12 \mathrm{H}, \mathrm{OCOCH}_{3}\right)$, assignments were confirmed by ${ }^{1} \mathrm{H}-{ }^{1} \mathrm{H}$ gCOSY.${ }^{13} \mathrm{C} \mathrm{NMR}\left(125 \mathrm{MHz}\right.$, DMSO- $\left.d_{6}\right)$ : $\delta=170.0,169.5,169.1,168.9\left(\mathrm{OCOCH}_{3}\right) ; 101.4\left(\mathrm{C}-1^{\prime}\right) ; 86.0(\mathrm{C}-1) ; 76.7(\mathrm{C}-5$ '); $74.7(\mathrm{C}-5) ; 73.0$

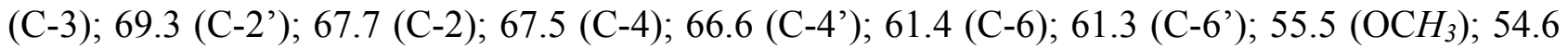
(C-3’); 20.5, 20.3 (2C), $20.2\left(\mathrm{OCOCH}_{3}\right)$, assignments were confirmed by ${ }^{1} \mathrm{H}_{-}{ }^{13} \mathrm{C}$ HSQC. LRMS $\left(\mathrm{ESI}^{+}\right): m / z=610[\mathrm{M}+\mathrm{Na}]^{+}$. HRMS: Calcd for $\mathrm{C}_{21} \mathrm{H}_{33} \mathrm{NO}_{16} \mathrm{SNa}$ 610.1412, Found 610.1386.

\section{Methyl 6-deoxy-6- $N$-(2,3,4,6-tetra- $O$-acetyl-1-S- $\alpha$-D-mannopyranosyl)- $\alpha$-D-glucopyranoside}

(25a). Compound 25a was obtained from compounds 5 and $\mathbf{1 0}$ according to the general procedure 1. Purification by flash chromatography $\left(9: 1 \mathrm{CH}_{2} \mathrm{Cl}_{2} / \mathrm{MeOH}\right)$ afforded $25 \mathbf{a}(76 \%$ yield) as a light yellow gum. Compound 25a was used as reagent for general procedure 2 without further purification. $\mathrm{R}_{f}=0.45\left(9: 1 \mathrm{CH}_{2} \mathrm{Cl}_{2} / \mathrm{MeOH}\right) . \mathrm{LRMS}\left(\mathrm{ESI}^{+}\right): m / z=578[\mathrm{M}+\mathrm{Na}]^{+}$. HRMS: Calcd for $\mathrm{C}_{21} \mathrm{H}_{33} \mathrm{NO}_{14} \mathrm{SNa}$ 578.1514, Found 578.1497. 
Methyl 6-deoxy-6- $N$-(2,3,4,6-tetra- $O$-acetyl-1-S- $\alpha$-D-mannopyranosyl)-6-sulfonylamino- $\alpha$-Dglucopyranoside (25b). Compound $\mathbf{2 5 b}$ was obtained from compound 25a according to the general procedure 2. Purification by flash chromatography $\left(9: 1 \mathrm{CH}_{2} \mathrm{Cl}_{2} / \mathrm{MeOH}\right)$ afforded $\mathbf{2 5 b}(80 \%$ yield $)$ as a light yellow gum. $\mathrm{R}_{f}=0.38\left(9: 1 \mathrm{CH}_{2} \mathrm{Cl}_{2} / \mathrm{MeOH}\right) .{ }^{1} \mathrm{H}$ NMR $\left(500 \mathrm{MHz}, \mathrm{DMSO}-d_{6}\right): \delta=7.89(\mathrm{t}$, $J=6.0 \mathrm{~Hz}, 1 \mathrm{H}, \mathrm{NH}) ; 5.71(\mathrm{dd}, J=4.0,1.5 \mathrm{~Hz}, 1 \mathrm{H}, \mathrm{H}-2) ; 5.45(\mathrm{dd}, J=10.0,4.0 \mathrm{~Hz}, 1 \mathrm{H}, \mathrm{H}-3) ; 5.21$ (t, $J=10.0 \mathrm{~Hz}, 1 \mathrm{H}, \mathrm{H}-4) ; 5.10(\mathrm{~d}, J=1.0 \mathrm{~Hz}, 1 \mathrm{H}, \mathrm{H}-1) ; 4.52(\mathrm{~d}, J=3.5 \mathrm{~Hz}, 1 \mathrm{H}, \mathrm{H}-1$ '); 4.51 (m, 1H, H-5); 4.16 (dd, $J=12.5,4.0 \mathrm{~Hz}, 1 \mathrm{H}, \mathrm{H}-6 \mathrm{a}) ; 4.12$ (dd, $J=12.5,2.5 \mathrm{~Hz}, 1 \mathrm{H}, \mathrm{H}-6 \mathrm{~b}) ; 3.47$ (m, 1H, H6a'); 3.41-3.36 (m, 2H, H-3', H-5'); 3.30 (s, 3H, OCH $)$; 3.20 (dd, J=9.5, 3.5 Hz, 1H, H-2'); 3.04 (m, 1H, H-6b'); 2.95 (t, $J=9.5 \mathrm{~Hz}, 1 \mathrm{H}, \mathrm{H}-4$ '); 2.13, 2.03, 2.02, $1.94\left(4 \times \mathrm{s}, 12 \mathrm{H}, \mathrm{OCOCH}_{3}\right)$, assignments were confirmed by ${ }^{1} \mathrm{H}-{ }^{1} \mathrm{H}$ gCOSY. ${ }^{13} \mathrm{C}$ NMR (125 MHz, DMSO- $\left.d_{6}\right): \delta=169.9,169.4$, 169.3, 169.2 ( $\left.\mathrm{OCOCH}_{3}\right)$; $99.8\left(\mathrm{C}-1^{\prime}\right) ; 87.1$ (C-1); 73.0 (C-5'); 72.1 (C-5); 71.8 (C-2’); 71.5 (C-4'); 71.2 (C-3’); 68.2 (C-3); 65.9 (C-2); 64.4 (C-4); 61.7 (C-6); $54.6\left(\mathrm{OCH}_{3}\right) ; 44.3$ (C-6’); 20.5, 20.4, $20.3(2 \mathrm{C})\left(\mathrm{OCOCH}_{3}\right)$, assignments were confirmed by ${ }^{1} \mathrm{H}-{ }^{13} \mathrm{C}$ HSQC. LRMS $\left(\mathrm{ESI}^{+}\right): \mathrm{m} / z=610[\mathrm{M}$ $+\mathrm{Na}]^{+}$. HRMS: Calcd for $\mathrm{C}_{21} \mathrm{H}_{33} \mathrm{NO}_{16} \mathrm{SNa}$ 610.1412, Found 610.1401.

\section{3-Deoxy-3- $N$-(2,3,4,6-tetra- $O$-acetyl-1- $S$ - $\beta$-D-glucopyranosyl)-1,2:5,6-di- $O$-isopropylidene- $\alpha$-D-}

allofuranose (26a). Compound 26a was obtained from compounds 1 and 13 according to the general procedure 1. Purification by flash chromatography (1:3 EtOAc/petroleum spirit) afforded the title compound $\mathbf{2 6 a}$ as a light off-white solid (73\% yield). $\mathrm{R}_{f}=0.17$ (1:2 EtOAc/petroleum spirit). m.p. $=109^{\circ} \mathrm{C} .[\alpha]_{\mathrm{D}}^{25}=-5(c=1.0$, chloroform $) .{ }^{1} \mathrm{H}$ NMR $\left(500 \mathrm{MHz}, \mathrm{DMSO}-d_{6}\right): \delta=5.79$ (d, $J=3.5 \mathrm{~Hz}, 1 \mathrm{H}, \mathrm{H}-1$ ”); $5.51(\mathrm{t}, J=9.5 \mathrm{~Hz}, 1 \mathrm{H}, \mathrm{H}-2) ; 5.38(\mathrm{t}, J=9.5 \mathrm{~Hz}, 1 \mathrm{H}, \mathrm{H}-3) ; 4.95(\mathrm{t}, J=$ $10.0 \mathrm{~Hz}, 1 \mathrm{H}, \mathrm{H}-4) ; 4.77$ (t, $J=4.5 \mathrm{~Hz}, 1 \mathrm{H}, \mathrm{H}-2$ '”) 4.46 (d, $J=10.0 \mathrm{~Hz}, 1 \mathrm{H}, \mathrm{H}-1) ; 4.27$ (m, 1H, H5”); 4.25 (m, 1H, H-6a); 4.04-4.01 (m, 2H, H-5, H-6b); 3.94 (t, J= 7.5 Hz, 1H, H-6a”); 3.82 (t, $J=$ 
$7.5 \mathrm{~Hz}, 1 \mathrm{H}, \mathrm{H}-6 \mathrm{~b} ”) ; 3.74$ (dd, $J=10.0,2.0 \mathrm{~Hz}, 1 \mathrm{H}, \mathrm{H}-4 ”) ; 3.59$ (d, J=10.0 Hz, 1H, NH); 3.19 (td, $J=10.0,50.0 \mathrm{~Hz}, 1 \mathrm{H}, \mathrm{H}-3$ ”); 2.04, 2.02, $1.97\left(3 \times \mathrm{s}, 12 \mathrm{H}, \mathrm{OCOCH}_{3}\right) ; 1.48,1.39,1.34,1.27(4 \times \mathrm{s}$, $\left.12 \mathrm{H}, \mathrm{C}\left(\mathrm{CH}_{3}\right)_{2}\right)$, assignments were confirmed by ${ }^{1} \mathrm{H}-{ }^{1} \mathrm{H}$ gCOSY. ${ }^{13} \mathrm{C}$ NMR $\left(125 \mathrm{MHz}\right.$, DMSO- $\left.d_{6}\right): \delta$ $=170.2,169.9,169.5,169.1\left(\mathrm{OCOCH}_{3}\right) ; 111.1,108.6\left(C\left(\mathrm{CH}_{3}\right)_{2}\right) ; 104.0(\mathrm{C}-1$ '”); $86.1(\mathrm{C}-1) ; 78.8(\mathrm{C}-$ 2”); 76.6 (C-4”); 74.9 (C-5”); 74.3 (C-5); 73.0 (C-3); 67.6 (C-4); 67.4 (C-2); 66.8 (C-3”); 63.8 (C-

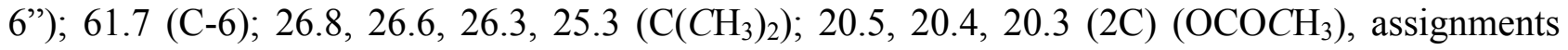
were confirmed by ${ }^{1} \mathrm{H}_{-}{ }^{13} \mathrm{C}$ HSQC. LRMS $\left(\mathrm{ESI}^{+}\right): m / z=644[\mathrm{M}+\mathrm{Na}]^{+}$. HRMS: Calcd for $\mathrm{C}_{26} \mathrm{H}_{39} \mathrm{NO}_{14} \mathrm{SNa} 644.1983$, Found 644.1953.

\section{3-Deoxy-3- $N$-(2,3,4,6-tetra- $O$-acetyl-1-S- $\beta$-D-glucopyranosyl)-1,2:5,6-di- $O$-isopropylidene-3-}

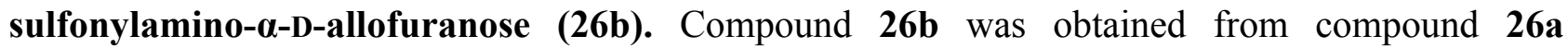
according to the general procedure 2 . Purification by flash chromatography $(1: 2$ EtOAc/petroleum spirit) afforded the title compound $\mathbf{2 6} \mathbf{b}$ as a light off-white solid (83\% yield). $\mathrm{R}_{f}=0.33$ (1:1 EtOAc/petroleum spirit). m.p. $=174{ }^{\circ} \mathrm{C} .[\alpha]^{25} \mathrm{D}=+23(c=1.0$, chloroform $) .{ }^{1} \mathrm{H}$ NMR $(500 \mathrm{MHz}$, DMSO- $\left.d_{6}\right): \delta=7.40(\mathrm{~d}, J=9.5 \mathrm{~Hz}, 1 \mathrm{H}, \mathrm{N} H) ; 5.80\left(\mathrm{~d}, J=3.5 \mathrm{~Hz}, 1 \mathrm{H}, \mathrm{H}-1^{\prime}\right) ; 5.43$ (m, 1H, H-3); $5.41(\mathrm{~m}, 1 \mathrm{H}, \mathrm{H}-2) ; 5.00$ (t, $J=9.5 \mathrm{~Hz}, 1 \mathrm{H}, \mathrm{H}-4) ; 4.96(\mathrm{~d}, J=9.0 \mathrm{~Hz}, 1 \mathrm{H}, \mathrm{H}-1) ; 4.66$ (t, $J=4.0 \mathrm{~Hz}$, 1H, H-2'); 4.33 (dd, $J=12.5,4.0 \mathrm{~Hz}, 1 \mathrm{H}, \mathrm{H}-6 \mathrm{a}) ; 4.26$ (td, $J=7.0,2.5 \mathrm{~Hz}, 1 \mathrm{H}, \mathrm{H}-5$ '); 4.15 (br td, $J$ $=10.0,3.0 \mathrm{~Hz}, 1 \mathrm{H}, \mathrm{H}-5) ; 4.03$ (m, 1H, H-6b); 4.02 (m, 1H, H-4'); 3.92 (t, J = 7.5 Hz, 1H, H-6a'); $3.85\left(\mathrm{t}, J=7.5 \mathrm{~Hz}, 1 \mathrm{H}, \mathrm{H}-6 \mathrm{~b}^{\prime}\right) ; 3.62\left(\mathrm{td}, J=9.5,5.0 \mathrm{~Hz}, 1 \mathrm{H}, \mathrm{H}-3^{\prime}\right) ; 2.02,1.98,1.97,1.96(4 \times \mathrm{s}$, $\left.12 \mathrm{H}, \mathrm{OCOCH}_{3}\right) ; 1.49,1.35,1.33,1.27\left(4 \times \mathrm{s}, 12 \mathrm{H}, \mathrm{C}\left(\mathrm{CH}_{3}\right)_{2}\right)$, assignments were confirmed by ${ }^{1} \mathrm{H}-$ ${ }^{1} \mathrm{H}$ gCOSY. ${ }^{13} \mathrm{C}$ NMR $\left(125 \mathrm{MHz}, \mathrm{DMSO}-d_{6}\right): \delta=169.9,169.5,169.3,169.0\left(\mathrm{OCOCH}_{3}\right) ; 111.5$,

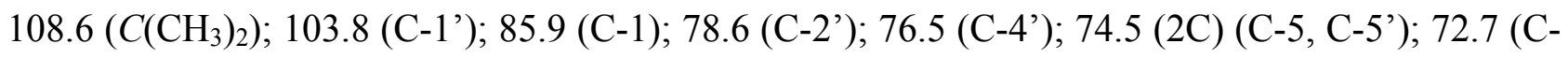
3); 67.6 (C-2); 67.2 (C-4); 63.6 (C-6’); 61.1 (C-6); 55.9 (C-3'); 26.6, 26.5, 26.1, $25.2\left(\mathrm{C}^{\prime}\left(\mathrm{CH}_{3}\right)_{2}\right)$; 
20.5, 20.4, $20.2(2 \mathrm{C})\left(\mathrm{OCOCH}_{3}\right)$, assignments were confirmed by ${ }^{1} \mathrm{H}_{-}{ }^{13} \mathrm{C}$ HSQC. LRMS (ESI $\left.{ }^{+}\right): \mathrm{m} / \mathrm{z}$ $=671\left[\mathrm{M}+\mathrm{NH}_{4}\right]^{+}, 676[\mathrm{M}+\mathrm{Na}]^{+}$. HRMS: Calcd for $\mathrm{C}_{26} \mathrm{H}_{39} \mathrm{NO}_{16} \mathrm{SNa}$ 676.1882, Found 676.1871.

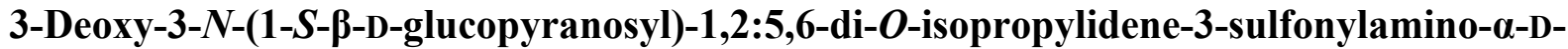

allofuranose (26c). The title compound $26 \mathbf{c}$ was obtained from compound $26 \mathbf{b}$ according to the general procedure 3 . The lyophilized compound was obtained as a colorless gum ( $95 \%$ yield). $\mathrm{R}_{f}=$ $0.79\left(8: 2 \mathrm{CH}_{2} \mathrm{Cl}_{2} / \mathrm{MeOH}\right) .{ }^{1} \mathrm{H}$ NMR (500 MHz, DMSO- $\left.d_{6}\right): \delta=7.27(\mathrm{~s}, 2 \mathrm{H}, \mathrm{N} H) ; 5.70(\mathrm{~d}, J=3.5$ $\left.\mathrm{Hz}, 1 \mathrm{H}, \mathrm{H}-1^{\prime}\right) ; 5.16(\mathrm{~d}, J=3.5 \mathrm{~Hz}, 1 \mathrm{H}, \mathrm{OH}-3) ; 5.04(\mathrm{~d}, J=5.0 \mathrm{~Hz}, 1 \mathrm{H}, \mathrm{OH}-4) ; 4.93$ (br s, $1 \mathrm{H}, \mathrm{OH}-$ 2); 4.67 (t, $J=3.5 \mathrm{~Hz}, 1 \mathrm{H}, \mathrm{H}-2$ '); 4.38 (t, $J=6.0 \mathrm{~Hz}, 1 \mathrm{H}, \mathrm{OH}-6) ; 4.29$ (td, $J=7.0,1.5 \mathrm{~Hz}, 1 \mathrm{H}, \mathrm{H}-$ 5'); 4.23 (d, $J=8.5 \mathrm{~Hz}, 1 \mathrm{H}, \mathrm{H}-1) ; 3.96$ (dd, $J=10.0,1.5 \mathrm{~Hz}, 1 \mathrm{H}, \mathrm{H}-4$ '); 3.91 (t, $J=7.5 \mathrm{~Hz}, 1 \mathrm{H}, \mathrm{H}-$ 6a'); 3.85 (t, $\left.J=7.5 \mathrm{~Hz}, 1 \mathrm{H}, \mathrm{H}-6 \mathrm{~b}^{\prime}\right) ; 3.68$ (dd, $\left.J=10.5,6.5 \mathrm{~Hz}, 1 \mathrm{H}, \mathrm{H}-6 \mathrm{a}\right) ; 3.58$ (m, 1H, H-3'); 3.53-3.49 (m, 2H, H-2, H-6b); 3.26-3.24 (m, 2H, H-3, H-5); 3.15 (m, 1H, H-4); 1.45, 1.34, 1.27, $1.23\left(4 \times \mathrm{s}, 12 \mathrm{H}, \mathrm{C}\left(\mathrm{CH}_{3}\right)_{2}\right)$, assignments were confirmed by ${ }^{1} \mathrm{H}-{ }^{1} \mathrm{H}$ gCOSY. LRMS $\left(\mathrm{ESI}^{+}\right): \mathrm{m} / z=$ $503\left[\mathrm{M}+\mathrm{NH}_{4}\right]^{+}, 508[\mathrm{M}+\mathrm{Na}]^{+}$.

\section{3-Deoxy-3- $N$-(1-S- $\beta$-D-glucopyranosyl)-1,2-O-isopropylidene-3-sulfonylamino- $\alpha$-D-}

allofuranose (26d). A suspension of compound 26c ( $35 \mathrm{mg}, 72.1 \mu \mathrm{mol})$ in water $(2 \mathrm{~mL})$ was stirred gently overnight in presence of Amberlite IR $-120 \mathrm{H}^{+}$resin $(350 \mathrm{mg})$. The reaction mixture was filtered to remove the resin, the filtrate was evaporated to dryness in presence of silica. Purification by solid addition flash chromatography $\left(8: 2 \mathrm{CH}_{2} \mathrm{Cl}_{2} / \mathrm{MeOH}\right)$ afforded the title compound $\mathbf{2 6 d}$ as a colorless gum (20 mg, $44.9 \mu$ mol, $63 \%$ yield). $\mathrm{R}_{f}=0.13\left(8: 2 \mathrm{CH}_{2} \mathrm{Cl}_{2} / \mathrm{MeOH}\right) .{ }^{1} \mathrm{H}$ NMR (500 MHz, DMSO- $\left.d_{6}\right): \delta=7.05(\mathrm{br} \mathrm{s}, 1 \mathrm{H}, \mathrm{N} H) ; 5.65\left(\mathrm{~d}, J=3.5 \mathrm{~Hz}, 1 \mathrm{H}, \mathrm{H}-1^{\prime}\right) ; 5.13(\mathrm{~d}, J=4.5 \mathrm{~Hz}, 1 \mathrm{H}, \mathrm{O} H-3)$; $5.03(\mathrm{~d}, J=5.0 \mathrm{~Hz}, 1 \mathrm{H}, \mathrm{OH}-4) ; 4.87$ (br s, $1 \mathrm{H}, \mathrm{OH}-2) ; 4.83($ br s, $1 \mathrm{H}, \mathrm{OH}-6) ; 4.66(4, J=4.0 \mathrm{~Hz}$, 
1H, H-2'); 4.48 (br s, 1H, OH-5'); 4.45 (br t, J=5.5 Hz, 1H, OH-6'); 4.21 (d, J=9.5 Hz, 1H, H-1); $3.92\left(\mathrm{dd}, J=9.5,4.0 \mathrm{~Hz}, 1 \mathrm{H}, \mathrm{H}-3^{\prime}\right) ; 3.88\left(\mathrm{dd}, J=10.0,2.0 \mathrm{~Hz}, 1 \mathrm{H}, \mathrm{H}-4^{\prime}\right) ; 3.75$ (br t, $J=6.5 \mathrm{~Hz}$, 1H, H-6a); 3.69 (ddd, $J=12.0,6.5,1.5 \mathrm{~Hz}, 1 \mathrm{H}, \mathrm{H}-5$ '); 3.56 (d, $J=9.5 \mathrm{~Hz}, 1 \mathrm{H}, \mathrm{H}-2) ; 3.52$ (dd, $J=$ 13.0, 5.5 Hz, 1H, H-6b); 3.49 (dd, $J=11.0,5.0 \mathrm{~Hz}, 1 \mathrm{H}, \mathrm{H}-6 \mathrm{~b}$ '); 3.42 (dd, $J=10.5,7.5 \mathrm{~Hz}, 1 \mathrm{H}, \mathrm{H}-$ $6 \mathrm{~b}$ ); $3.26(\mathrm{td}, J=9.5,4.0 \mathrm{~Hz}, 1 \mathrm{H}, \mathrm{H}-3) ; 3.24(\mathrm{~m}, 1 \mathrm{H}, \mathrm{H}-5) ; 3.15$ (td, $J=9.5,4.5 \mathrm{~Hz}, 1 \mathrm{H}, \mathrm{H}-4)$; 1.45, $1.26\left(2 \times \mathrm{s}, 6 \mathrm{H}, \mathrm{C}\left(\mathrm{CH}_{3}\right)_{2}\right)$, assignments were confirmed by ${ }^{1} \mathrm{H}^{-}{ }^{1} \mathrm{H}$ gCOSY. LRMS $\left(\mathrm{ESI}^{+}\right): \mathrm{m} / z$ $=468[\mathrm{M}+\mathrm{Na}]^{+}$.

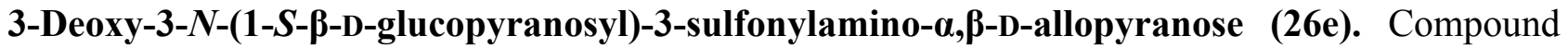
26d (20 mg, $44.9 \mu \mathrm{mol})$ was solubilized in 50\% aqueous trifluoroacetic acid ( $3 \mathrm{~mL})$ and stirred at $\mathrm{rt}$ for $24 \mathrm{~h}$. The mixture was evaporated to dryness in the presence of silica. Purification by flash chromatography $\left(85: 15 \mathrm{CH}_{3} \mathrm{CN} / \mathrm{H}_{2} \mathrm{O}\right)$ afforded the title compound 26e as a mixture of $\alpha$ - and $\beta$ anomers (13 mg, $32.1 \mu \mathrm{mol}, 72 \%$ yield; $\alpha / \beta$ 70:30) as a light yellow gum. $\mathrm{R}_{f}=0.39$ (8:2 $\left.\mathrm{CH}_{3} \mathrm{CN} / \mathrm{H}_{2} \mathrm{O}\right) .{ }^{1} \mathrm{H}$ NMR (500 MHz, DMSO- $d_{6}, \alpha$-anomer): $\delta=6.60(\mathrm{~d}, J=9.5 \mathrm{~Hz}, 1 \mathrm{H}, \mathrm{N} H) ; 4.96$ (br s, 1H, H-1'); 4.28 (d, J=9.5 Hz, 1H, H-1); 4.01 (td, $J=8.0,5.0 \mathrm{~Hz}, 1 \mathrm{H}, \mathrm{H}-3$ '); 3.81 (m, 1H, H2'), 3.78-3.19 (m, 7H, H-4', H-5, H-5', H-6a, H-6a', H-6b, H-6b'); 3.50 (m, H-2); 3.28 (t, J=9.0 $\mathrm{Hz}, 1 \mathrm{H}, \mathrm{H}-3) ; 3.01(\mathrm{t}, J=9.0 \mathrm{~Hz}, 1 \mathrm{H}, \mathrm{H}-4)$, assignments were confirmed by ${ }^{1} \mathrm{H}-{ }^{1} \mathrm{H}$ gCOSY.${ }^{13} \mathrm{C}$ NMR (125 MHz, DMSO- $d_{6}, \alpha$-anomer): $\delta=101.2$ (C-1'); 86.7 (C-1); 81.3, 81.2 (C-5, C-5'); 77.2 (C-3); 74.3 (C-2’); 72.2 (C-4'); 70.3 (C-2); 69.8 (C-4); 62.0, 61.2 (C-6, C-6'); 54.0 (C-3’), assignments were confirmed by ${ }^{1} \mathrm{H}_{-}{ }^{13} \mathrm{C}$ HSQC. LRMS $\left(\mathrm{ESI}^{+}\right): m / z=428[\mathrm{M}+\mathrm{Na}]^{+}$. HRMS: Calcd for $\mathrm{C}_{12} \mathrm{H}_{23} \mathrm{NO}_{12} \mathrm{SNa} 428.0833$, Found 428.0847.

\section{Acknowledgment}


The Australian Research Council is gratefully acknowledged for financial support (Grant numbers DP0877554 and LE0237908 to S.-A.P.).

\section{Supporting Information Available}

${ }^{1} \mathrm{H}$ and ${ }^{13} \mathrm{C}$ NMR spectra of compounds 14a-17a, 19a, 21a-23a, 26a, 14b-26b, 14c-22c, 26c-e. This material is available free of charge via the Internet at http://pubs.acs.org.

\section{References}

(1) Ernst, B.; Magnani, J. Nat. Rev. Drug Discov. 2009, 8, 661.

(2) Boltje, T. J.; Buskas, T.; Boons, G.-J. Nat. Chem. 2009, 1, 611.

(3) Williams, N. R.; Davison, B. E.; Ferrier, R. J.; Furneaux, R. H. Carbohydr. Chem. 1985, 17, 15.

(4) Paterson, D. E.; Griffin, F. K.; Alcaraz, M.-L.; Taylor, R. J. K. Eur. J. Org. Chem. 2002, 7, 1323.

(5) Turnbull, W. B.; Fascione, M. A.; Stalford, S. A. Sci. Synth. 2007, 29, 923.

(6) Morais, G. R.; Humphrey, A. J.; Falconer, R. A.Tetrahedron 2008, 64, 7426.

(7) Tony, K. A.; Denton, R. W.; Dilhas, A.; Jimenez-Barbero, J.; Mootoo, D. R. Org. Lett. 2007, 9 , 1441.

(8) Linek, K.; Alfoldi, J.; Defaye, J. Carbohydr. Res. 1993, 247, 329.

(9) Jarreton, O.; Skrydstrup, T.; Espinosa, J.-F.; Jimenez-Barbero, J.; Beau, J.-M. Chem. Eur. J. 1999, 5, 430 .

(10) Micheel, F.; Heinemann, K. H.; Schwieger, K. H.; Frowein, A.Tetrahedron Lett. 1965, 42, 3769.

(11) Wong, C.-H.; Moris-Varas, F.; Hung, S.-C.; Marron, T. G.; Lin, C.-C.; Gong, K. W.; WeitzSchmidt, G. J. Am. Chem. Soc. 1997, 119, 8152. 
(12) Postema, M. H. D.; Piper, J. L.; Liu, L.; Shen, J.; Faust, M.; Andreana, P. J. Org. Chem. 2003, $68,4748$.

(13) Waldscheck, B.; Streiff, M.; Notz, W.; Kinzy, W.; Schmidt, R. R. Angew. Chem. Int. Ed. 2001, $40,4007$.

(14) Freudenberg, K.; Oertzen, K. Liebigs Ann. Chem.1951, 574, 37.

(15) Kawai, Y.; Ando, H.; Ozeki, H.; Koketsu, M.; Ishihara, H. Org. Lett. 2005, 7, 4653.

(16) Zeitouni, J.; Norsikian, S.; Merlet, D.; Lubineau, A. Adv. Synth. Catal. 2006, 348, 1662.

(17) Renaudet, O.; Dumy, P. Tetrahedron Lett. 2002, 58, 2127.

(18) Chakka, N.; Johnston, B. D.; Pinto, B. M. Can. J. Chem. 2005, 83, 929.

(19) Miquel, N.; Doisneau, G. ; Beau, J.-M. Angew. Chem. Int. Ed. 2000, 39, 4111.

(20) Peri, F.; Deutman, A.; La Ferla, B.; Nicotra, F. Chem. Commun. 2002, 1504.

(21) Fiore, M.; Marra, A.; Dondoni, A. J. Org. Chem. 2009, 74, 4422.

(22) Czernecki, S.; Randriamandimby, D. J. Carbohydr. Chem. 1996, 15, 183.

(23) Maijs, L.; Fisers, G.; Tsereteli, I. Y. Latvijas PSR Zinatnu Akademijas Vestis, Kimijas Serija 1969, 4, 496.

(24) Lopez, M.; Drillaud, N.; Bornaghi, L. F.; Poulsen, S.-A. J. Org. Chem. 2009, 74, 2811.

(25) Lopez, M.; Bornaghi, L. F.; Innocenti, A.; Vullo, D.; Charman, S. A.; Supuran, C. T.; Poulsen, S.-A. J. Med. Chem. 2010, 53, 2913.

(26) Pfrengle, F. Reissig, H. -U. Chem. Soc. Rev. 2010, 39, 549.

(27) Ibatullin, F. M.; Shabalin, K. A.; Jänis, J. V.; Shavvac, A. G. Tetrahedron Lett. 2003, 44, 7961. (28) Zemplén, G. Ber. Dtsch. Chem. Ges .1926, 59, 1254.

(29) Bell, R. H.; Horton, D. Carbohydr. Res. 1969, 9, 187.

(30) Linek, K.; Alfödi, J.; Defaye, J. Carbohydr. Res. 1987, 195.

(31) Moitessier, N. ; Chapleur, Y. Tetrahedron Lett. 2003, 44, 1731. 
(32) Smith, D. A.; Jones, R. M. Curr. Opin. Drug Disc. Dev. 2008, 72.

(33) Perrin, C. L.; Armstrong, K. B. J. Am. Chem. Soc. 1993, 115, 6825.

(34) Guo, J.; Frost, J. W. J. Am. Chem. Soc. 2002, 124, 10642.

(35) Reist, E. J.; Spencer, R. R.; Calkins, D. F.; Baker, B. R.; Goodman, L. J. Org. Chem. 1965, 30, 2312.

(36) Ramirez, J.; Yu, L.; Li, J.; Braunschweiger, P. G.; Wang, P. G. Bioorg. Med. Chem. Lett. 1996, $6,2575$.

(37) Christiansen-Brams, I.; Meldal, M.; Bock, K. J. Chem. Soc., Perkin Trans. 1 1993, 1461.

(38) Baer, H. H.; Gan, Y. Carbohydr. Res. 1991, 210, 233.

(39) Tronchet, J. M. J.; Poncet, J. Carbohydr. Res. 1975, 42, 347. 